October 22, 2018

\title{
Generalized Geometry in AdS/CFT and Volume Minimization
}

\author{
Maxime Gabella ${ }^{1}$ and James Sparks ${ }^{2}$ \\ 1: Rudolf Peierls Centre for Theoretical Physics, \\ University of Oxford, \\ 1 Keble Road, Oxford OX1 3NP, U.K. \\ 2: Mathematical Institute, University of Oxford, \\ 24-29 St Giles', Oxford OX1 3LB, U.K.
}

\begin{abstract}
We study the general structure of the $A d S_{5} / \mathrm{CFT}_{4}$ correspondence in type IIB string theory from the perspective of generalized geometry. We begin by defining a notion of "generalized Sasakian geometry," which consists of a contact structure together with a differential system for three symplectic forms on the four-dimensional transverse space to the Reeb vector field. A generalized Sasakian manifold which satisfies an additional "Einstein" condition provides a general supersymmetric $A d S_{5}$ solution of type IIB supergravity with fluxes. We then show that the supergravity action restricted to a space of generalized Sasakian structures is simply the contact volume, and that its minimization determines the Reeb vector field for such a solution. We conjecture that this contact volume is equal to the inverse of the trial central charge whose maximization determines the R-symmetry of any four-dimensional $\mathcal{N}=1$ superconformal field theory. This variational procedure allows us to compute the contact volumes for a predicted infinite family of solutions, and we find perfect agreement with the central charges and R-charges of BPS operators in the dual mass-deformed generalized conifold theories.
\end{abstract}




\section{Contents}

1 Introduction and results 2

2 Generalized Sasakian geometry $\quad 6$

2.1 Aspects of generalized geometry . . . . . . . . . . . . 6 6

2.2 Generalized Calabi-Yau structure . . . . . . . . . . . . 10

2.3 Compatible structure of symplectic type . . . . . . . . . . . 15

2.4 Differential system . . . . . . . . . . . . . . . . . . 23

2.4.1 Reduction .......................... 23

2.4 .2 Einstein condition . . . . . . . . . . . . 25

2.4 .3 Symplectic triple . . . . . . . . . . . . . . 26

2.5 Example: $\beta$-transform of Kähler cones . . . . . . . . . . . . . . . 28

3 Volume minimization $\quad 30$

3.1 Supergravity action . . . . . . . . . . . . . . . . 30

3.1.1 A five-dimensional action . . . . . . . . . . . . . . 31

3.1.2 On-shell action and central charge . . . . . . . . . . . . 33

3.2 Restriction to generalized Sasakian manifolds . . . . . . . . . . . . 34

3.3 Volume minimization: summary . . . . . . . . . . . . . 39

3.4 Relation to $a$-maximization . . . . . . . . . . . . . 42

4 Massive deformation of generalized conifolds

4.1 Massive deformation of $\mathcal{N}=4$ super-Yang-Mills theory . . . . . . . . 45

4.2 Suspended pinch point . . . . . . . . . . . . . . 48

4.3 Generalized conifolds . . . . . . . . . . . . . . 56

5 Conclusion and outlook 59

A The hazards of dimensional reduction

\begin{tabular}{l|l} 
B The contact volume functional & 62
\end{tabular} 


\section{Introduction and results}

One of the most significant advances in contemporary theoretical physics has been the realization that certain geometric backgrounds in string theory have completely equivalent descriptions as ordinary quantum field theories. Thanks to this gauge/gravity correspondence, many new insights have been obtained on both sides. The archetypal example is the $A d S_{5} \times S^{5}$ solution of type IIB supergravity, with the round Einstein metric on $S^{5}$, which corresponds to $\mathcal{N}=4$ super-Yang-Mills theory [1]. In fact, according to the AdS/CFT correspondence, any supersymmetric $A d S_{5}$ solution admits a dual description in terms of a four-dimensional superconformal field theory (SCFT). In this paper we shall elucidate the geometric structure of such general solutions, and explain how it maps to important properties of SCFTs.

Following on from the $A d S_{5} \times S^{5}$ solution, a rich class of special solutions takes the form $A d S_{5} \times Y_{\mathrm{SE}}$, where $Y_{\mathrm{SE}}$ is a Sasaki-Einstein five-manifold [2, 5, 3, 4]. The latter is by definition a compact five-manifold whose metric cone $C\left(Y_{\mathrm{SE}}\right)$ is Kähler and Ricci-flat, i.e. Calabi-Yau. The dual SCFTs have $\mathcal{N}=1$ supersymmetry and can be understood as arising on a stack of D3-branes located at the apex of the cone. Some essential properties of these SCFTs, such as the central charge and the conformal dimensions of chiral primary operators, are captured by their Abelian R-symmetry [6]. This corresponds geometrically to a canonical Killing vector field $\xi$, which is real holomorphic with respect to the complex structure on the Calabi-Yau cone, and is also the Reeb vector field associated with the contact structure on $Y_{\mathrm{SE}}$ descending from the symplectic structure of the cone. Recall here that a contact form on an odd-dimensional manifold of dimension $2 n-1$ is a one-form $\sigma$ such that $\sigma \wedge(\mathrm{d} \sigma)^{n-1}$ is nowhere zero, i.e. a volume form. It is a standard result that there is always a unique vector field $\xi$, called the Reeb vector field, such that $\xi\lrcorner \sigma=1, \xi\lrcorner \mathrm{d} \sigma=0$. The central charge of the field theory may then be expressed as the volume of $Y_{\mathrm{SE}}$ [7, 8], while the volumes of supersymmetric three-submanifolds give the conformal dimensions of chiral primary operators corresponding to wrapped D3-branes.

The most general solutions of type IIB supergravity with fluxes dual to $\mathcal{N}=1$ SCFTs take the form $A d S_{5} \times Y$, where $Y$ is a compact Riemannian five-manifold that in general is not Sasaki-Einstein. The requirement of supersymmetry puts certain constraints on $Y$, and in particular the cone $C(Y)$ is generalized Calabi-Yau [9], in the sense of Hitchin [10] that it carries a non-degenerate closed pure spinor, and thus an integrable generalized complex structure. In addition to this, there is a second 
compatible generalized complex structure, which although non-integrable nevertheless implies that the cone is symplectic [9] (provided the five-form flux sourced by D3-branes is non-vanishing). Precisely as in the Sasaki-Einstein case, one can define a canonical Killing vector field which is also the Reeb vector field $\xi$ associated with the induced contact structure on $Y$. The difference with the Sasaki-Einstein case is that $\xi$ is now generalized holomorphic, that is holomorphic with respect to the generalized complex structure (there is no complex structure in general). Remarkably, the volume formulas for the central charge and the conformal dimensions of chiral primary operators still hold in this generalized setting, but now in terms of contact volumes [11].1]

As shown in [8, 12, a very useful perspective is to regard Sasaki-Einstein metrics as critical points of the Einstein-Hilbert action restricted to a space of Sasakian metrics, whose cones are by definition Kähler but not necessarily Ricci-flat. More precisely, on the space of Sasakian metrics whose cones admit a nowhere-vanishing holomorphic $(3,0)$-form $\Omega$, with homogeneous degree three under the Euler vector field $r \partial_{r}$, the Einstein-Hilbert action precisely reduces to the volume functional. This volume actually depends only on the Reeb vector field, and a critical point hence determines the unique Reeb vector field for which a Sasakian manifold is also Einstein, provided such a metric exists. This allows one to extract important geometric information without having to know the Sasaki-Einstein metric explicitly. This is extremely useful since there are now many existence results for Sasaki-Einstein metrics (for a review, see [13]), with vast classes of examples not known explicitly. Notwithstanding this ignorance, one can still compute the volumes of these solutions by volume minimization [8, 12] and compare them to BPS quantities in the conjectured dual SCFTs.

As first suggested in [12, the determination of the Reeb vector field by volume minimization corresponds to the determination of the R-symmetry of a four-dimensional $\mathcal{N}=1$ SCFT by $a$-maximization [14]. The procedure involves constructing a trial Rsymmetry, which mixes arbitrarily with the set of global (flavour) Abelian symmetries, and imposing anomaly cancellation constraints. The correct R-symmetry at the infrared fixed point is then the one which (locally) maximizes the trial central charge. A proof that the trial central charge function (appropriately interpreted) is equal to the inverse of the "off-shell" Sasakian volume function was presented in [15] for toric (that is, $U(1)^{3}$-invariant) Sasakian metrics, and very recently in [16] for general Sasakian metrics.

\footnotetext{
${ }^{1}$ In the Sasaki-Einstein case these contact volumes are equal to the Riemannian volumes defined by the metric. However, this is no longer be true in the general case with fluxes.
} 
Since $a$-maximization applies in principle to every $\mathcal{N}=1$ SCFT, it is clearly desirable to extend the procedure of volume minimization beyond the Sasaki-Einstein case, to the most general supersymmetric $A d S_{5}$ solutions of type IIB supergravity. This is one of the goals of the present paper. The motivation is the same as in the Sasaki-Einstein case: explicit solutions will form a very small subset of the space of all solutions, since finding them always relies on having a large amount of symmetry. On the other hand, one might hope that the development in this paper will eventually lead to existence results, say for toric $A d S_{5}$ solutions with general fluxes; then volume minimization and the results of [9, 11] will allow one to compute BPS quantities for these solutions. Following our previous work in these references, the approach we will take is to reformulate the backgrounds in terms of generalized geometry.

We begin in section 2 by expressing the constraints imposed on the cone $C(Y)$ by supersymmetry in a geometric form. After reduction on the Euler and Reeb vector fields $r \partial_{r}$ and $\xi$, we obtain a system of equations on a four-dimensional transverse space for a triple of orthogonal symplectic forms $\left(\omega_{0}, \omega_{1}, \omega_{2}\right)$, a structure first studied in [17], and two functions $h$ and $\hat{\Delta}$. More precisely, the symplectic forms satisfy

$$
\begin{aligned}
\mathrm{d} \omega_{i} & =0 \quad \forall i \in\{0,1,2\}, \\
\omega_{i} \wedge \omega_{j} & =0 \quad \forall i \neq j, \\
\omega_{0} \wedge \omega_{0} & =\alpha_{1} \omega_{1} \wedge \omega_{1}=\alpha_{2} \omega_{2} \wedge \omega_{2} \neq 0,
\end{aligned}
$$

where $\alpha_{1}$ and $\alpha_{2}$ are positive functions depending on $h$ and $\hat{\Delta}$, together with the following differential conditions:

$$
\omega_{1}=\frac{1}{2} \mathcal{L}_{\mathcal{H}_{h}} \omega_{2}, \quad \mathcal{L}_{\mathcal{H}_{h}}\left(\mathcal{L}_{\mathcal{H}_{h}} \omega_{1}\right)=\mathcal{L}_{\mathcal{H}^{-4 \hat{\Delta}}} \omega_{2}
$$

Here the notation $\left.\mathcal{H}_{h} \equiv \omega_{0}^{-1}\right\lrcorner \mathrm{d} h$ means the Hamiltonian vector field for the function $h$, with respect to the symplectic form $\omega_{0}$. This differential system defines what we will call a "generalized Sasakian geometry." 2 In fact, there is in general also a special subspace of $Y$ where $h$ diverges and $\hat{\Delta}$ is constant, along which the geometry is simply Sasakian. This "type-change locus" $\mathscr{T}$ corresponds physically to the mesonic moduli space of the dual SCFT [18, 19] - for a Sasakian manifold $\mathscr{T}$ is of course the whole space, rather than a subspace. Note that the set of conditions (1.1)-(1.4) also gives a notion of

\footnotetext{
${ }^{2}$ Note that we have defined a generalized Sasakian structure only in dimension five. Indeed, the definition is primarily motivated by the supersymmetry equations we wish to solve. It might be possible to extend the definition to manifolds of general dimension $2 n-1$, but we shall not comment further on this here.
} 
"generalized Kähler geometry" for the transverse space to the Reeb foliation, although we shall not use this terminology as the meaning is different to that already introduced in [20]. The upshot is that when such a generalized Sasakian manifold satisfies an additional condition on the lengths of the three symplectic forms, it precisely provides a general supersymmetric $A d S_{5}$ solution of type IIB supergravity (with non-zero D3brane charge). As we shall see, this additional condition is effectively the Einstein equation.

In section 3 we explain how volume minimization works for generalized Sasakian manifolds. We show that the type IIB supergravity action reduces, when restricted to a space of generalized Sasakian structures, to the contact volume, and that the latter is then a strictly convex function of the Reeb vector field, as shown in appendix B. It follows that a supersymmetric $A d S_{5}$ solution that is in the same deformation class as a given generalized Sasakian manifold is obtained by minimizing the contact volume over a space of Reeb vector fields. As a concrete example, the critical Reeb vector field for which a toric generalized Sasakian manifold satisfies also the Einstein equation is obtained by minimizing the volume of a polytope, just as in the Sasakian case.

However, in contrast to the Sasakian case, generalized volume minimization requires not the holomorphic $(3,0)$-form, but rather the pure spinor $\Omega_{-}$, which is a formal sum of one-, three-, and five-forms, to be homogeneous of degree three. This imposes additional constraints on the space of Reeb vector fields that is to be minimized over. Here, our current understanding of the space of Reeb vector fields for a deformation class of generalized Sasakian manifolds is not yet as developed as in the Sasakian case [8, 12. We will nevertheless show in examples that this space is non-trivial, and that generalized volume minimization agrees with computations in dual SCFTs. In fact, we go further and make the natural conjecture that the volume function is equal to the inverse of the trial central charge of the dual SCFT, again checking this is indeed true in examples. As a very simple illustration we recurrently refer to a so-called " $\beta$ transform" of $\mathbb{C}^{3}=C\left(S^{5}\right)$ by a bivector $\beta$, which is known to be dual to a certain marginal deformation of $\mathcal{N}=4$ super-Yang-Mills theory [21, 19, 22]. In section 4 we then study a new class of examples obtained by mass deformation of generalized conifolds $C\left(L^{m, n, m}\right)$ [23, 24]. After making some physically motivated assumptions on the geometry, we verify in this class of examples the equivalence of generalized volume minimization and $a$-maximization. 


\section{Generalized Sasakian geometry}

We begin with a brief summary of generalized geometry, focusing on the results that are most relevant for what follows. We then proceed to study a pair of compatible generalized structures with pure spinors $\Omega_{-}$and $\Omega_{+}$, on which the requirement of supersymmetry imposes the following differential constraints [25, 26]:

$$
\begin{gathered}
\mathrm{d} \Omega_{-}=0, \\
\mathrm{~d}\left(\mathrm{e}^{-A} \operatorname{Re} \Omega_{+}\right)=0, \quad \mathrm{dd}^{\mathcal{J}_{-}}\left(\mathrm{e}^{-3 A} \operatorname{Im} \Omega_{+}\right)=0 .
\end{gathered}
$$

Here the function $A$ is a conformal factor, $\mathcal{J}_{-}$is the (integrable) generalized complex structure associated with $\Omega_{-}$, and $\mathrm{d}^{\mathcal{J}_{-}} \equiv\left[\mathcal{J}_{-}\right.$, d]. If $\Omega_{-}$and $\Omega_{+}$have the same length, with respect to the Mukai norm, then with the addition of an appropriate conical symmetry these conditions are equivalent to those for a supersymmetric $A d S_{5}$ solution of type IIB supergravity [9]. However, relaxing this compatibility condition on the lengths of $\Omega_{ \pm}$will give us our definition of a generalized Sasakian manifold; in particular, this definition reduces to the definition of a Sasakian manifold 3 in the case without fluxes. The closure of $\Omega_{-}$implies that the cone is generalized Calabi-Yau, in the sense of Hitchin [10]. The generalized Darboux theorem of [20] then allows one to locally put $\Omega_{-}$into a normal form. The second generalized structure $\Omega_{+}$is instead related to the background fluxes. This structure is not integrable, but it nevertheless provides a symplectic structure on the cone. After reduction along the Euler and Reeb vector fields, the compatibility condition between $\Omega_{-}$and $\Omega_{+}$leads to a system involving a symplectic triple on the transverse space to the Reeb foliation. More precisely, this is true away from a sublocus along which the generalized Sasakian structure in fact becomes Sasakian. The fluxes also satisfy a Bianchi identity, the third equation in (2.1), which gives an additional differential constraint.

\section{$2.1 \quad$ Aspects of generalized geometry}

We first recall a few relevant facts about generalized complex geometry. We refer the reader to section 2 of [9] for a concise review, to [20] for an extensive mathematical introduction, or to the review [27] for physicists.

A pure spinor $\Omega$ on a manifold $X$, in the generalized geometry sense, can be understood as a formal sum of complex differential forms of even or odd degrees, on which

\footnotetext{
${ }^{3}$ Strictly speaking this gives a Sasakian manifold which is transversely Fano, as defined for example in [13.
} 
the Clifford algebra action of a generalized vector field $V=v+\nu \in \Gamma\left(T X \oplus T^{*} X\right)$ is given by $V \cdot \Omega \equiv v\lrcorner \Omega+\nu \wedge \Omega$. More precisely, the even and odd forms of indefinite degree on a $d$-dimensional manifold $X$ are irreducible representations of the Clifford algebra $\operatorname{Cliff}(d, d)$ associated with $T X \oplus T^{*} X$, with even and odd chirality respectively. The annihilator space of $\Omega$ is defined as $L_{\Omega} \equiv\left\{V \in \Gamma\left(\left(T X \oplus T^{*} X\right) \otimes \mathbb{C}\right): V \cdot \Omega=0\right\}$, and then purity of $\Omega$ means that $L_{\Omega}$ is maximal isotropic, i.e. as a vector subbundle it has real rank $2 d$.

We shall also require $\Omega$ to be non-degenerate, in the sense that its Mukai pairing $\langle\Omega, \bar{\Omega}\rangle$ is nowhere zero on $X$. Here the bar denotes complex conjugation, and the Mukai pairing of two generalized spinors $\Phi$ and $\Psi$, or equivalently indefinite degree complex differential forms, is the top-form $\left.\langle\Phi, \Psi\rangle \equiv[\Phi \wedge \lambda(\Psi)]\right|_{\text {top }}$, where $\lambda\left(\Psi_{p}\right) \equiv(-1)^{[p / 2]} \Psi_{p}$ for $\Psi_{p}$ a $p$-form, and $[p / 2]$ denotes the integer part of $p / 2$. A non-degenerate pure spinor $\Omega$ then has an associated generalized almost complex structure $\mathcal{J}$. This is an endomorphism of $T X \oplus T^{*} X$ with $\mathcal{J}^{2}=-1$, which is defined by identifying the $+\mathrm{i}$ eigenspace of $\mathcal{J}$ with $L_{\Omega}$. The condition $\mathrm{d} \Omega=V \cdot \Omega$, for some generalized vector field $V$, is equivalent to $\mathcal{J}$ being integrable. This means that $L_{\Omega}$ is closed under the Courant bracket [10]. If $X$ is equipped with a non-degenerate closed pure spinor, $\mathrm{d} \Omega=0$, then $X$ is called generalized Calabi-Yau in the sense of Hitchin [10]. 4 ]

At any given point on $X$ a pure spinor takes the general form

$$
\Omega=\theta_{1} \wedge \cdots \wedge \theta_{k} \wedge \mathrm{e}^{-b+\mathrm{i} \omega}
$$

where the $\theta_{i}$ are complex one-forms and $b, \omega$ real two-forms. The integer $k$ is called the type of the pure spinor, at that point. For example, the holomorphic $(3,0)$-form on a Calabi-Yau three-fold is a pure spinor that is everywhere of type three, with $b=\omega=0$. On the other hand, a symplectic form $\omega$ gives rise to a pure spinor $\mathrm{e}^{\mathrm{i} \omega}$ that is everywhere of type zero. In this paper we will want to replace the holomorphic $(3,0)$-form on a Calabi-Yau three-fold by a pure spinor of type one on a dense open subset of $X$, but which perhaps changes to type three along a special sublocus - the "type-change locus".

We will be interested in manifolds with a pair of compatible generalized structures $\mathcal{J}_{-}$ and $\mathcal{J}_{+}$. The compatibility condition means that the structures commute, $\left[\mathcal{J}_{-}, \mathcal{J}_{+}\right]=0$, which can equivalently be expressed as $\mathcal{J}_{-} \cdot \Omega_{+}=0$, where the induced action of $\mathcal{J}_{-}$ is via the Lie algebra action, or as $\left\langle\Omega_{-}, V \cdot \Omega_{+}\right\rangle=0=\left\langle\bar{\Omega}_{-}, V \cdot \Omega_{+}\right\rangle$for all generalized

\footnotetext{
${ }^{4}$ Beware the existence of a different definition of generalized Calabi-Yau in 20] which requires two (compatible) integrable generalized structures.
} 
vectors $V$. One also requires that $\mathcal{J}_{-}$and $\mathcal{J}_{+}$define a generalized metric through

$$
G \equiv-\mathcal{J}_{-} \mathcal{J}_{+}
$$

which encapsulates an ordinary Riemannian metric $g$ and a real two-form $B$ :

$$
G=\left(\begin{array}{cc}
g^{-1} B & g^{-1} \\
g-B g^{-1} B & -B g^{-1}
\end{array}\right)=\left(\begin{array}{cc}
1 & 0 \\
-B & 1
\end{array}\right)\left(\begin{array}{cc}
0 & g^{-1} \\
g & 0
\end{array}\right)\left(\begin{array}{cc}
1 & 0 \\
B & 1
\end{array}\right) .
$$

Our conventions are that the $B$-transform by a two-form $B$ and the $\beta$-transform by a bivector $\beta$ act on a generalized vector $V=v+\nu$ as

$$
\left.\mathrm{e}^{B} V=\left(\begin{array}{cc}
1 & 0 \\
B & 1
\end{array}\right)\left(\begin{array}{l}
v \\
\nu
\end{array}\right)=v+(\nu-v\lrcorner B\right),
$$

and

$$
\left.\mathrm{e}^{\beta} V=\left(\begin{array}{cc}
1 & \beta \\
0 & 1
\end{array}\right)\left(\begin{array}{l}
v \\
\nu
\end{array}\right)=(v+\beta\lrcorner \nu\right)+\nu,
$$

respectively. The spinorial actions on a pure spinor $\Omega$ are then $\mathrm{e}^{B} \Omega=\left(1+B+\frac{1}{2} B \wedge\right.$ $B+\cdots) \wedge \Omega$ and $\left.\left.\mathrm{e}^{\beta} \Omega=\left(1+\beta+\frac{1}{2} \beta\right\lrcorner \beta+\cdots\right)\right\lrcorner \Omega$.

Finally, the Riemannian metric $g$ obtained via (2.3) and (2.4) allows us to define the Mukai norm of $\Omega$ (specializing now to $d=6$ ) via

$$
\|\Omega\|^{2} \equiv \mathrm{i}\langle\Omega, \bar{\Omega}\rangle / \operatorname{vol}_{X},
$$

where $\operatorname{vol}_{X}$ denotes the Riemannian volume form of the metric $g$. Similarly, $g$ defines a Hodge star operator $\star$, through which we may define the following norms:

$$
|\Omega|^{2} \equiv\langle\Omega, \star \lambda(\bar{\Omega})\rangle / \operatorname{vol}_{X}, \quad|\Omega|_{B}^{2} \equiv\left|\mathrm{e}^{B} \Omega\right|^{2} .
$$

\section{Example: the generalized structure of Calabi-Yau cones}

As a simple example, let us apply the above language to the familiar case of CalabiYau three-folds. Here $X$ is equipped with a pair of compatible pure spinors $\Omega_{-}$and $\Omega_{+}$given by

$$
\begin{aligned}
& \Omega_{-}=\bar{\Omega}, \\
& \Omega_{+}=\exp (\mathrm{i} \omega),
\end{aligned}
$$


where $\Omega$ is a holomorphic (3,0)-form and $\omega$ is the Kähler form 5 For example, taking $X=\mathbb{C}^{3}$, equipped with its flat metric, we have $\Omega=\mathrm{d} z_{1} \wedge \mathrm{d} z_{2} \wedge \mathrm{d} z_{3}$ and corresponding Kähler form $\omega=\frac{\mathrm{i}}{2} \sum_{i=1}^{3} \mathrm{~d} z_{i} \wedge \mathrm{d} \bar{z}_{i}$, where $z_{1}, z_{2}, z_{3}$ are standard complex coordinates on $\mathbb{C}^{3}$.

In this case both pure spinors are closed, $\mathrm{d} \Omega_{-}=\mathrm{d} \Omega_{+}=0$, and thus the corresponding generalized almost complex structures are integrable. These are

$$
\mathcal{J}_{-}=\left(\begin{array}{cc}
I & 0 \\
0 & -I^{*}
\end{array}\right), \quad \mathcal{J}_{+}=\left(\begin{array}{cc}
0 & \omega^{-1} \\
-\omega & 0
\end{array}\right),
$$

respectively, where $I$ denotes the integrable complex structure tensor on $X$ and $\omega$ is the Kähler (hence compatible and symplectic) form. Indeed, the compatibility condition gives $I^{*} \cdot \omega=0$, which says that $\omega$ is a $(1,1)$-form with respect to the complex structure I. From the expressions for $\mathcal{J}_{-}$and $\mathcal{J}_{+}$above, we obtain from (2.3) that

$$
g=\omega(I, \cdot), \quad B=0
$$

Finally, for a Ricci-flat Kähler metric, the Mukai pairings of the pure spinors are equal:

$$
\frac{\mathrm{i}}{8}\left\langle\Omega_{-}, \bar{\Omega}_{-}\right\rangle=\frac{\mathrm{i}}{8} \Omega \wedge \bar{\Omega}=\frac{1}{3 !} \omega^{3}=\frac{\mathrm{i}}{8}\left\langle\Omega_{+}, \bar{\Omega}_{+}\right\rangle .
$$

Without this condition one instead has only a Kähler metric on a complex manifold with zero first Chern class.

For application to $A d S_{5}$ solutions of string theory, we are interested more specifically in conical geometries, as explained in detail in [9]. In the above context of CalabiYau solutions, this means that by definition the Kähler metric takes the conical form $g=\mathrm{d} r^{2}+r^{2} g_{Y}$, with $g_{Y}$ a metric on some compact five-manifold base $Y$, and that the holomorphic $(3,0)$-form $\Omega$ is homogeneous of degree three under the Euler vector field $r \partial_{r}$. Such geometries solve $\mathrm{d} \Omega_{-}=\mathrm{d} \Omega_{+}=0$ with the definitions (2.9), but not necessarily the equal norm condition (2.12); this implies that $g_{Y}$ is a Sasakian metric. In fact, this is a slightly special Sasakian structure, since the general definition requires only that the cone is complex, not that its first Chern class vanishes. Notice, however, that $\Omega_{+}$defined by (2.9) does not have a well-defined scaling dimension, since the Kähler form $\omega$ has scaling dimension two. Instead, the pure spinors associated with the Calabi-Yau cones appearing in the AdS/CFT correspondence take the rescaled

${ }^{5}$ The complex conjugation in $\Omega_{-}=\bar{\Omega}$ is due to an unfortunate choice of conventions in the generalized geometry literature; an explanation may be found in [9], pages 10 and 11. 
forms [9]:

$$
\begin{aligned}
& \Omega_{-}=\bar{\Omega}, \\
& \Omega_{+}=-\mathrm{i} r^{3} \exp \left(\frac{\mathrm{i}}{r^{2}} \omega\right) .
\end{aligned}
$$

This comes about because the ten-dimensional solutions of interest have the form $A d S_{5} \times Y$, which can also be viewed using Poincaré coordinates as the warped product $\mathbb{R}^{1,3} \times X$ where $X=C(Y) \cong \mathbb{R}_{+} \times Y$ and $r$ is a coordinate on $\mathbb{R}_{+}$. Both pure spinors in (2.13) are now homogeneous of degree three, and the resulting Riemiannian metric on $X=C(Y)$ is conformal to the cone metric $g$ above: $g_{X}=r^{-2}\left(\mathrm{~d} r^{2}+r^{2} g_{Y}\right)$. Notice that $g_{X}$ here is homogeneous of degree zero, and in fact setting $t=\log r \in(-\infty, \infty)$ we see that $g_{X}$ is a cylinder over $Y: g_{X}=\mathrm{d} t^{2}+g_{Y}$. The crucial difference after the rescaling is that $\Omega_{+}$is no longer closed, and hence $\mathcal{J}_{+}$is not integrable. This may sound peculiar, but these are the natural pure spinors associated with the geometry in AdS/CFT. Indeed, the lack of closure of $\Omega_{+}$may be understood as due to the presence of background fluxes on the cone. In the Sasaki-Einstein case discussed in this example,

only the five-form flux $F_{5}$ is non-zero, and it is the presence of this non-zero D3-brane flux that obstructs the integrability of $\mathcal{J}_{+}$.

In this paper we will study the generalization of the above geometric structure to the case where all the background fields of type IIB supergravity, including the $B$-field and all the components of the Ramond-Ramond (RR) fluxes, are turned on.

\subsection{Generalized Calabi-Yau structure}

In this section we consider an integrable generalized complex structure $\mathcal{J}_{-}$on a sixmanifold $X$ that is associated with a closed pure spinor $\Omega_{-}$:

$$
\mathrm{d} \Omega_{-}=0 \text {. }
$$

According to Hitchin's definition [10], this makes $X$ a generalized Calabi-Yau manifold. We will also choose $\Omega_{-}$to be of odd type, such that over a dense open subset $X_{0} \subset X$ it has type one. This is the lowest odd type possible, but at special loci the type may change to type three. As explained in [9], this is the case of interest for application to $A d S_{5}$ solutions of type IIB string theory. We note that more generally $\Omega_{-}$will always have a least type $k$ on $X$, taken over all points in $X$, and that the subset of $X$ where $\Omega_{-}$ has type $k$ is then dense. For example, as already mentioned, a Calabi-Yau three-fold is everywhere of type $k=3$. 
In the remainder of this section we focus almost exclusively on the dense open set $X_{0} \subset X$ where $\Omega_{-}$has type one. The limit points of $X_{0}$ are then by assumption type three, and one can view these as imposing certain boundary conditions on the various type one objects on $X_{0}$ that we study. In fact we shall not study these boundary conditions in detail here, since for our purposes it will be sufficient to know simply the local conditions on $X_{0}$, together with the fact that certain structures are in fact defined globally on $X$.

The most general algebraic form for a closed polyform of type $k=1$ is [20]

$$
\theta \wedge \mathrm{e}^{-b_{-}+\mathrm{i} \omega_{-}}
$$

with $\theta$ a complex one-form and $b_{-}, \omega_{-}$real two-forms. By the generalized Darboux theorem [20], this structure is locally equivalent, via a diffeomorphism and a closed $B$ transform (2.5), to the direct sum of a complex structure of complex dimension one and a symplectic structure of real dimension four. More precisely, for any point in $X_{0}$ there is a neighbourhood with a symplectic foliation that is isomorphic to an open set in $\mathbb{C} \times$ $\mathbb{R}^{4}$, with transverse complex coordinate $z=x+\mathrm{i} y$ and real coordinates $\left\{x_{1}, y_{1}, x_{2}, y_{2}\right\}$ on the symplectic leaves 6 The appropriate leaf-preserving diffeomorphism $\varphi$ is such that the pull-back of the two-form $\omega_{-}$to each leaf is the standard Darboux symplectic form $\omega_{0}$ :

$$
\left.\varphi^{*} \omega_{-}\right|_{\mathbb{R}^{4} \times\{\mathrm{pt}\}}=\omega_{0} \equiv \mathrm{d} x_{1} \wedge \mathrm{d} y_{1}+\mathrm{d} x_{2} \wedge \mathrm{d} y_{2}
$$

The freedom to shift the exponent in (2.15) by a two-form whose wedge product with $\theta$ vanishes allows one to trade $b_{-}$for a closed two-form $b_{0}$, and obtain 7

$$
\varphi^{*}\left[\theta \wedge \exp \left(-b_{-}+\mathrm{i} \omega_{-}\right)\right]=\mathrm{d} \bar{z} \wedge \mathrm{e}^{-b_{0}+\mathrm{i} \omega_{0}} .
$$

We dispose of $b_{0}$ by a closed $B$-transform, and take the resulting polyform as the definition of $\Omega_{-}$in this open neighbourhood:

$$
\Omega_{-} \equiv \mathrm{d} \bar{z} \wedge \mathrm{e}^{\mathrm{i} \omega_{0}}
$$

In the application to physics, the above closed $B$-transform will also act on the compatible pure spinor $\Omega_{+}$introduced in section 2.3 , and will be reabsorbed into its definition.

\footnotetext{
${ }^{6}$ When we introduce the compatible pure spinor $\Omega_{+}$later, we shall see that there is another foliation by orbits of $\partial_{z}$. In fact the latter will turn out to be a global vector field on $X$, not simply a local vector field in a neighbourhood of a point in $X_{0}$, and on $Y$ this will reduce to a Reeb foliation (see subsection 2.4.1).

7 The reason for choosing the anti-holomorphic one-form $\mathrm{d} \bar{z}$ is to align with the sign conventions in [9], cf. footnote 1 there, and footnote 5 here.
} 
Notice that such closed $B$-transforms are symmetries of the supergravity equations, but that globally only integer period closed $B$-transforms are symmetries of string theory.

The generalized structure corresponding to (2.18) combines a standard complex structure $I_{0}$ on the complex leaf space with a symplectic structure on the leaves (recall the standard examples in (2.10) $)$. In the coordinate basis $\left\{\partial_{z}, \partial_{\bar{z}}, \partial_{x_{1}}, \partial_{y_{1}}, \cdots, \mathrm{d} x_{2}, \mathrm{~d} y_{2}\right\}$ of $\left(T X \oplus T^{*} X\right) \otimes \mathbb{C}$ it is given by

$$
\mathcal{J}_{-}=\left(\begin{array}{cc|cc}
I_{0} & & 0_{2} & \\
& 0_{4} & & \omega_{0}^{-1} \\
\hline 0_{2} & & -I_{0}^{*} & \\
& -\omega_{0} & & 0_{4}
\end{array}\right) .
$$

The two-form $\omega_{0}$ gives an isomorphism between the tangent and cotangent spaces of the leaves, as $\omega_{0}:\left(\partial_{x_{a}}, \partial_{y_{a}}\right) \mapsto\left(\mathrm{d} y_{a},-\mathrm{d} x_{a}\right), a=1,2$, with inverse $\omega_{0}^{-1}:\left(\mathrm{d} x_{a}, \mathrm{~d} y_{a}\right) \mapsto$ $\left(-\partial_{y_{a}}, \partial_{x_{a}}\right)$. The group action of $\mathcal{J}_{-}$, viewed as an element of $O(d, d)$, is

$$
\begin{aligned}
& \mathcal{J}_{-}\left(\partial_{z}\right)=\mathrm{i} \partial_{z}, \quad \mathcal{J}_{-}\left(\partial_{\bar{z}}\right)=-\mathrm{i} \partial_{\bar{z}}, \\
& \mathcal{J}_{-}(\mathrm{d} z)=-\mathrm{id} z, \quad \mathcal{J}_{-}(\mathrm{d} \bar{z})=\mathrm{id} \bar{z}, \\
& \mathcal{J}_{-}\left(\partial_{x_{a}}\right)=\mathrm{d} y_{a}, \quad \mathcal{J}_{-}\left(\partial_{y_{a}}\right)=-\mathrm{d} x_{a}, \\
& \mathcal{J}_{-}\left(\mathrm{d} x_{a}\right)=\partial_{y_{a}}, \quad \mathcal{J}_{-}\left(\mathrm{d} y_{a}\right)=-\partial_{x_{a}} \text {. }
\end{aligned}
$$

On the other hand, $\mathcal{J}_{-}$may also be regarded as an element of the Lie algebra $o(d, d)$, and the algebra action of $\mathcal{J}_{-}$on differential forms is then defined via the Clifford action as $\left.\mathcal{J}_{-} \cdot \equiv-I_{0}^{*} \cdot-\omega_{0} \wedge+\omega_{0}^{-1}\right\lrcorner$, with the bivector $\omega_{0}^{-1} \equiv \partial_{y_{1}} \wedge \partial_{x_{1}}+\partial_{y_{2}} \wedge \partial_{x_{2}}$.

\section{Example: $\beta$-transform of $\mathbb{C}^{3}$}

Let $\left\{z_{1}, z_{2}, z_{3}\right\}$ be standard complex coordinates on $\mathbb{C}^{3}$, which is the complex structure associated with the pure spinor

$$
\Omega=\mathrm{d} z_{1} \wedge \mathrm{d} z_{2} \wedge \mathrm{d} z_{3} .
$$


If we deform as in (2.6) by a bivector 8

$$
\beta=z_{1} \partial_{z_{2}} \wedge z_{2} \partial_{z_{1}}+\text { c.p. }
$$

where "c.p." means the cyclic permutations of pairs of indices $\{1,2,3\}$, we obtain

$$
\begin{aligned}
\mathrm{e}^{\beta} \Omega & =\mathrm{d}\left(z_{1} z_{2} z_{3}\right)+\mathrm{d} z_{1} \wedge \mathrm{d} z_{2} \wedge \mathrm{d} z_{3} \\
& =\mathrm{d}\left(z_{1} z_{2} z_{3}\right) \wedge \exp \left(\frac{\mathrm{d} z_{1} \wedge \mathrm{d} z_{2}}{3 z_{1} z_{2}}+\text { c.p. }\right)
\end{aligned}
$$

This deformed pure spinor is of type three on the locus $\left\{\mathrm{d}\left(z_{1} z_{2} z_{3}\right)=0\right\}$, corresponding to the union of the three complex lines $\left\{z_{i}=z_{j}=0 \mid i, j=1,2,3, i<j\right\}$, but is otherwise of type one as shown by the expression on the second line.

We now assume that $X \cong \mathbb{R}_{+} \times Y$, with $Y$ compact, and introduce an appropriate homogeneity property under $r \partial_{r}$, where $r$ is a coordinate on $\mathbb{R}_{+}$. For a Calabi-Yau cone, recall that the holomorphic $(3,0)$-form is required to be homogeneous of degree three under $r \partial_{r}$; that is, $\mathcal{L}_{r \partial_{r}} \Omega=3 \Omega$. Following [9], we thus similarly impose this condition on the polyform $\Omega_{-}$:

$$
\mathcal{L}_{r \partial_{r}} \Omega_{-}=3 \Omega_{-}
$$

This gives in general separate conditions on each of the one-, three-, and five-form components of $\Omega_{-}$. Note that this implies that $r \partial_{r}$ is generalized holomorphic, $\mathcal{L}_{r \partial_{r}} \mathcal{J}_{-}=$ 0. Again following [9], we define the generalized Reeb vector $\xi=\xi_{\mathrm{v}}+\xi_{\mathrm{f}} \in \Gamma\left(T X \oplus T^{*} X\right)$ and the generalized contact form $\eta=\eta_{\mathrm{v}}+\eta_{\mathrm{f}} \in \Gamma\left(T X \oplus T^{*} X\right)$ as

$$
\xi \equiv \mathcal{J}_{-}\left(r \partial_{r}\right), \quad \eta \equiv \mathcal{J}_{-}(\mathrm{d} \log r)
$$

Here we have denoted the projections onto vector and form components by subscripts $\mathrm{v}$ and $\mathrm{f}$, respectively. From the fact that the complex combination $r \partial_{r}-\mathrm{i} \xi$ is in the

\footnotetext{
${ }^{8}$ More generally, the deformation complex of a generalized structure on a complex manifold $M$ is $\oplus_{p+q=2} H^{p}\left(M, \wedge^{q} T_{1,0}\right)$. If $M$ is a compact Calabi-Yau manifold, only $H^{1}\left(M, T_{1,0}\right)$, whose elements are ordinary complex deformations, is non-vanishing. There is therefore no bivector $\beta \in H^{0}\left(M, \wedge^{2} T_{1,0}\right)$ that can be used to deform it. However, as observed by Wijnholt [28], for Calabi-Yau manifolds $X$ that are cones over regular Sasaki-Einstein manifolds $Y$ with Kähler-Einstein base $M$, one can consider elements $\beta \in H^{0}\left(M, \wedge^{2} T_{1,0}\right)$ and then holomorphically extend these over the entire cone to obtain a non-commutative deformation. In general, there might be obstructions in $\oplus_{p+q=3} H^{p}\left(M, \wedge^{q} T_{1,0}\right)$ to the integrability of such deformations. For the $\mathbb{C P}^{2}$ base of $\mathbb{C}^{3}$, Gualtieri showed that the obstructions vanish [20].
} 
annihilator space $L_{\Omega_{-}}$, it follows that $\Omega_{-}$has a definite charge under $\xi 9$

$$
\mathcal{L}_{\xi} \Omega_{-}=-3 \mathrm{i} \Omega_{-},
$$

and so $\xi$ is generalized holomorphic as well, $\mathcal{L}_{\xi} \mathcal{J}_{-}=0$.

This homogeneity requirement leads to the following results, which for clarity we present as a proposition. Of course, these are to be understood as local expressions, defined in the coordinate patch in which $\Omega_{-}$takes the form (2.18).

\section{Proposition 1.}

a) The complex coordinate $z$ can be expressed in terms of $r$, a real function $h$, and a phase $\psi$ as:

$$
z \equiv r^{3} \mathrm{e}^{3 h} \mathrm{e}^{3 \mathrm{i} \psi}
$$

with $\partial_{r} h=\partial_{r} \psi=0$. The latter mean that $h$ and $\psi$ are pull-backs from (a neighbourhood in) $Y$. Moreover, $h$ depends only on the symplectic (leaf) coordinates.

b) The Euler vector field takes the form

$$
r \partial_{r}=3\left(z \partial_{z}+\bar{z} \partial_{\bar{z}}\right)-\mathcal{H}_{\varphi}
$$

where we define the Hamiltonian vector field $\left.\mathcal{H}_{\varphi} \equiv \omega_{0}^{-1}\right\lrcorner \mathrm{d} \varphi$ which is tangent to the symplectic leaves, and the function $\varphi$ also depends only on the symplectic coordinates.

The following proof is straightforward but somewhat technical, and may be skipped on a first reading:

\section{Proof.}

Consider the general ansatz $\mathrm{d} z=\mu_{0} \mathrm{~d} \log r+\nu_{1}$, with $\mu_{0}$ a function and $\nu_{1}$ a one-form such that $\left.r \partial_{r}\right\lrcorner \nu_{1}=0$. The one-form part of the homogeneity condition (2.23) is

$$
\mathcal{L}_{r \partial_{r}} \mathrm{~d} z=3 \mathrm{~d} z
$$

\footnotetext{
${ }^{9}$ We use the same symbol for the ordinary Lie derivative with respect to a vector field $v \in \Gamma(T X)$ and for the generalized Lie derivative with respect to a generalized vector field $V=v+\nu \in \Gamma(T X \oplus$ $\left.T^{*} X\right)$. We refer to 9 for a definition of the latter, which reduces to the ordinary Lie derivative when $\nu=0$. In particular, Cartan's formula for the action on a differential form $\Omega$ applies also in the generalized case: $\mathcal{L}_{V} \Omega=\mathrm{d}(V \cdot \Omega)+V \cdot \mathrm{d} \Omega=\mathcal{L}_{v} \Omega+\mathrm{d} \nu \wedge \Omega$.
} 
which leads to $3 \nu_{1} / \mu_{0}=\mathrm{d} \log \left(\mu_{0} / r^{3}\right)$. Since $\left.r \partial_{r}\right\lrcorner \nu_{1}=0$, we can write $\mu_{0}=3 r^{3} \mathrm{e}^{3 h} \mathrm{e}^{3 \mathrm{i} \psi}$ with $h$ and $\psi$ real functions, independent of $r$. But $\left.\mathrm{d} z=\mathrm{d}\left(r \partial_{r}\right\lrcorner \mathrm{d} z\right) / 3=\mathrm{d} \mu_{0} / 3$ and so $z=\mu_{0} / 3+c=r^{3} \mathrm{e}^{3 h} \mathrm{e}^{3 \mathrm{i} \psi}+c$, with $c$ a constant which we may set to zero by shifting the origin of $z$. This proves $a$ ), except for the last statement that $h$ is independent of $z$.

To show $b$ ), notice first that it is clear from the condition (2.28) and its complex conjugate that $r \partial_{r}$ has to contain the term $3\left(z \partial_{z}+\bar{z} \partial_{\bar{z}}\right)$. Now the three-form part of the homogeneity condition (2.23), $\mathrm{d} z \wedge \mathcal{L}_{r \partial_{r}} \omega_{0}=0$, can only be satisfied non-trivially by a term of $\mathcal{L}_{r \partial_{r}} \omega_{0}$ proportional to $\mathrm{d} z \wedge \mathrm{d} \bar{z}$. But since the action of the Lie derivative on $\omega_{0}$ will leave one symplectic component intact in every term, there is no such term in $\mathcal{L}_{r \partial_{r}} \omega_{0}$, and we must then have

$$
\mathcal{L}_{r \partial_{r}} \omega_{0}=0
$$

This fixes $r \partial_{r}$ up to a Hamiltonian vector field $\mathcal{H}_{\varphi}$ tangent to the leaves such that $\left.\mathcal{H}_{\varphi}\right\lrcorner \omega_{0}=-\tilde{\mathrm{d}} \varphi$, with $\tilde{\mathrm{d}}$ the exterior derivative along the symplectic leaves and $\varphi=$ $\varphi\left(z, \bar{z}, x_{a}, y_{a}\right)$ an arbitrary real function.

To show that $\varphi$ is independent of $z$, we use the homogeneity of $\Omega_{-}$under the generalized Reeb vector $\xi$, which in terms of generalized Darboux coordinates reads

$$
\xi=3 \mathrm{i}\left(z \partial_{z}-\bar{z} \partial_{\bar{z}}\right)+\tilde{\mathrm{d}} \varphi=\partial_{\psi}+\tilde{\mathrm{d}} \varphi
$$

This implies in particular that $\mathrm{d} \bar{z} \wedge \mathrm{d}(\tilde{\mathrm{d}} \varphi)=0$ and so $\mathrm{d}(\tilde{\mathrm{d}} \varphi)=0$, which means that locally we can write $\tilde{\mathrm{d}} \varphi=\mathrm{d} \tilde{\varphi}$, with $\tilde{\varphi}=\tilde{\varphi}\left(x_{a}, y_{a}\right)$. We can then set $\varphi=\tilde{\varphi}$.

Similarly, the generalized contact form reads

$$
\eta=\frac{\mathrm{i}}{6} \mathrm{~d} \log \frac{\bar{z}}{z}-\mathcal{H}_{h}=\mathrm{d} \psi-\mathcal{H}_{h}
$$

with the Hamiltonian vector field $\left.\mathcal{H}_{h} \equiv \omega_{0}^{-1}\right\lrcorner \mathrm{d} h$. A priori, $\eta$ contains an additional term $I_{0}^{*} \cdot \mathrm{d} h$, but the condition $\left.\mathrm{d} \bar{z} \wedge\left(\eta_{\mathrm{v}}\right\lrcorner \omega_{0}+\mathrm{d} \log r-\mathrm{i} \eta_{\mathrm{f}}\right)=0$ from the fact that $\mathrm{d} \log r-\mathrm{i} \eta$ annihilates $\Omega_{-}$gives $\mathrm{d} \bar{z} \wedge I_{0}^{*} \cdot \mathrm{d} h=0$, and so $\partial_{z} h=0$. The function $h$ is thus a function on the symplectic leaves, $h=h\left(x_{a}, y_{a}\right)$.

\subsection{Compatible structure of symplectic type}

We now introduce a second generalized structure $\mathcal{J}_{+}$on $X$, with an associated pure spinor $\Omega_{+}$. As well as being compatible with $\Omega_{-}$, we shall impose a series of conditions on this structure. Although these conditions appear directly in the supersymmetry 
analysis of [9], here we shall explain why they are natural from a purely geometric point of view, and follow carefully the consequences of each condition.

We require that $\mathcal{J}_{-}$and $\mathcal{J}_{+}$are compatible, which means that they should commute and define a positive definite generalized metric via $G \equiv-\mathcal{J}_{-} \mathcal{J}_{+}$. In the Calabi-Yau case given by (2.13),$\Omega_{+}$is everywhere type zero. We assume that this holds more generally, and thus impose that

$$
\Omega_{+} \equiv \alpha_{+} \mathrm{e}^{-b_{+}+\mathrm{i} \omega_{+}}
$$

with $\alpha_{+}$a nowhere-vanishing complex function, and $b_{+}, \omega_{+}$real two-forms. As explained in [9], in the context of string theory solutions this assumption is equivalent to the background having non-zero D3-brane charge.

The next condition we wish to impose is an appropriate homogeneity condition under the Euler vector field $r \partial_{r}$. Recall that an ordinary metric on $X=\mathbb{R}_{+} \times Y$ is said to be conical if it is homogeneous of degree two under $r \partial_{r}$, and moreover $r \partial_{r}$ is orthogonal to all tangent vectors in $Y$. Such a metric then takes the form $\mathrm{d} r^{2}+r^{2} g_{Y}$. At the end of section 2.1 we reviewed that in applying generalized geometry to $A d S_{5} \times Y$ backgrounds, the metric $g_{X}$ defined by the generalized metric (2.4) is conformal to the cone metric over $Y$, via $g_{X}=r^{-2}\left(\mathrm{~d} r^{2}+r^{2} g_{Y}\right)$. In fact this is the metric of a cylinder over $Y$, which is characterized by $\mathcal{L}_{r \partial r} g_{X}=0$ and $r \partial_{r}$ being orthogonal to the base $Y$ of the cylinder. It is then natural to extend these conditions to our generalized metric as follows:

$$
\mathcal{L}_{r \partial_{r}} G=0, \quad \text { and } \quad G\left(r \partial_{r}\right)=\mathrm{e}^{2 \hat{\Delta}} \mathrm{d} \log r
$$

with $\hat{\Delta}$ a real homogeneous function of degree zero, $\mathcal{L}_{r \partial_{r}} \hat{\Delta}=0.10$ It is straightforward to show that these conditions are equivalent to the two-form $B$ in (2.4) being basic with respect to $r \partial_{r}$, that is $\left.\mathcal{L}_{r \partial_{r}} B=r \partial_{r}\right\lrcorner B=0$, and that the metric on $X$ takes the form

$$
g_{X}=\mathrm{e}^{2 \hat{\Delta}}\left(\frac{\mathrm{d} r^{2}}{r^{2}}+g_{Y}\right),
$$

where $g_{Y}$ is the metric on the compact space $Y$. Thus $g_{X}$ is in general conformal to a cylinder metric, with $\mathrm{e}^{2 \hat{\Delta}}$ being an invariant conformal factor. The Riemannian volume form on $X$ is, with a sign convention chosen to match that of [9],

$$
\operatorname{vol}_{X} \equiv-\sqrt{g_{X}} \mathrm{~d} \log r \wedge \mathrm{d}^{5} y=-\mathrm{e}^{6 \hat{\Delta}} \mathrm{d} \log r \wedge \operatorname{vol}_{Y} .
$$

\footnotetext{
10 The function $\hat{\Delta}$ is related to the warp factor $\Delta$ and the dilaton $\phi$ in [9] through $\hat{\Delta}=\Delta+\phi / 4$. The presence of the dilaton is due to the transition from the Einstein frame $g_{\mathrm{E}}$ to the string frame $g_{\sigma}$, which is carried out by a Weyl rescaling $g_{\sigma}=\mathrm{e}^{\phi / 2} g_{\mathrm{E}}$.
} 
The already-imposed homogeneity condition $\mathcal{L}_{r \partial_{r}} G=0$ implies that $\mathcal{J}_{+}$must be invariant under $r \partial_{r}$. As for $\Omega_{-}$, we may thus similarly impose the following homogeneity condition on $\Omega_{+}$:

$$
\mathcal{L}_{r \partial_{r}} \Omega_{+}=3 \Omega_{+}
$$

From a purely geometrical point of view, it would now be natural to impose that $\mathcal{J}_{+}$ is also integrable. The manifold would then be generalized Kähler, in the sense of [20]. This is the case, for instance, in the topological string and in purely Neveu-Schwarz solutions of type II string theories - a short and highly incomplete list of references is [29, 30, 31]. For general $A d S_{5}$ solutions of type IIB string theory, however, the presence of background fluxes on the cone is an obstruction to the full integrability of $\mathcal{J}_{+}$. As we pointed out in section 2.1, this is true even for Sasaki-Einstein backgrounds. Thus integrability of $\mathcal{J}_{+}$is too strong. We instead impose the weaker differential conditions

$$
\mathrm{d}\left(\mathrm{e}^{-A} \operatorname{Re} \Omega_{+}\right)=0, \quad \mathrm{dd}^{\mathcal{J}_{-}}\left(\mathrm{e}^{-3 A} \operatorname{Im} \Omega_{+}\right)=0
$$

Here $\mathrm{e}^{A}$ is a homogeneous function of degree one, $\mathcal{L}_{r \partial_{r}} \mathrm{e}^{A}=\mathrm{e}^{A}$, and $\mathrm{d}^{\mathcal{J}_{-}} \equiv\left[\mathcal{J}_{-}, \mathrm{d}\right]$.

The first constraint in (2.37) will ensure that the cone $X$ is symplectic, as we shall prove further below. Clearly, this is a natural geometric condition to impose. The presence of the $\mathrm{e}^{-A}$ factor sets the homogeneous degree of this symplectic form to two, again as one wants for a symplectic cone.

The second constraint in (2.37) is physically none other than the Bianchi identity, $\mathrm{d}\left(\mathrm{e}^{-B} F\right)=0$, for the so-called Ramond-Ramond fluxes of type IIB supergravity. These RR fluxes can be encapsulated in the odd polyform $F \equiv F_{1}+F_{3}+F_{5}$, where $F_{p}$ is a $p$-form. From a geometric point of view, we simply define this polyform directly in terms of the imaginary part of $\Omega_{+}$as

$$
\mathrm{e}^{-B} F \equiv 8 \mathrm{~d}^{\mathcal{J}_{-}}\left(\mathrm{e}^{-3 A} \operatorname{Im} \Omega_{+}\right)
$$

Again, as part of the homogeneity conditions under $r \partial_{r}$, we impose that the RR fluxes are basic with respect to the foliation defined by $r \partial_{r}$ :

$$
\left.\mathcal{L}_{r \partial_{r}} F=0, \quad r \partial_{r}\right\lrcorner F=0
$$

These conditions now explain the presence of $\mathrm{e}^{-3 A}$ in the definition (2.38): its homogeneity property is required to balance the degree three of $\Omega_{+}$.

For the five-form component $F_{5}$, the basic condition implies that $F_{5}=f_{5} \mathrm{vol}_{Y}$, where a priori $f_{5}$ is a homogeneous function of degree zero. The final condition that we impose 
is that $f_{5}$ is a (non-zero) constant. From the string theory point of view, this follows since in type IIB supergravity the full RR five-form is self-dual, and thus of the form

$$
f_{5}\left(\operatorname{vol}_{A d S}+\operatorname{vol}_{Y}\right)
$$

The Bianchi identity is $\mathrm{d} F_{5}=H \wedge F_{3}$, but the right-hand side vanishes since by construction $H$ and $F_{3}$ are three-forms on $Y$. Thus $f_{5}$ is necessarily constant. This constant is then necessarily non-zero if $\Omega_{+}$is everywhere type zero, as we have already assumed, and as shown in Proposition 2 below.

The above are the full set of conditions we shall impose. The motivation largely came from the fact that these conditions are implied by supersymmetry, as shown in 9]. However, hopefully the above discussion also motivates these as natural geometric conditions. As we shall see later, they are not quite the full set of conditions required for a supersymmetric $A d S_{5}$ solution. In fact the structure we have now defined is in some sense the generalized version of Kähler cones, and we analogously call the base $Y$ a generalized Sasakian manifold. The following proposition summarizes the consequences of the above conditions, which also justify our use of terminology:

\section{Proposition 2.}

a) The function $\mathrm{e}^{A}$ is related to the radial function $r$ and the conformal factor $\hat{\Delta}$ through

$$
\mathrm{e}^{A}=r \mathrm{e}^{\hat{\Delta}}
$$

b) The generalized Reeb vector field $\xi$ preserves the generalized metric as well as the RR fluxes:

$$
\mathcal{L}_{\xi} G=0, \quad \mathcal{L}_{\xi}\left(\mathrm{e}^{-B} F\right)=0
$$

c) The cone is symplectic with symplectic form

$$
\omega=\frac{1}{2} \mathrm{~d}\left(r^{2} \sigma\right)
$$

where the contact one-form associated with the Reeb vector field $\xi_{\mathrm{v}}$ is

$$
\left.\left.\sigma=\eta_{\mathrm{f}}-\eta_{\mathrm{v}}\right\lrcorner b_{2}=\mathrm{d} \psi+\mathcal{H}_{h}\right\lrcorner b_{2},
$$

with $b_{2}$ a closed two-form. 
d) The pure spinor $\Omega_{+} \equiv \alpha_{+} \exp \left(-b_{+}+\mathrm{i} \omega_{+}\right)$can be expressed as

$$
\begin{aligned}
\alpha_{+} & =-\mathrm{i} \frac{f_{5}}{32} r^{3} \mathrm{e}^{-\hat{\Delta}}, \\
\omega_{+} & =\frac{\mathrm{e}^{2 \hat{\Delta}}}{r^{2}} \omega, \\
b_{+} & \left.=\mathrm{e}^{2 \hat{\Delta}} \mathrm{d} \log r \wedge \eta_{\mathrm{v}}\right\lrcorner \omega_{+}+b_{2} \\
& \left.=-\mathrm{e}^{4 \hat{\Delta}} \mathrm{d} \log r \wedge \mathcal{H}_{h}\right\lrcorner \omega_{T}+b_{2} .
\end{aligned}
$$

Here we have defined $\omega_{T} \equiv \mathrm{d} \sigma / 2$, which is the symplectic form on the transverse space to the Reeb foliation descending from $\omega$. Notice that $\Omega_{+}$being type zero implies that $f_{5} \neq 0$.

In particular, the vector part $\xi_{\mathrm{v}}$ of the generalized Reeb vector field $\xi$, defined via the integrable generalized complex structure $\mathcal{J}_{-}$in (2.24), is indeed the Reeb vector field for the contact structure induced by the symplectic form $\omega$ on the cone. Thus $Y$ is a contact manifold, and this contact structure is in some sense compatible with the generalized complex structure $\mathcal{J}_{-}$. The generalized Reeb vector field is also generalized Killing, $\mathcal{L}_{\xi} G=0$. These properties all mimick those of Kähler cones, or equivalently Sasakian manifolds. We give some examples of these generalized structures in section 2.5 below.

We now proceed to the proof of Proposition 2, This is again somewhat technical, and the reader might omit this proof on a first reading:

\section{Proof of $a$ ).}

The definition (2.38) of the RR fluxes can be rewritten as [32]

$$
\mathrm{d}\left(\mathrm{e}^{A} \operatorname{Im} \Omega_{+}\right)=\frac{1}{8} \mathrm{e}^{4 A} \mathrm{e}^{-B} \star \lambda(F),
$$

with $\lambda(F) \equiv F_{1}-F_{3}+F_{5}$. The Hodge star operator on $X$ can be written as $\star F_{p} \equiv$ $\left.(-1)^{p} F_{p}\right\lrcorner \operatorname{vol}_{X}$. Since $F_{5}=f_{5} \operatorname{vol}_{Y}$, the one-form part of (2.48) immediately gives

$$
\mathrm{d}\left(\mathrm{e}^{A} \operatorname{Im} \alpha_{+}\right)=-\frac{f_{5}}{8} \mathrm{e}^{4(A-\hat{\Delta})} \mathrm{d} \log r .
$$

Since $\mathrm{e}^{A}$ and $\mathrm{e}^{\hat{\Delta}}$ are homogeneous of degree one and zero respectively, we deduce that $\mathrm{e}^{A} \operatorname{Im} \alpha_{+}=-\gamma r^{4}$, with $\gamma$ a constant. We set $\gamma=f_{5} / 32$ by shifting $A$ by a constant appropriately and obtain

$$
\mathrm{e}^{4 A}=r^{4} \mathrm{e}^{4 \hat{\Delta}}, \quad \operatorname{Im} \alpha_{+}=-\frac{f_{5}}{32} r^{3} \mathrm{e}^{-\hat{\Delta}} .
$$

The first equation establishes $a$ ), and the second will be used in the proof of $d$ ). 


\section{Proof of $b)$.}

We first need to recall some facts about the grading defined by a generalized almost complex structure on differential forms (see [9] for more details). A generalized structure $\mathcal{J}$ is equivalent to the canonical pure spinor line bundle $U^{3}$ generated by $\Omega$. Acting with elements of the annihilator space $\bar{L}_{\Omega}$ of $\bar{\Omega}$ then defines the bundles $U^{3-k}=\wedge^{k} \bar{L}_{\Omega} \otimes U^{3}$, which have eigenvalues $\mathrm{i} k$ under the Lie algebra action of $\mathcal{J}$, that is $\mathcal{J} \cdot U^{k}=\mathrm{i} k U^{k}$. A generalized vector acting on an element of $U^{k}$ gives an element of $U^{k+1} \oplus U^{k-1}$. When $\mathcal{J}$ is integrable, the exterior derivative splits as $\mathrm{d}=\bar{\partial}+\partial$, with $\bar{\partial}: C^{\infty}\left(U^{k}\right) \rightarrow C^{\infty}\left(U^{k+1}\right)$ and $\partial: C^{\infty}\left(U^{k}\right) \rightarrow C^{\infty}\left(U^{k-1}\right)$.

Now the compatibility condition $\left[\mathcal{J}_{-}, \mathcal{J}_{+}\right]=0$ can be rephrased as $\mathcal{J}_{-} \cdot \Omega_{+}=0$, or $\Omega_{+} \in U^{0}$. This implies that $\mathrm{d}\left(\mathrm{e}^{-3 A} \operatorname{Im} \Omega_{+}\right)=(\bar{\partial}+\partial)\left(\mathrm{e}^{-3 A} \operatorname{Im} \Omega_{+}\right) \in U^{1} \oplus U^{-1}$, and so we can write $\mathrm{e}^{-B} F=F^{1}+F^{-1} \in U^{1} \oplus U^{-1}$. Writing $r \partial_{r}=r \partial_{r}^{+}+r \partial_{r}^{-}$with $r \partial_{r}^{ \pm}: U^{k} \rightarrow U^{k \pm 1}$, we get from $\left.r \partial_{r}\right\lrcorner\left(\mathrm{e}^{-B} F\right)=0$ that $\left.\left.r \partial_{r}^{+}\right\lrcorner F^{1}=r \partial_{r}^{-}\right\lrcorner F^{-1}=0$. The Lie algebra action of $\xi$ on generalized spinors is given by $\left.\xi \cdot=\mathcal{J}_{-}\left(r \partial_{r}\right) \cdot=\left[\mathcal{J}_{-} \cdot, r \partial_{r}\right\lrcorner\right]$. We can then calculate

$$
\begin{aligned}
\xi \cdot \mathcal{J}_{-} \cdot\left(\mathrm{e}^{-B} F\right) & \left.=\mathcal{J}_{-} \cdot r \partial_{r}\right\lrcorner \mathcal{J}_{-} \cdot\left(\mathrm{e}^{-B} F\right) \\
& \left.=\mathrm{i} \mathcal{J}_{-} \cdot\left[\left(r \partial_{r}^{+}+r \partial_{r}^{-}\right)\right\lrcorner\left(F^{1}-F^{-1}\right)\right] \\
& \left.\left.=\mathrm{i} \mathcal{J}_{-} \cdot\left(r \partial_{r}^{-}\right\lrcorner F^{1}-r \partial_{r}^{+}\right\lrcorner F^{-1}\right)=0
\end{aligned}
$$

where in the last step we used that $\left.\left.r \partial_{r}^{-}\right\lrcorner F^{1}-r \partial_{r}^{+}\right\lrcorner F^{-1} \in U^{0}$. Next, using the definition (2.48) we get

$$
\begin{aligned}
\mathcal{J}_{-} \cdot\left(\mathrm{e}^{-B} F\right) & =8 \mathcal{J}_{-} \cdot \mathcal{J}_{-} \cdot \mathrm{d}\left(\mathrm{e}^{-3 A} \operatorname{Im} \Omega_{+}\right) \\
& =-8 \mathrm{~d}\left(\mathrm{e}^{-3 A} \operatorname{Im} \Omega_{+}\right) \\
& =32 \mathrm{e}^{-3 A} \mathrm{~d} A \wedge \operatorname{Im} \Omega_{+}-8 \mathrm{e}^{-4 A} \mathrm{~d}\left(\mathrm{e}^{A} \operatorname{Im} \Omega_{+}\right) \\
& =32 \mathrm{e}^{-3 A} \mathrm{~d} A \wedge \operatorname{Im} \Omega_{+}-\mathrm{e}^{-B} \star \lambda(F)
\end{aligned}
$$

Notice that we can write down two independent annihilators of $\Omega_{+}$:

$$
\begin{aligned}
\left(1-\mathrm{i} \mathcal{J}_{+}\right) r \partial_{r} & =r \partial_{r}-\mathrm{ie}^{2 \hat{\Delta}} \eta \\
\left(1-\mathrm{i} \mathcal{J}_{+}\right) \mathrm{e}^{2 \hat{\Delta}} \mathrm{d} \log r & =\mathrm{e}^{2 \hat{\Delta}} \mathrm{d} \log r-\mathrm{i} \xi
\end{aligned}
$$

and from the fact that these are null isotropic generalized vectors (see Appendix B of [9]), we obtain the useful relations

$$
\begin{gathered}
\left.\left.\xi_{\mathrm{v}}\right\lrcorner \mathrm{d} \log r=\eta_{\mathrm{v}}\right\lrcorner \mathrm{d} \log r= \\
\left.\xi_{\mathrm{v}}\right\lrcorner \eta_{\mathrm{f}}+\eta_{\mathrm{f}}=\eta_{\mathrm{v}} \xi_{\mathrm{f}}=1
\end{gathered}
$$


Acting on (2.52) with $\xi$ gets rid of the left-hand side because of (2.51), and acting with $\mathrm{d} \log r$ gets rid of the last term because $\left.r \partial_{r}\right\lrcorner F=0$ implies $\star \lambda(F)=\mathrm{d} \log r \wedge \cdots$. Then using the annihilator constraint $\xi \cdot \operatorname{Im} \Omega_{+}=-\mathrm{e}^{2 \hat{\Delta}} \mathrm{d} \log r \wedge \operatorname{Re} \Omega_{+}$, we are left with

$$
\left.\left(\xi_{\mathrm{v}}\right\lrcorner \mathrm{d} A\right) \mathrm{d} \log r \wedge \operatorname{Im} \Omega_{+}=0,
$$

from which we conclude, since the zero-form part of $\operatorname{Im} \Omega_{+}$is non-zero by (2.50), that $\left.\xi_{\mathrm{v}}\right\lrcorner \mathrm{d} A=0$ and so $\left.\xi_{\mathrm{v}}\right\lrcorner \mathrm{d} \hat{\Delta}=0$.

We are now in a position to show that $\Omega_{+}$is invariant under $\xi$. Using the fact that the differential conditions for the real and imaginary parts of $\Omega_{+}$combine into the complex condition

$$
\mathrm{d} \Omega_{+}=\mathrm{d} A \wedge \bar{\Omega}_{+}+\frac{\mathrm{i}}{8} \mathrm{e}^{3 A} \mathrm{e}^{-B} \star \lambda(F)
$$

we obtain

$$
\begin{aligned}
\mathcal{L}_{\xi} \Omega_{+} & =\xi \cdot \mathrm{d} \Omega_{+}+\mathrm{d}\left(\xi \cdot \Omega_{+}\right) \\
& =\left(\xi \cdot+\mathrm{ie}^{2 \hat{\Delta}} \mathrm{d} \log r \wedge\right)\left[\mathrm{d} A \wedge \bar{\Omega}_{+}+\frac{\mathrm{i}}{8} \mathrm{e}^{3 A} \mathrm{e}^{-B} \star \lambda(F)\right]+\mathrm{id} \log r \wedge \mathrm{de}^{2 \hat{\Delta}} \wedge \Omega_{+} \\
& =2 \mathrm{ie}^{2 \hat{\Delta}} \mathrm{d} \log r \wedge(\mathrm{d} \hat{\Delta}-\mathrm{d} A) \wedge \Omega_{+}=0
\end{aligned}
$$

This implies $\mathcal{L}_{\xi} \mathcal{J}_{+}=0$, and since we already know that $\mathcal{L}_{\xi} \mathcal{J}_{-}=0$, we conclude that the generalized Reeb vector field $\xi$ preserves the generalized metric, $\mathcal{L}_{\xi} G=0$, or in terms of the metric $g$ and the two-form $B$,

$$
\mathcal{L}_{\xi_{\mathrm{v}}} g=\mathcal{L}_{\xi_{\mathrm{v}}} B-\mathrm{d} \xi_{\mathrm{f}}=0
$$

Finally, using (2.48) $(\underline{2.52}),\left(\mathrm{e}^{2 \hat{\Delta}} \mathrm{d} \log r-\mathrm{i} \xi\right) \cdot \Omega_{+}=0$, and $\mathrm{d}\left(\mathrm{e}^{-A} \operatorname{Re} \Omega_{+}\right)=0$, we calculate

$$
\begin{aligned}
\mathcal{L}_{\xi}\left(\mathrm{e}^{4 A} \mathrm{e}^{-B} \star \lambda(F)\right) & =\mathrm{d}\left[\xi \cdot\left(\mathrm{e}^{4 A} \mathrm{e}^{-B} \star \lambda(F)\right)\right] \\
& =4 \mathrm{~d}\left[\mathrm{de} \mathrm{e}^{4 \hat{\Delta}} \wedge \mathrm{d} r^{2} \wedge \mathrm{e}^{-A} \operatorname{Re} \Omega_{+}\right]=0 .
\end{aligned}
$$

Then since $\mathcal{L}_{\xi_{\mathrm{v}}} \mathrm{e}^{A}=\mathcal{L}_{\xi} \mathrm{e}^{-B}=0$ and $\mathcal{L}_{\xi_{\mathrm{v}}} g=0$, this leads to $\mathcal{L}_{\xi_{\mathrm{v}}} F=0$, or equivalently $\mathcal{L}_{\xi}\left(\mathrm{e}^{-B} F\right)=0$.

Proof of $c)$.

From $\mathrm{d}\left(\mathrm{e}^{-A} \operatorname{Re} \Omega_{+}\right)=0$, and the fact that $\Omega_{+}$is homogeneous degree three immediately implies $\mathcal{L}_{r \partial_{r}} \alpha_{+}=3 \alpha_{+}$, we obtain

$$
\operatorname{Re} \alpha_{+}=0, \quad \mathrm{~d}\left(\mathrm{e}^{-A} \operatorname{Im} \alpha_{+} \omega_{+}\right)=0, \quad \mathrm{~d} b_{+} \wedge \omega_{+}=0 .
$$


The second equation, combined with the fact that the Mukai pairing of $\Omega_{+}$is nowhere vanishing, $\left\langle\Omega_{+}, \bar{\Omega}_{+}\right\rangle=-(4 \mathrm{i} / 3)\left|\alpha_{+}\right|^{2} \omega_{+}^{3} \neq 0$, implies that the two-form

$$
\omega \equiv \mathrm{e}^{-2 \hat{\Delta}} r^{2} \omega_{+}
$$

is closed and non-degenerate, and hence symplectic. The justification for the presence of $\mathrm{e}^{-A}$ in the differential condition for $\operatorname{Re} \Omega_{+}$is that it leads to a symplectic form $\omega$ which is homogeneous of degree two, as usual for a symplectic cone. We may thus globally write $\omega \equiv \mathrm{d}\left(r^{2} \sigma\right) / 2$ for a real one-form $\sigma$, called the contact form, which is basic with respect to $\left.\left.r \partial_{r}, r \partial_{r}\right\lrcorner \sigma=r \partial_{r}\right\lrcorner \mathrm{d} \sigma=0$. Comparison with the annihilator constraint $\left.\left.r \partial_{r}\right\lrcorner \omega_{+}=\mathrm{e}^{2 \hat{\Delta}}\left(\eta_{\mathrm{f}}-\eta_{\mathrm{v}}\right\lrcorner b_{+}\right)$arising from $\left(r \partial_{r}-\mathrm{ie}^{2 \hat{\Delta}} \eta\right) \cdot \Omega_{+}=0$ leads to $\left.\sigma=\eta_{\mathrm{f}}-\eta_{\mathrm{v}}\right\lrcorner b_{+}$. From (2.54) and the annihilator constraint $\left.\xi_{\mathrm{v}}\right\lrcorner \omega_{+}=-\mathrm{e}^{2 \hat{\Delta}} \mathrm{d} \log r$, we obtain

$$
\left.\left.\xi_{\mathrm{v}}\right\lrcorner \sigma=1, \quad \xi_{\mathrm{v}}\right\lrcorner \mathrm{d} \sigma=0
$$

as expected for a contact form $\sigma$ and its associated unique Reeb vector field $\xi_{\mathrm{v}}$.

It remains to show that $\left.\left.\eta_{\mathrm{v}}\right\lrcorner b_{+}=\eta_{\mathrm{v}}\right\lrcorner b_{2}$, with $b_{2}$ a closed two-form. The fact that (2.53) annihilate $\Omega_{+}$gives $\left.\left.\mathrm{e}^{2 \hat{\Delta}} \eta_{\mathrm{v}}\right\lrcorner \omega_{+}=r \partial_{r}\right\lrcorner b_{+}$and $\left.\xi_{\mathrm{v}}\right\lrcorner b_{+}=\xi_{\text {f }}$, while the homogeneity of $\Omega_{+}$under $r \partial_{r}$ and $\xi$ implies the conditions $\mathcal{L}_{r \partial_{r}} b_{+}=0$ and $\mathcal{L}_{\xi_{\mathrm{v}}} b_{+}=\mathrm{d} \xi_{\mathrm{f}}$. This allows one to write the general ansatz

$$
\left.b_{+}=\mathrm{d} \log r \wedge \mathrm{e}^{2 \hat{\Delta}} \eta_{\mathrm{v}}\right\lrcorner \omega_{+}+b_{2}
$$

where $b_{2}$ is a real two-form with $\left.r \partial_{r}\right\lrcorner b_{2}=0$ and $\left.\left.r \partial_{r}\right\lrcorner \mathrm{d} b_{2}=\xi_{\mathrm{v}}\right\lrcorner \mathrm{d} b_{2}=0$. Since $\left.\eta_{\mathrm{v}}\right\lrcorner \mathrm{d} \log r=0$, this shows $\left.\left.\eta_{\mathrm{v}}\right\lrcorner b_{+}=\eta_{\mathrm{v}}\right\lrcorner b_{2}$. From the term in $\mathrm{d} \log r$ in $\mathrm{d} b_{+} \wedge \omega=0$, we get $\left.\mathrm{d}\left(\mathrm{e}^{2 \hat{\Delta}} \eta_{\mathrm{v}}\right\lrcorner \omega_{+}\right) \wedge \mathrm{d} \sigma+2 \mathrm{~d} b_{2} \wedge \sigma=0$, and contracting with $\xi_{\mathrm{v}}$ gives $\mathrm{d} b_{2}=0$.

Recall that in section 2.2 we performed a closed $B$-transform of $\Omega_{-}$by $b_{0}$ to put it into the product form (2.18) of a complex and a symplectic structure. This $B$ transform will similarly act on $\Omega_{+}$, and we consider that $b_{0}$ has been reabsorbed into the definition of $b_{+}$, and more precisely in its closed part $b_{2}$.

\section{Proof of $d)$.}

Statement $d$ ) is obtained from (2.50), (2.60), (2.61), and (2.63).

Note also that the condition $\left.r \partial_{r}\right\lrcorner b_{2}=0$ gives $\left.\left.3\left(z \partial_{z}+\bar{z} \partial_{\bar{z}}\right)\right\lrcorner b_{2}=\mathcal{H}_{\varphi}\right\lrcorner b_{2}$, while the annihilator constraint $\left.\left.\xi_{\mathrm{v}}\right\lrcorner b_{2}=\xi_{\text {f gives }} 3 \mathrm{i}\left(z \partial_{z}-\bar{z} \partial_{\bar{z}}\right)\right\lrcorner b_{2}=\mathrm{d} \varphi$, from which we conclude that $b_{2}$ can be expressed as

$$
\left.b_{2}=(\mathrm{d} \log r+\mathrm{d} h) \wedge \mathcal{H}_{\varphi}\right\lrcorner \tilde{b}_{2}+\mathrm{d} \psi \wedge \mathrm{d} \varphi+\tilde{b}_{2},
$$

where $\tilde{b}_{2}$ is the part of $b_{2}$ along the symplectic leaves defined by the $\mathcal{J}_{-}$foliation. 


\subsection{Differential system}

In this subsection we derive the full set of conditions implied by the compatibility of $\mathcal{J}_{-}$and $\mathcal{J}_{+}$, as well as by the Bianchi identity in (2.37). The Reeb vector field $\xi_{\mathrm{v}}$ defines a foliation of $Y$, and one can reduces these conditions to a set of conditions on the leaf space/transverse space to this foliation. We will see that this amounts to a simple differential system for three orthogonal symplectic forms on this transverse space. The only supersymmetry condition in [9] that we have not imposed is the equality of the norms of $\Omega_{+}$and $\Omega_{-}$. For Kähler cones, this condition is equivalent to the Einstein equation, and indeed directly leads to the Monge-Ampère equation in this case. Imposing this condition in the generalized setting thus leads to a supersymmetric $A d S_{5}$ solution, which in our terminology would be generalized Sasaki-Einstein 11

\subsubsection{Reduction}

Following section 4 of $[9$, we perform a local reduction of the six-dimensional cone to four dimensions with respect to the (generalized) Killing vector fields $r \partial_{r}$ and $\xi$. The pure spinors split as

$$
\begin{aligned}
& \Omega_{-}=r^{3} \mathrm{e}^{-3 \mathrm{i} \psi}(\mathrm{d} \log r-\mathrm{i} \eta) \cdot \mathrm{e}^{-b_{2}} \Omega_{1}, \\
& \Omega_{+}=r^{3}\left(1+\mathrm{ie}^{2 \hat{\Delta}} \mathrm{d} \log r \wedge \eta \cdot\right) \mathrm{e}^{-b_{2}} \Omega_{2},
\end{aligned}
$$

where the reduced pure spinors $\Omega_{1}$ and $\Omega_{2}$ are both of even type. We can immediately deduce that

$$
\mathrm{d} \varphi=0 .
$$

Indeed, $\Omega_{-}$has no terms in $\mathrm{d} \log r \wedge \mathrm{d} \psi$, whereas given the form of $b_{2}$ in (2.64) the right-hand side contains a term in $\mathrm{d} \log r \wedge \mathrm{d} \psi \wedge \mathrm{d} \varphi$. Recalling (2.27) and (2.30), we see that this gives

$$
r \partial_{r}=3\left(z \partial_{z}+\bar{z} \partial_{\bar{z}}\right), \quad \xi=\xi_{\mathrm{v}}=3 \mathrm{i}\left(z \partial_{z}-\bar{z} \partial_{\bar{z}}\right),
$$

which means that the foliation determined by $r \partial_{r}$ and $\xi_{\mathrm{v}}$ coincides with the complex transverse space of the local foliation defined by $\mathcal{J}_{-}$. Since by definition $r \partial_{r}$ and $\xi_{\mathrm{v}}$ are both global vector fields on $X$, it follows that $\partial_{z}$ is in fact also a global vector field on $X$; of course, initially it was defined only as a local vector field in $X_{0}$. Henceforth we

\footnotetext{
${ }^{11}$ Although here Einstein is meant to indicate that the Einstein equations of supergravity are satisfied, rather than $g_{Y}$ being an Einstein metric, which in general it is not.
} 
shall use the term foliation only with respect to the Reeb foliation defined by $\xi_{\mathrm{v}}$, which is a global foliation of $Y$. The above comments also imply that $b_{2}=\tilde{b}_{2}$ is a two-form on the four-dimensional transverse space to the Reeb foliation, or more precisely it is basic with respect to this foliation.

The pair of reduced pair spinors turns out to be

$$
\begin{aligned}
& \Omega_{1}=3 \mathrm{e}^{3 h} \mathrm{e}^{b_{2}+\mathrm{i} \omega_{0}} \\
& \Omega_{2}=-\mathrm{i} \frac{f_{5}}{32} \mathrm{e}^{-\hat{\Delta}} \mathrm{e}^{\mathrm{i} \mathrm{e}^{2 \hat{\Delta}} \omega_{T}},
\end{aligned}
$$

where we have defined the symplectic form $\omega_{T}$ on the transverse/local reduced space as

$$
\omega_{T} \equiv \frac{1}{2} \mathrm{~d} \sigma=\frac{1}{2} \mathcal{L}_{\mathcal{H}_{h}} b_{2}
$$

The corresponding generalized structures are

$$
\mathcal{J}_{1}=\left(\begin{array}{cc}
-\omega_{0}^{-1} b_{2} & \omega_{0}^{-1} \\
-\omega_{0}-b_{2} \omega_{0}^{-1} b_{2} & b_{2} \omega_{0}^{-1}
\end{array}\right), \quad \mathcal{J}_{2}=\left(\begin{array}{cc}
0 & \mathrm{e}^{-2 \hat{\Delta}} \omega_{T}^{-1} \\
-\mathrm{e}^{2 \hat{\Delta}} \omega_{T} & 0
\end{array}\right)
$$

The generalized structure $\mathcal{J}_{1}$ is integrable since we have $\mathrm{d} \Omega_{1}=3 \mathrm{~d} h \wedge \Omega_{1}$.

The compatibility of $\mathcal{J}_{-}$and $\mathcal{J}_{+}$reduces to the compatibility of $\mathcal{J}_{1}$ and $\mathcal{J}_{2}$ [33], which thus define a generalized metric $G_{T}$ on the transverse space with the following transverse metric $g_{T}$ and $B$-field $B_{T}$ :

$$
\begin{aligned}
g_{T} & =\mathrm{e}^{2 \hat{\Delta}} \omega_{T} b_{2}^{-1} \omega_{0} \\
B_{T} & =\mathrm{e}^{4 \hat{\Delta}} \omega_{T} b_{2}^{-1} \omega_{T}=-\omega_{0} b_{2}^{-1} \omega_{0}-b_{2} .
\end{aligned}
$$

The compatibility condition $\mathcal{J}_{1} \cdot \Omega_{2}=0$ is most easily analyzed by first performing a $B$-transform by $-b_{2}$ to put $\mathcal{J}_{1}$ in the standard symplectic form

$$
\mathrm{e}^{-b_{2}} \mathcal{J}_{1} \mathrm{e}^{b_{2}}=\left(\begin{array}{cc}
0 & \omega_{0}^{-1} \\
-\omega_{0} & 0
\end{array}\right)
$$

The equivalent compatibility condition $\left(\mathrm{e}^{-b_{2}} \mathcal{J}_{1} \mathrm{e}^{b_{2}}\right) \cdot \mathrm{e}^{-b_{2}} \Omega_{2}=0$ then gives

$$
\begin{gathered}
b_{2} \wedge \omega_{0}=b_{2} \wedge \omega_{T}=\omega_{0} \wedge \omega_{T}=0 \\
\mathrm{e}^{4 \hat{\Delta}} \omega_{T}^{2}=b_{2}^{2}-\omega_{0}^{2} .
\end{gathered}
$$

Note that $\omega_{0} \wedge \omega_{T}=0$ is already implied by $b_{2} \wedge \omega_{0}$ and the fact that $\omega_{T}=\mathcal{L}_{\mathcal{H}_{h}} b_{2} / 2$. 
To obtain the physical RR fluxes, we need to undo the closed $B$-transform by $b_{2}$ that we performed at the very beginning in section 2.2 to put $\Omega_{-}$into the local form of a complex/symplectic product (2.18) 12 We then obtain the following explicit formulae for the fluxes:

$$
\begin{aligned}
F_{1} & \left.\left.=-\frac{f_{5}}{4}\left(\mathcal{H}_{h}\right\lrcorner \mathcal{L}_{\mathcal{H}_{h}} \omega_{T}-\mathcal{H}_{\mathrm{e}^{-4 \hat{\Delta}}}\right\lrcorner b_{2}\right) \\
\left.\mathrm{e}^{-\left(B-b_{2}\right)} F\right|_{3} & \left.=\frac{f_{5}}{4}\left[\sigma \wedge \mathcal{L}_{\mathcal{H}_{h}} \omega_{T}+2\left(\mathcal{H}_{h}-\mathcal{H}_{\hat{\Delta}}\right)\right\lrcorner \omega_{T}^{2}\right], \\
\left.\mathrm{e}^{-\left(B-b_{2}\right)} F\right|_{5} & =-\frac{f_{5}}{2} \sigma \wedge \omega_{T}^{2} .
\end{aligned}
$$

The Bianchi identity $\mathrm{d}\left(\mathrm{e}^{-B} F\right)=0$ then gives a new condition:

$$
\mathcal{L}_{\mathcal{H}_{h}}\left(\mathcal{L}_{\mathcal{H}_{h}} \omega_{T}\right)=\mathcal{L}_{\mathcal{H}_{\mathrm{e}^{-4 \hat{\Delta}}}} b_{2}
$$

\subsubsection{Einstein condition}

By definition, the Mukai pairings $\left\langle\Omega_{-}, \bar{\Omega}_{-}\right\rangle$and $\left\langle\Omega_{+}, \bar{\Omega}_{+}\right\rangle$are nowhere-vanishing top degree forms on $X$, so they must be proportional:

$$
\left\langle\Omega_{-}, \bar{\Omega}_{-}\right\rangle=\mathrm{e}^{f}\left\langle\Omega_{+}, \bar{\Omega}_{+}\right\rangle \quad \text { or } \quad\left\|\Omega_{-}\right\|^{2}=\mathrm{e}^{f}\left\|\Omega_{+}\right\|^{2},
$$

with $f$ a real function independent of $r$; thus $\mathrm{e}^{f}$ is homogeneous of degree zero under $r \partial_{r}$. This leads to a corresponding relation between the lengths of $\omega_{T}$ and $\omega_{0}$. The calculation here is again most easily carried out in terms of the reduced pure spinors. Because of the factor of $\mathrm{e}^{2 \hat{\Delta}}$ in the decomposition of $\Omega_{+}$, the proportionality condition (2.79) becomes

$$
\left\langle\Omega_{1}, \bar{\Omega}_{1}\right\rangle=\mathrm{e}^{f} \mathrm{e}^{2 \hat{\Delta}}\left\langle\Omega_{2}, \bar{\Omega}_{2}\right\rangle
$$

which gives

$$
\left(\frac{96}{f_{5}}\right)^{2} \mathrm{e}^{6 h} \omega_{0} \wedge \omega_{0}=\mathrm{e}^{4 \hat{\Delta}+f} \omega_{T} \wedge \omega_{T}
$$

Note that combining this condition with the compatibility condition (2.74) we get

$$
b_{2} \wedge b_{2}=\left[1+\left(\frac{96}{f_{5}}\right)^{2} \mathrm{e}^{6 h-f}\right] \omega_{0} \wedge \omega_{0},
$$

\footnotetext{
${ }^{12} \mathrm{It}$ is a curious fact that without this transform we obtain in particular $\left.\mathrm{e}^{-B} F\right|_{5}=0$.
} 
which implies that $b_{2}$ is also non-degenerate, and hence a symplectic form on the transverse leaf space to the Reeb foliation.

Let us compare again with the standard Kähler setting. For a Kähler cone with metric $g=\mathrm{d} r^{2}+r^{2} g_{Y}$ and trivial canonical bundle, so that $\left(Y, g_{Y}\right)$ is a transversely Fano Sasakian manifold, the equal norm condition (2.79) becomes

$$
\frac{\mathrm{i}}{8} \Omega \wedge \bar{\Omega}=\frac{\mathrm{e}^{f}}{3 !} \omega^{3} \text {. }
$$

The Ricci-form is $\rho=\mathrm{i} \partial \bar{\partial} f$ and the Ricci scalar is then $R=-\triangle_{X} f$, where $\triangle_{X}$ denotes the Laplacian on $X$. When $f$ is constant the Kähler metric is Ricci-flat and hence Calabi-Yau, which means that $\left(Y, g_{Y}\right)$ is Sasaki-Einstein. Moreover, (2.83) immediately leads to the Monge-Ampère equation for such a metric. We thus refer to the condition that $f$ is a constant, which we can set to zero by rescaling, as the Einstein condition:

$$
f=0 \text {. }
$$

More physically, adding this condition to the definition of generalized Sasakian geometry implies that our structure satisfies all the supersymmetry conditions for an $A d S_{5}$ solution of type IIB supergravity [9], and in particular the Einstein supergravity equation. For such a solution, the physical dilaton is defined by the norms of the pure spinors through

$$
\left\|\Omega_{ \pm}\right\|^{2} \equiv \frac{1}{8} \mathrm{e}^{6 A-2 \phi}
$$

This allows us compute an expression for the volume-form on $Y$ in terms of the contact volume. Using

$$
\left\langle\Omega_{+}, \bar{\Omega}_{+}\right\rangle=-\mathrm{i} \frac{4}{3}\left|\alpha_{+}\right|^{2} \omega_{+}^{3}=-\mathrm{i}\left(\frac{f_{5}}{32}\right)^{2} \mathrm{e}^{4 \hat{\Delta}} r^{6} \mathrm{~d} \log r \wedge \sigma \wedge \mathrm{d} \sigma^{2},
$$

and $\operatorname{vol}_{X}=-\mathrm{e}^{6 \hat{\Delta}} \mathrm{d} \log r \wedge \operatorname{vol}_{Y}$, this gives

$$
\operatorname{vol}_{Y}=-\frac{f_{5}^{2}}{128} \mathrm{e}^{-8 \Delta} \sigma \wedge \mathrm{d} \sigma^{2}
$$

where $\Delta \equiv \hat{\Delta}-\phi / 4$, in agreement with equation (12) of [11].

\subsubsection{Symplectic triple}

We have now reduced our definition of a generalized Sasakian geometry to a simple differential system on the transverse space to the Reeb foliation of a contact manifold. 
More precisely, this system holds on $Y_{0}=\left.X_{0}\right|_{\{r=1\}}$, the open dense subset where $\Omega_{-}$ has type one. The compatibility condition, the Bianchi identity and the proportionality of the norms of the pure spinors boil down to a system of algebraic and differential equations for three transverse orthogonal symplectic forms $\omega_{0}, \omega_{1} \equiv \omega_{T}$, and $\omega_{2} \equiv b_{2}$ :

$$
\begin{array}{rlrl}
\mathrm{d} \omega_{i}=0 & & \forall i \in\{0,1,2\}, \\
\omega_{i} \wedge \omega_{j}=0 & \forall i \neq j,
\end{array}
$$

which induce the same orientation:

$$
\omega_{0} \wedge \omega_{0}=\alpha_{1} \omega_{1} \wedge \omega_{1}=\alpha_{2} \omega_{2} \wedge \omega_{2} \quad \text { nowhere zero }
$$

where the positive functions are

$$
\alpha_{1}=\left(\frac{f_{5}}{96}\right)^{2} \mathrm{e}^{4 \hat{\Delta}-6 h+f}, \quad \alpha_{2}=\left[1+\left(\frac{96}{f_{5}}\right)^{2} \mathrm{e}^{6 h-f}\right]^{-1} .
$$

This is called a "symplectic triple" in [17] and can be chosen as an orthogonal basis for the space $\Lambda^{+}$of positively oriented two-forms on the transverse leaf space of the Reeb foliation. This looks very similar to a hyper-Kähler structure on this transverse space, except that the symplectic forms here have different lengths. In particular, this fact means that the almost complex structures constructed by combining two symplectic forms are not integrable. Thus there is no (natural) integrable complex structure on this transverse space. This is the key difference to Sasakian geometry, where the corresponding transverse space is Kähler, and hence in particular both symplectic and complex.

The differential conditions are

$$
\omega_{1}=\frac{1}{2} \mathcal{L}_{\mathcal{H}_{h}} \omega_{2}, \quad \mathcal{L}_{\mathcal{H}_{h}}\left(\mathcal{L}_{\mathcal{H}_{h}} \omega_{1}\right)=\mathcal{L}_{\mathcal{H}^{-4 \hat{\Delta}}} \omega_{2},
$$

where $\left.\mathcal{H}_{h}=\omega_{0}^{-1}\right\lrcorner \mathrm{d} h$ for the real function $h$ and similarly for $\mathrm{e}^{-4 \hat{\Delta}}$.

Altogether, this set of conditions characterizes what we have called a generalized Sasakian structure, at least on the dense open subset $Y_{0} \subset Y$. As mentioned at the beginning, the type-change locus points that are limit points of $Y_{0}$ in $Y$ effectively lead to boundary conditions on the above structure, which degenerates at these limit points. We shall not analyse this in generality in this paper, but rather comment only in examples. Notice that, nevertheless, the contact structure and Reeb foliation are defined globally on $Y$. 
To obtain a generalized Sasaki-Einstein manifold, we must also impose the Einstein condition, which is now particularly simple to state:

$$
f=0
$$

\subsection{Example: $\beta$-transform of Kähler cones}

For illustration, we now present an explicit class of examples of generalized Sasakian manifolds. There are two key points here. First, these give a large family of such geometries that have varying Reeb vector fields and contain a generalized Sasaki-Einstein

geometry (with $f=0$ ) as a special case. Second, we will see that these are in a very precise sense generalizations of Kähler cones that are not Ricci-flat. Indeed, our strategy will be to perform a $\beta$-transform of the complex and symplectic structures of a cone that is Kähler but not in general Ricci-flat. Perhaps the most important issue that our paper raises is to understand better this space of generalized Sasakian structures, or more pressingly the associated space of Reeb vector fields in a given deformation class. We will content ourselves here with showing that there are non-trivial examples, with non-trivial spaces of Reeb vector fields. This will be sufficient to show that the generalized volume minimization we define in the next section is indeed a non-trivial minimization problem in general.

On $\mathbb{C}^{3}$ one can consider a Kähler cone metric that is a cone with respect to the weighted Euler vector field $r \partial_{r}=\sum_{i=1}^{3} \xi_{i} r_{i} \partial_{r_{i}}$, where the weights $\xi_{i} \in \mathbb{R}_{+}$are the components of the Reeb vector field, $\xi=\sum_{i} \xi_{i} \partial_{\phi_{i}}$. The holomorphic (3,0)-form is

$$
\Omega=\mathrm{d} z_{1} \wedge \mathrm{d} z_{2} \wedge \mathrm{d} z_{3}
$$

with standard complex coordinates $z_{i}=r_{i} \exp \left(\mathrm{i} \phi_{i}\right)$, while the Kähler form is as always $\omega=(\mathrm{i} / 2) \partial \bar{\partial} r^{2}$. A natural choice [13] for the Kähler potential in this case is $r^{2}=$ $\sum_{i} r_{i}^{2 / \xi_{i}}$, which gives

$$
\omega=\sum_{i} \frac{r_{i}^{2 / \xi_{i}}}{\xi_{i}^{2}} \mathrm{~d} \log r_{i} \wedge \mathrm{d} \phi_{i}=\frac{\mathrm{i}}{2} \sum_{i} \frac{r_{i}^{2 / \xi_{i}-2}}{\xi_{i}^{2}} \mathrm{~d} z_{i} \wedge \mathrm{d} \bar{z}_{i} .
$$

We then have

$$
\frac{\mathrm{i}}{8} \Omega \wedge \bar{\Omega}=\mathrm{e}^{f} \frac{\omega^{3}}{3 !}
$$

where the real function $f$ is given by

$$
\mathrm{e}^{f / 2}=\xi_{1} \xi_{2} \xi_{3} r_{1}^{1-1 / \xi_{1}} r_{2}^{1-1 / \xi_{2}} r_{3}^{1-1 / \xi_{3}}
$$


Note that the homogeneity condition $\mathcal{L}_{r \partial_{r}} \Omega=3 \Omega$ implies that $\xi_{1}+\xi_{2}+\xi_{3}=3$.

After a $\beta$-transform (2.6) by $\beta=\gamma\left(\partial_{\phi_{1}} \wedge \partial_{\phi_{2}}+\right.$ c.p. $)$ on the associated pair of pure spinors (2.13) (multiplied by $1 / 8$ to agree with conventions in [9]) we get

$$
\begin{aligned}
\mathrm{e}^{\beta} \Omega_{-} & =\frac{\gamma}{8} \mathrm{~d}\left(\bar{z}_{1} \bar{z}_{2} \bar{z}_{3}\right) \wedge \exp \left[\frac{1}{3 \gamma} \frac{\mathrm{d} \bar{z}_{1} \wedge \mathrm{d} \bar{z}_{2}}{\bar{z}_{1} \bar{z}_{2}}+\text { c.p. }\right] \\
\mathrm{e}^{\beta} \Omega_{+} & =-\mathrm{i} \frac{r^{3}}{8} \exp \left[\frac{\mathrm{i}}{r^{2}} \omega-\frac{\gamma}{r^{4}}\left(\frac{r_{1}^{2 / \xi_{1}} r_{2}^{2 / \xi_{2}}}{\xi_{1}^{2} \xi_{2}^{2}} \mathrm{~d} \log r_{1} \wedge \mathrm{d} \log r_{2}+\text { c.p. }\right)\right] .
\end{aligned}
$$

The exponent of $\mathrm{e}^{\beta} \Omega_{-}$can be put in the generalized Darboux form by shifting by a two-form proportional to $\mathrm{d}\left(\bar{z}_{1} \bar{z}_{2} \bar{z}_{3}\right)$. This gives

$$
\begin{aligned}
z & =\frac{\gamma}{8} z_{1} z_{2} z_{3}=\frac{\gamma}{8} r_{1} r_{2} r_{3} \mathrm{e}^{\mathrm{i}\left(\phi_{1}+\phi_{2}+\phi_{3}\right)} \\
\omega_{0} & =\mathrm{d} x_{1} \wedge \mathrm{d} y_{1}+\mathrm{d} x_{2} \wedge \mathrm{d} y_{2} \\
b_{0} & =-\frac{\xi_{1} \xi_{2} \xi_{3}}{3 \gamma} \mathrm{d} x_{1} \wedge \mathrm{d} x_{2}+\frac{3 \gamma}{\xi_{1} \xi_{2} \xi_{3}} \mathrm{~d} y_{1} \wedge \mathrm{d} y_{2}
\end{aligned}
$$

with the symplectic coordinates

$$
\begin{array}{ll}
x_{1}=\log \frac{r_{1}^{1 / \xi_{1}}}{r_{3}^{1 / \xi_{3}}}, & y_{1}=\frac{\xi_{1} \xi_{2} \xi_{3}}{3 \gamma}\left(\frac{\phi_{3}}{\xi_{3}}-\frac{\phi_{2}}{\xi_{2}}\right), \\
x_{2}=\log \frac{r_{2}^{1 / \xi_{2}}}{r_{3}^{1 / \xi_{3}}}, & y_{2}=\frac{\xi_{1} \xi_{2} \xi_{3}}{3 \gamma}\left(\frac{\phi_{1}}{\xi_{1}}-\frac{\phi_{3}}{\xi_{3}}\right) .
\end{array}
$$

The two-form $b_{2}$ is the difference of the part of $b_{+}$that is independent of $r$, which we call $b_{2}^{\prime}$, and $b_{0}: b_{2}=b_{2}^{\prime}-b_{0}$.

We can obtain the contact one-form, and so $\omega_{T}=\mathrm{d} \sigma / 2$, by contracting $\omega$ in (2.95) with the Euler vector field:

$$
\sigma=\sum_{i} \frac{r_{i}^{2 / \xi_{i}}}{\xi_{i} r^{2}} \mathrm{~d} \phi_{i}
$$

It is then straightforward to verify that the generalized Sasakian conditions hold for all values of the Reeb vector field $\xi$, namely the orthogonality of the three symplectic forms and the relations between their lengths. The condition $\mathcal{L}_{\mathcal{H}_{h}} b_{2}=\mathrm{d} \sigma$ is satisfied, and the Bianchi identity is trivial since $\hat{\Delta}=0$. However, the Einstein condition does not hold in general, and we rather have

$$
\left\|\mathrm{e}^{\beta} \Omega_{-}\right\|^{2}=\mathrm{e}^{f}\left\|\mathrm{e}^{\beta} \Omega_{+}\right\|^{2}
$$




\section{Volume minimization}

In this section we show that the Reeb vector field for a generalized Sasaki-Einstein manifold is determined by a (finite-dimensional) variational problem on a space of generalized Sasakian manifolds. Given that generalized Sasaki-Einstein manifolds provide solutions to type IIB supergravity, the relevant functional to minimize is an action whose Euler-Lagrange equations are the equations of motion for the type IIB bosonic fields on the five-dimensional compact space $Y$. We then rewrite this functional in terms of pure spinors and show that, when restricted to a space of generalized Sasakian s, it reduces to the contact volume (corresponding to the central charge of the dual SCFT). This result is precisely analogous to that in [8], and indeed generalizes it.

\subsection{Supergravity action}

In this section we construct an effective action for the bosonic fields on $Y$ in a type IIB $A d S_{5}$ background. That is, the Euler-Lagrange equations for this action give rise to the equations of motion satisfied by the fields. The latter include also the warp factor $\Delta$, which is effectively a scalar field on $Y$.

Type IIB supergravity describes the dynamics of a ten-dimensional metrid $13 g_{\mathrm{E}}$, dilaton $\phi, B$-field with curvature $H=\mathrm{d} B$, and the Ramond-Ramond potential $C=$ $C_{0}+C_{2}+C_{4}$ with field strength $F=F_{1}+F_{3}+F_{5}=(\mathrm{d}-H \wedge) C$. As already mentioned, the five-form has the particularity that it is self-dual: $\star_{10} F_{5}=F_{5}$. In terms of the convenient fields

$$
P_{1} \equiv \frac{\mathrm{i}}{2} \mathrm{e}^{\phi} \mathrm{d} C_{0}+\frac{1}{2} \mathrm{~d} \phi, \quad Q_{1} \equiv-\frac{1}{2} \mathrm{e}^{\phi} \mathrm{d} C_{0}, \quad G_{3} \equiv-\mathrm{e}^{\phi / 2} F_{3}-\mathrm{e}^{-\phi / 2} H,
$$

the equations of motion for the bosonic fields read [34, 35]

$$
\begin{aligned}
R_{M N}-\frac{1}{2} R g_{M N}= & P_{M} P_{N}^{*}+P_{N} P_{M}^{*}-g_{M N}\left|P_{1}\right|^{2} \\
& +\frac{1}{8}\left(G_{M P_{1} P_{2}} G_{N}^{* P_{1} P_{2}}+G_{N P_{1} P_{2}} G_{M}^{* P_{1} P_{2}}\right)-\frac{1}{4} g_{M N}\left|G_{3}\right|^{2} \\
& +\frac{1}{96} F_{M P_{1} P_{2} P_{3} P_{4}} F_{N} P_{1} P_{2} P_{3} P_{4} \\
D_{M} P^{M}= & -\frac{1}{24} G_{M N P} G^{M N P} \\
D_{P} G^{M N P}= & P_{P} G^{* M N P}-\frac{\mathrm{i}}{6} F^{M N P_{1} P_{2} P_{3}} G_{P_{1} P_{2} P_{3}}
\end{aligned}
$$

\footnotetext{
${ }^{13}$ The subscript indicates Einstein frame.
} 
where for a (complex) $p$-form $A_{p}$ we write

$$
\left|A_{p}\right|^{2} \equiv \frac{1}{p !} g^{M_{1} N_{1}} \cdots g^{M_{p} N_{p}} A_{M_{1} \cdots M_{p}} \bar{A}_{N_{1} \cdots N_{p}}
$$

The covariant derivative $D_{M}$, with respect to local Lorentz transformations and local $U(1)$ transformations with gauge field $Q_{M}$, acts on a field $A_{p}$ of charge $q$ as

$$
D_{M} A_{p}=\left(\nabla_{M}-\mathrm{i} q Q_{M}\right) A_{p}
$$

Here $P$ has charge 2 and $G$ charge 1 .

\subsubsection{A five-dimensional action}

We are interested in the field equations for the class of backgrounds that consist of the warped product of $A d S_{5}$ and a five-dimensional compact manifold $Y$ :

$$
g_{\mathrm{E}}=\mathrm{e}^{2 \Delta}\left(g_{A d S}+g_{Y}\right), \quad \text { or } \quad g_{M N}=\mathrm{e}^{2 \Delta}\left(g_{\mu \nu}+g_{m n}\right)
$$

The metric on $A d S_{5}$ is normalized so that the Ricci tensor is $R_{\mu \nu}=-4 g_{\mu \nu}$, which gives $R_{A d S}=-20$ for the Ricci scalar. In order to preserve the $S O(4,2)$ symmetry of $A d S_{5}$, all the fields are restricted to being pull-backs of fields on the internal space $Y$, with the exception of the five-form field strength, which satisfies the Freund-Rubin ansatz (2.40), given in components as

$$
F_{M N P Q R}=f_{5}\left(\varepsilon_{\mu \nu \lambda \rho \sigma}+\varepsilon_{m n p q r}\right) .
$$

In particular, $\Delta$ in (3.5) is a function on $Y$.

Let us first analyse the Einstein equation in (3.2). Recall that under a Weyl rescaling $g=\mathrm{e}^{2 \alpha} \bar{g}$ in $D$ dimensions, the Ricci tensor and the Ricci scalar transform as

$$
\begin{aligned}
R_{M N}= & \bar{R}_{M N}+(D-2)\left[-\bar{\nabla}_{M} \partial_{N} \alpha+\partial_{M} \alpha \partial_{N} \alpha\right] \\
& -\left[\bar{\nabla}^{2} \alpha+(D-2)|\mathrm{d} \alpha|^{2}\right] \bar{g}_{M N}, \\
R= & \mathrm{e}^{-2 \alpha}\left[\bar{R}-2(D-1) \bar{\nabla}^{2} \alpha-(D-2)(D-1)|\mathrm{d} \alpha|^{2}\right],
\end{aligned}
$$

where $\bar{\nabla}$ denotes the Levi-Civita connection for $\bar{g}$, and the indices are contracted with $\bar{g}$. Defining $\bar{g}=g_{A d S}+g_{Y}$ we then have $\bar{R}_{M N}=R_{\mu \nu}+R_{m n}$, and the Ricci scalar is $\bar{R}=R_{A d S}+R_{Y}$. The Freund-Rubin ansatz (3.6) gives

$$
F_{M P_{1} P_{2} P_{3} P_{4}} F_{N}^{P_{1} P_{2} P_{3} P_{4}}=4 ! f_{5}^{2}\left(-g_{\mu \nu}+g_{m n}\right)
$$


Using the above formulae, the Einstein equation then splits as

$$
\begin{aligned}
R_{\mu \nu}-\frac{1}{2} R_{Y} g_{\mu \nu}= & -\left[10+8 \nabla^{2} \Delta+28|\mathrm{~d} \Delta|^{2}+\left|P_{1}\right|^{2}+\frac{\mathrm{e}^{-4 \Delta}}{4}\left|G_{3}\right|^{2}+\frac{\mathrm{e}^{-8 \Delta} f_{5}^{2}}{4}\right] g_{\mu \nu}, \\
R_{m n}-\frac{1}{2} R_{Y} g_{m n}= & 8\left(\nabla_{m} \partial_{n} \Delta-\partial_{m} \Delta \partial_{n} \Delta\right)+P_{m} P_{n}^{*}+P_{m} P_{n}^{*} \\
& +\frac{\mathrm{e}^{-4 \Delta}}{8}\left(G_{m p_{1} p_{2}} G_{n}^{* p_{1} p_{2}}+G_{n p_{1} p_{2}} G_{m}^{* p_{1} p_{2}}\right) \\
& -\left[10+\nabla^{2} \Delta+28|\mathrm{~d} \Delta|^{2}+\left|P_{1}\right|^{2}+\frac{\mathrm{e}^{-4 \Delta}}{4}\left|G_{3}\right|^{2}-\frac{\mathrm{e}^{-8 \Delta} f_{5}^{2}}{4}\right] g_{m n},
\end{aligned}
$$

which gives the Ricci scalar on $Y$

$$
R_{Y}=\frac{100}{3}+\frac{8}{3}\left(8 \nabla^{2} \Delta+37|\mathrm{~d} \Delta|^{2}\right)+2\left|P_{1}\right|^{2}-\frac{\mathrm{e}^{-4 \Delta}}{6}\left|G_{3}\right|^{2}-\frac{5}{6} \mathrm{e}^{-8 \Delta} f_{5}^{2} .
$$

The compatibility of the two parts of the Einstein equation requires

$$
\frac{\mathrm{e}^{-4 \Delta}}{8}\left|G_{3}\right|^{2}+\frac{\mathrm{e}^{-8 \Delta} f_{5}^{2}}{4}-4=\nabla^{2} \Delta+8|\mathrm{~d} \Delta|^{2}
$$

For later reference, note that multiplying the right-hand side by $\mathrm{e}^{8 \Delta}$ and integrating by parts over $Y$ gives zero, and so we hav 14

$$
\int \mathrm{d}^{5} y \sqrt{g_{Y}} \mathrm{e}^{8 \Delta}\left(\frac{\mathrm{e}^{-4 \Delta}}{8}\left|G_{3}\right|^{2}+\frac{\mathrm{e}^{-8 \Delta} f_{5}^{2}}{4}-4\right)=0 .
$$

The equations for $P_{1}$ and $G_{3}$ can be rewritten as

$$
\begin{aligned}
\mathrm{e}^{-8 \Delta} D_{m}\left(\mathrm{e}^{8 \Delta} P^{m}\right) & =-\frac{\mathrm{e}^{-4 \Delta}}{24} G_{m n p} G^{m n p} \\
\mathrm{e}^{-8 \Delta} D_{p}\left(\mathrm{e}^{4 \Delta} G^{m n p}\right) & =\mathrm{e}^{-4 \Delta} P_{p} G^{* m n p}-\mathrm{i} \frac{\mathrm{e}^{-8 \Delta}}{6} f_{5} \varepsilon^{m n p_{1} p_{2} p_{3}} G_{p_{1} p_{2} p_{3}} .
\end{aligned}
$$

In terms of the real fields this reads

$$
\begin{aligned}
\nabla_{m}\left(\mathrm{e}^{8 \Delta+2 \phi} \partial^{m} C_{0}\right) & =-\frac{\mathrm{e}^{4 \Delta+\phi}}{6} F_{m n p} H^{m n p}, \\
\nabla_{m}\left(\mathrm{e}^{8 \Delta} \partial^{m} \phi\right) & =\mathrm{e}^{8 \Delta+2 \phi}\left|F_{1}\right|^{2}+\frac{1}{2} \mathrm{e}^{4 \Delta+\phi}\left|F_{3}\right|^{2}-\frac{1}{2} \mathrm{e}^{4 \Delta-\phi}|H|^{2}, \\
\nabla_{p}\left(\mathrm{e}^{4 \Delta+\phi} F^{m n p}\right) & =-\frac{f_{5}}{6} \varepsilon^{m n p_{1} p_{2} p_{3}} H_{p_{1} p_{2} p_{3}}, \\
\nabla_{p}\left(\mathrm{e}^{4 \Delta-\phi} H^{m n p}\right) & =\mathrm{e}^{4 \Delta+\phi} \partial_{p} C_{0} F^{m n p}+\frac{f_{5}}{6} \varepsilon^{m n p_{1} p_{2} p_{3}} F_{p_{1} p_{2} p_{3}} .
\end{aligned}
$$

\footnotetext{
${ }^{14}$ This result can also be obtained by imposing the equation of motion for $G_{3}$, or by combining the equation of motion for the warp factor $\Delta$ and the Einstein equations.
} 
All of these equations of motion can be derived from the variation of the following effective action on $Y$ :15

$$
\begin{aligned}
S_{\text {IIB }}= & \int_{Y} \mathrm{~d}^{5} y \sqrt{g_{Y}} \mathrm{e}^{8 \Delta}\left(R_{Y}-20+72|\mathrm{~d} \Delta|^{2}-\frac{1}{2}|\mathrm{~d} \phi|^{2}-\frac{1}{2} \mathrm{e}^{-4 \Delta-\phi}|H|^{2}\right. \\
& \left.-\frac{1}{2} \mathrm{e}^{2 \phi}\left|F_{1}\right|^{2}-\frac{1}{2} \mathrm{e}^{-4 \Delta+\phi}\left|F_{3}\right|^{2}+\frac{1}{2} \mathrm{e}^{-8 \Delta} f_{5}^{2}\right) \\
& +f_{5} \int_{Y} H \wedge C_{2} .
\end{aligned}
$$

This is the action with which we shall work. Notice in particular the final ChernSimons-type term.

\subsubsection{On-shell action and central charge}

We will now show that our action $S_{\text {IIB }}$ reduces on-shell, that is when supersymmetry and the equations of motion of type IIB supergravity are imposed, to the general formula in [35] for the inverse central charge of the dual SCFT. For a supersymmetric solution, this is the contact volume of $Y$, as shown in [9, 11]. In the latter context notice that going on-shell corresponds to imposing the generalized Sasakian conditions as well as the Einstein condition. This is therefore stronger than the restriction to generalized Sasakian manifolds, which is appropriate for our variational problem. We will see how to implement this in the next subsection.

When the metric is on-shell, that is when we impose the Einstein equation and hence (3.10), the action reduces to

$S_{\text {IIB }}\left(g_{Y}\right.$ on-shell $)=\int_{Y} \mathrm{~d}^{5} y \sqrt{g_{Y}} \mathrm{e}^{8 \Delta}\left(\frac{40}{3}-\frac{2}{3} \mathrm{e}^{-4 \Delta}\left|G_{3}\right|^{2}-\frac{1}{3} \mathrm{e}^{-8 \Delta} f_{5}^{2}\right)+f_{5} \int_{Y} H \wedge C_{2}$.

The Chern-Simons term can be rewritten on-shell as

$$
\begin{aligned}
f_{5} \int_{Y} H \wedge C_{2} & =\frac{f_{5}}{2 ! 3 !} \int_{Y} \mathrm{~d}^{5} y \sqrt{g_{Y}} H_{m n p} C_{q r} \varepsilon^{m n p q r} \\
& =-\frac{1}{2} \int_{Y} \mathrm{~d}^{5} y \sqrt{g_{Y}} C_{m n} \nabla_{p}\left(\mathrm{e}^{4 \Delta+\phi} F^{m n p}\right) \\
& =\frac{1}{2} \int_{Y} \mathrm{~d}^{5} y \sqrt{g_{Y}} \mathrm{e}^{4 \Delta+\phi} \nabla_{p} C_{m n} F^{m n p}
\end{aligned}
$$

where the second equality uses the equation of motion (3.16) contracted into $C_{m n}$. On

\footnotetext{
${ }^{15}$ Notice that to obtain a canonical Einstein term $\sqrt{g^{\prime}} R^{\prime}$, one has to rescale the metric as $g_{Y}=$ $\mathrm{e}^{-16 \Delta / 3} g^{\prime}$
} 
the other hand, we have

$$
\begin{aligned}
\mathrm{e}^{4 \Delta}|G|^{2}= & \mathrm{e}^{4 \Delta+\phi}\left|F_{3}\right|^{2}+\mathrm{e}^{4 \Delta-\phi}|H|^{2} \\
= & \mathrm{e}^{4 \Delta+\phi} \nabla_{m} C_{n p} F^{m n p}-C_{0} \frac{\mathrm{e}^{4 \Delta+\phi}}{3} F_{m n p} H^{m n p} \\
& +2 \mathrm{e}^{8 \Delta+2 \phi} \partial_{m} C_{0} \partial^{m} C_{0}-2 \nabla_{m}\left(\mathrm{e}^{8 \Delta} \partial^{m} \phi\right) \\
= & \mathrm{e}^{4 \Delta+\phi} \nabla_{m} C_{n p} F^{m n p}+2 \nabla_{m}\left[\mathrm{e}^{8 \Delta}\left(\mathrm{e}^{2 \phi} C_{0} \partial^{m} C_{0}-\partial^{m} \phi\right)\right],
\end{aligned}
$$

where we have used (3.15) in going from the first line to the second, and (3.14) from the second to the last. When integrated over $Y$, the total divergence vanishes so that the Chern-Simons term gives on-shell

$$
f_{5} \int_{Y} H \wedge C_{2}=\int_{Y} \mathrm{~d}^{5} y \sqrt{g_{Y}} \frac{\mathrm{e}^{4 \Delta}}{2}\left|G_{3}\right|^{2} .
$$

Using also (3.12) we obtain

$$
S_{\text {IIB }}(\text { on-shell })=8 \int_{Y} \mathrm{~d}^{5} y \sqrt{g_{Y}} \mathrm{e}^{8 \Delta}
$$

For a supersymmetric solution we now also have (2.87), and hence obtain the result that the supersymmetric on-shell $S_{\text {IIB }}$ is proportional to the contact volume of $Y$ :

$$
S_{\text {IIB }}(\text { on-shell })=-\frac{f_{5}^{2}}{16} \int_{Y} \sigma \wedge \mathrm{d} \sigma \wedge \mathrm{d} \sigma .
$$

\subsection{Restriction to generalized Sasakian manifolds}

In order to set up the variational problem, we would like to obtain an expression for $S_{\text {IIB }}$ when it is not necessarily fully on-shell, in the sense that the generalized Sasakian conditions are imposed but the Einstein condition is lifted. This is analogous to the (two rather different) computations of the Einstein-Hilbert action restricted to a space of Sasakian metrics in [8, 12], and indeed generalizes these computations to general backgrounds with all fluxes activated. Following the latter references, we first need to rewrite $S_{\text {IIB }}$ as an integral over a finite segment of the six-dimensional cone $X$, and express the integrand in terms of the pure spinors $\Omega_{ \pm}$.

Before starting the computation, we should begin by clarifying how we relate the fields in the action (3.18) to the generalized Sasakian structures we have defined in section 2. A generalized Sasakian structure involves choosing compatible pure spinors $\Omega_{ \pm}$on the cone $X$, and these in particular then define a Riemannian metric $g_{X}$ of the form (2.34) and $B$-field that is basic with respect to $r \partial_{r}$, thus leading to a metric $g_{Y}$, 
$B$-field and scalar function $\hat{\Delta}$ on $Y$. The RR fluxes $F$ are then defined in terms of the generalized structure via (2.38). Since the Bianchi identity $\mathrm{d}\left(\mathrm{e}^{-B} F\right)=0$ is part of our definition of generalized Sasakian structure, we may hence introduce RR potentials $C$. This then defines all the quantities in the action (3.18), except for the warp factor $\Delta$ and dilaton $\phi$. Instead the generalized Sasakian structure gives us a function $\hat{\Delta}$; we shall give the relation between these functions below.

We also make some mild topological assumptions, which conveniently bypass some of the subtleties involved in defining integrals of forms that are not gauge invariant 16 It is convenient to assume that $b_{1}(Y)=0$, so that $F_{1}=\mathrm{d} C_{0}$ holds for a globally defined potential $C_{0}$ on $Y$. This is a necessary condition in the Sasaki-Einstein case, by Myers' theorem, and every known supersymmetric $A d S_{5}$ solution also satisfies this condition. Without this assumption, one has to be a little more careful about global issues in the integrations by parts that will follow. In fact we have tacitly already assumed that the $B$-field is a globally defined two-form in writing the original supersymmetry conditions in the form (2.1). This is in fact a mild assumption, since in [9] it is shown that the differential form $H$ is always exact for any supersymmetric $A d S_{5}$ solution. More precisely, it was shown there that the quantity $B-b_{2}$, which is what we shall integrate by parts below, may be expressed in terms of globally-defined spinor bilinears. This leaves the possibility of adding to $B$ a discrete torsion $B$-field, which we shall again suppress. In any case, as we have defined a generalized Sasakian structure, $B$ is a globally defined two-form on $Y$, since both $\Omega_{ \pm}$were defined as global differential forms. More generally there can also be a topological twisting by a gerbe, on which $B$ is a connection - we refer to [9] for a more detailed discussion in the current context. A similar comment applies also to the RR potential $C_{2}$ - see the discussion in section 3.1 of [9]. Of course, we are then only interested in generalized Sasakian structures with these global properties also, since a continuous deformation of such a structure cannot change the topological class of these objects.

We begin by rewriting the Chern-Simons term. By a succession of integrations by parts, bearing in mind the above comments that $C_{0}, B$ and $C_{2}$ are all global forms on $Y$, we obtain

$$
f_{5} \int_{Y} H \wedge C_{2}=\left.f_{5} \int_{Y} \mathrm{e}^{-\left(B-b_{2}\right)} F\right|_{5}-F_{5}-\frac{1}{2} \mathrm{~d}\left[C_{0}\left(B-b_{2}\right)^{2}\right] .
$$

The integral on $Y$ of the exact term vanishes on using Stokes' theorem and the above

\footnotetext{
${ }^{16}$ This is really just to avoid such issues entirely; we do not believe the following assumptions are necessary.
} 
global comments. Using also the formulae $F_{5}=f_{5} \operatorname{vol}_{Y}$ and $\left.\mathrm{e}^{-\left(B-b_{2}\right)} F\right|_{5}=-\left(f_{5} / 2\right) \sigma \wedge$ $\omega_{T}^{2}=\left(16 / f_{5}\right) \mathrm{e}^{8 \Delta} \mathrm{vol}_{Y}$ from (2.77) and (2.87), we get

$$
f_{5} \int_{Y} H \wedge C_{2}=\int_{Y}\left(16 \mathrm{e}^{8 \Delta}-f_{5}^{2}\right) \operatorname{vol}_{Y}
$$

This agrees with the calculation (18) in [11] 17 Inserting this form of the Chern-Simons term into $S_{\text {IIB }}$ then gives

$$
\begin{aligned}
S_{\mathrm{IIB}}=\int_{Y} \mathrm{~d}^{5} y \sqrt{g_{Y}} \mathrm{e}^{8 \Delta} & \left(R_{Y}-4+72|\mathrm{~d} \Delta|^{2}-\frac{1}{2}|\mathrm{~d} \phi|^{2}-\frac{1}{2} \mathrm{e}^{-4 \Delta-\phi}|H|^{2}\right. \\
& \left.-\frac{1}{2}\left(\mathrm{e}^{2 \phi}\left|F_{1}\right|^{2}+\mathrm{e}^{-4 \Delta+\phi}\left|F_{3}\right|^{2}+\mathrm{e}^{-8 \Delta} f_{5}^{2}\right)\right) .
\end{aligned}
$$

We now want to write the action $S_{\text {IIB }}$, expressed in (3.18) and (3.26) in terms of the warped metric $\mathrm{e}^{2 \Delta} g_{Y}$, as an integral on the cone $X$ with metric $g_{X}$, or rather its truncation at $r=1, X_{1} \equiv[0,1] \times Y$. The metrics on $X$ and $Y$ are related through (2.34), which we repeat here for convenience

$$
g_{X}=\mathrm{e}^{2 \hat{\Delta}} r^{-2}\left(\mathrm{~d} r^{2}+r^{2} g_{Y}\right)
$$

Note that the metric $g_{\mathrm{E}}$ in (3.5) is in the Einstein frame, whereas in the application of generalized geometry to type IIB the metric $g_{X}$ is in the string frame. The two are hence related by the Weyl rescaling, $g_{X}=\mathrm{e}^{\phi / 2} g_{\mathrm{E}}$, which introduces the dilaton $\phi$. This then implies $\hat{\Delta}=\Delta+\phi / 4$, relating the generalized Sasakian function $\hat{\Delta}$ to this particular combination of the physical fields $\Delta$ and $\phi$. Using that $r^{2} \bar{R}_{X}=R_{Y}-20$ for a metric $\bar{g}_{X}=\mathrm{d} r^{2}+r^{2} g_{Y}$, and performing a Weyl rescaling by $\mathrm{e}^{2 \hat{\Delta}} r^{-2}$, we get

$$
R_{Y}-20=\mathrm{e}^{2 \hat{\Delta}}\left(R_{X}+10 \nabla^{2} \hat{\Delta}-20|\mathrm{~d} \hat{\Delta}|^{2}-20 \mathrm{e}^{-2 \hat{\Delta}}\right) .
$$

The functional can now be written as an integral over $X_{1}$ :

$$
\begin{aligned}
S_{\mathrm{IIB}}=6 \int_{X_{1}} r^{6} \mathrm{~d} r \mathrm{~d}^{5} y & \sqrt{g_{X}} \mathrm{e}^{4 \Delta-\phi}\left(R_{X}-\frac{1}{2}|H|^{2}-16 \mathrm{e}^{-2 \hat{\Delta}}\right. \\
& \left.+12|\mathrm{~d} A|^{2}-16 \mathrm{~d} A \cdot \mathrm{d} \phi+4|\mathrm{~d} \phi|^{2}-\frac{1}{2} \mathrm{e}^{2 \phi}|F|^{2}\right),
\end{aligned}
$$

${ }^{17}$ Remember that in string theory the five-form flux $F_{5}+H \wedge C_{2}$ is quantized:

$$
\int_{Y} F_{5}+H \wedge C_{2}=\left(2 \pi l_{s}\right)^{4} g_{s} N .
$$

Since $F_{5}+H \wedge C_{2}=\mathrm{d} C_{4}$, the potential $C_{4}$ is an example of a necessarily non-globally defined RR potential. This is true of course even in the Einstein case. The vanishing of the integral of the exact term in (3.24) implies that $\left.\mathrm{e}^{-\left(B-b_{2}\right)} F\right|_{5}$ satisfies the same quantization condition. 
where $|F|^{2}=\left|F_{1}\right|^{2}+\left|F_{3}\right|^{2}+\left|F_{5}\right|^{2}$.

A general formula appeared in [36] for the combination $R_{X}-H^{2} / 2$ of the Ricci scalar on $X$ and the kinetic term of the $H$-flux. Here the latter are defined via the generalized metric (2.3) associated to a pair of compatible pure spinors $\Phi$ and $\Psi$ with equal norms, $\|\Phi\|^{2}=\|\Psi\|^{2}$. In our notation the expression in [36] reads 18

$$
\begin{aligned}
R_{X}-\frac{1}{2} H^{2}= & 32 \mathrm{e}^{2 \phi-6 A}\left[|\mathrm{~d} \Phi|_{B}^{2}+\mathrm{e}^{2 A}\left|\mathrm{~d}\left(\mathrm{e}^{-A} \operatorname{Re} \Psi\right)\right|_{B}^{2}+\mathrm{e}^{-2 A}\left|\mathrm{~d}\left(\mathrm{e}^{A} \operatorname{Im} \Psi\right)\right|_{B}^{2}\right. \\
& \left.\quad+32\left|\frac{\langle\Psi, \mathrm{d} \Phi\rangle}{\operatorname{vol}_{X}}\right|^{2}+32\left|\frac{\langle\bar{\Psi}, \mathrm{d} \Phi\rangle}{\mathrm{vol}_{X}}\right|^{2}\right] \\
& +28 \mathrm{~d} A^{2}+4 \mathrm{~d} \phi^{2}-20 \mathrm{~d} A \cdot \mathrm{d} \phi+10 \nabla^{2} A-4 \nabla^{2} \phi \\
& +4(\mathrm{~d} \phi-2 \mathrm{~d} A) \cdot\left(u_{\mathrm{R}}^{1}+u_{\mathrm{R}}^{2}\right)-2 \nabla^{m}\left(u_{\mathrm{R}}^{1}+u_{\mathrm{R}}^{2}\right)_{m}+4\left[\left(u_{\mathrm{R}}^{1}\right)^{2}+\left(u_{\mathrm{R}}^{2}\right)^{2}\right]
\end{aligned}
$$

where the one-forms $u_{\mathrm{R}}^{1,2} \equiv\left(u_{m}^{1,2}+u_{m}^{* 1,2}\right) \mathrm{d} x^{m}$ can be expressed as

$$
\begin{aligned}
u_{m}^{1} & =\frac{\left\langle\gamma_{m}^{B} \bar{\Phi}, \mathrm{d} \Phi\right\rangle}{2\langle\Phi, \bar{\Phi}\rangle}+\mathrm{e}^{A} \frac{\left\langle\gamma_{m}^{B} \bar{\Psi}, \mathrm{d}\left(\mathrm{e}^{-A} \operatorname{Re} \Psi\right)\right\rangle}{\langle\Psi, \bar{\Psi}\rangle} \\
u_{m}^{2} & =\frac{\left\langle\bar{\Phi} \gamma_{m}^{B}, \mathrm{~d} \Phi\right\rangle}{2\langle\Phi, \bar{\Phi}\rangle}+\mathrm{e}^{A} \frac{\left\langle\Psi \gamma_{m}^{B}, \mathrm{~d}\left(\mathrm{e}^{-A} \operatorname{Re} \Psi\right)\right\rangle}{\langle\Psi, \bar{\Psi}\rangle}
\end{aligned}
$$

Here the norms define the combination of functions $\|\Phi\|^{2}=\|\Psi\|^{2} \equiv \frac{1}{8} \mathrm{e}^{6 A-2 \phi}$, as in (2.85) which holds on-shell, and we have defined (omitting the Clifford map slashes)

$$
\begin{aligned}
& \left.\gamma_{m}^{B} \Phi_{k} \equiv \mathrm{e}^{-B}\left(\gamma_{m} \mathrm{e}^{B} \Phi_{k}\right)=\mathrm{e}^{-B}\left[\left(\mathrm{~d} x^{m} \wedge+g^{m n} \partial_{n}\right\lrcorner\right) \mathrm{e}^{B} \Phi_{k}\right] \\
& \left.\Phi_{k} \gamma_{m}^{B} \equiv \mathrm{e}^{-B}\left(\mathrm{e}^{B} \Phi_{k} \gamma_{m}\right)=(-1)^{k} \mathrm{e}^{-B}\left[\left(\mathrm{~d} x^{m} \wedge-g^{m n} \partial_{n}\right\lrcorner\right) \mathrm{e}^{B} \Phi_{k}\right]
\end{aligned}
$$

Now recall that without imposing the Einstein condition (2.84) our pure spinors $\Omega_{ \pm}$ do not have equal norms, but satisfy instead $\left\|\Omega_{-}\right\|^{2}=\mathrm{e}^{f}\left\|\Omega_{+}\right\|^{2}$. We thus choose $\Phi=\mathrm{e}^{-f / 2} \Omega_{-}$and $\Psi=\Omega_{+}$. The pure spinor $\Phi$ is not closed, but nevertheless defines an integrable generalized almost complex structure since

$$
\mathrm{d} \Phi=-\frac{1}{2} \mathrm{~d} f \wedge \Phi
$$

Note that whenever $\Phi$ is integrable, the second line in (3.30) vanishes by compatibility.

When the differential constraints (2.1) on $\Omega_{ \pm}$are taken into account many terms cancel and we are left with

$$
\begin{aligned}
R_{X}-\frac{1}{2} H^{2}= & -\frac{1}{2} \mathrm{e}^{2 \phi}|F|^{2}+28|\mathrm{~d} A|^{2}+4|\mathrm{~d} \phi|^{2}-20 \mathrm{~d} A \cdot \mathrm{d} \phi+10 \nabla^{2} A-4 \nabla^{2} \phi \\
& +(4 \mathrm{~d} A-2 \mathrm{~d} \phi) \cdot \mathrm{d} f+\nabla^{2} f
\end{aligned}
$$

\footnotetext{
18 There is a typographical error in (C.3) of [36]: the term $+22(\mathrm{~d} A)^{2}$ should read $+28(\mathrm{~d} A)^{2}$. We
} thank Luca Martucci for communications about this point. 
As a check on this result, consider the case where $Y$ is Sasakian, rather than generalized Sasakian, so that $X$ is Kähler. In this case $\Delta=\phi=H=0, F=4 \mathrm{vol}_{Y}$, and this gives the correct result that $R_{X}=20+\nabla^{2} f$, where $f$ is the Ricci potential for the corresponding Kähler cone metric 19

In the expression for $S_{\text {IIB }}$ in (3.29) the Ricci scalar is multiplied by $\mathrm{e}^{4 \hat{\Delta}-2 \phi}$ and integrated over $X_{1}$. The integration of $\nabla^{2} f$ over $r$ can be performed trivially since $f$ is independent of $r$, and then integrating by parts we see that the second line cancels. Similar cancellations also happen after integrating the Laplacians of $A$ and $\phi$ and we are left with

$$
S_{\text {IIB }}=6 \int_{X_{1}} r^{6} \mathrm{~d} r \mathrm{~d}^{5} y \sqrt{g_{X}} \mathrm{e}^{4 \Delta-\phi}\left(24 \mathrm{e}^{-2 \hat{\Delta}}-\mathrm{e}^{2 \phi}|F|^{2}\right)
$$

Using the expressions (2.75) for the fluxes and the generalized Sasakian conditions, we find

$$
\begin{aligned}
|F|^{2}= & 16 \mathrm{e}^{8 \Delta} \mathrm{e}^{-10 \hat{\Delta}} \\
& \left.\left.+\frac{f_{5}^{2} \mathrm{e}^{-4 \hat{\Delta}}}{4 \operatorname{vol}_{X}} \mathrm{~d} \log r \wedge \mathrm{d} \psi \wedge \mathrm{d}\left[\frac{\mathrm{e}^{4 \hat{\Delta}}}{4}\left(\omega_{T} \wedge \mathcal{H}_{\mathrm{e}^{-4 \hat{\Delta}}}\right\lrcorner b_{2}-\mathcal{H}_{h}\right\lrcorner\left(\omega_{T} \wedge \mathcal{L}_{\mathcal{H}_{h}} \omega_{T}\right)\right)\right] .
\end{aligned}
$$

The second term produces an exact term in $S_{\text {IIB }}$, which vanishes on using Stokes' theorem when integrated over $Y$. In fact this step, although correct, is a little cavalier: notice that the above formula is really valid only on the dense open set $Y_{0} \subset Y$ where the integrable structure is of type one. Thus strictly speaking we end up with an integral over an infinitesimal boundary around the type-change locus after applying Stokes' theorem. One can then check that the integrand is smooth as one approaches the type-change locus and thus this integral is indeed zero. To see this, we note that the three-form in square brackets in (3.35) may be rewritten as

$$
\left.\frac{\mathrm{e}^{4 \hat{\Delta}}}{4}\left(\omega_{T} \wedge \frac{4}{f_{5}} F_{1}-\left(\mathcal{H}_{h}\right\lrcorner \omega_{T}\right) \wedge \mathcal{L}_{\mathcal{H}_{h}} \omega_{T}\right) .
$$

From the form of $\Omega_{+}$given in Proposition $2 d$ ), which recall is a global polyform on $X$, we see that $\mathrm{e}^{\hat{\Delta}}$ and $\left.\mathcal{H}_{h}\right\lrcorner \omega_{T}$ are in fact everywhere smooth on $Y$. Moreover, $\omega_{T}$ lifts to a global smooth two-form on $Y$, since it is $\mathrm{d} \sigma / 2$ with $\sigma$ the contact one-form. This demonstrates that the above three-form is in fact a smooth three-form on $Y$, not just on $Y_{0}$. On the other hand, certainly the function $h$ itself diverges along the type-change locus.

\footnotetext{
${ }^{19}$ The factor of 20 arises here because $R_{X}$ is the Ricci scalar not of the Kähler cone metric, but rather of the corresponding cylindrical metric that is related to it by a conformal factor of $r^{2}$.
} 
We thus finally obtain that for generalized Sasakian manifolds, the action functional is proportional to the contact volume:

$$
S_{\mathrm{IIB}}=8 \int_{Y} \mathrm{~d}^{5} y \sqrt{g_{Y}} \mathrm{e}^{8 \Delta}=-\frac{f_{5}^{2}}{16} \int_{Y} \sigma \wedge \mathrm{d} \sigma \wedge \mathrm{d} \sigma .
$$

This allows us to define a functional $Z$ which is the action $S_{\text {IIB }}$ restricted to a space of generalized Sasakian manifolds, normalized such that it gives exactly the contact volume of $Y$ divided by the volume of the round metric on $S^{5}$ :

$$
\begin{aligned}
Z & \equiv-\left.\frac{2}{f_{5}^{2} \pi^{3}} S_{\text {IIB }}\right|_{\text {gen. Sasakian }}=-\frac{16}{f_{5}^{2} \pi^{3}} \int_{Y} \mathrm{~d}^{5} y \sqrt{g_{Y}} \mathrm{e}^{8 \Delta} \\
& =\frac{1}{8 \pi^{3}} \int_{Y} \sigma \wedge \mathrm{d} \sigma \wedge \mathrm{d} \sigma=\frac{1}{\pi^{3}} \int_{Y} \sigma \wedge \frac{\omega_{T}^{2}}{2 !} .
\end{aligned}
$$

Defining the contact volume of a $(2 n-1)$-dimensional manifold $Y^{2 n-1}$ whose transverse space carries a symplectic form $\omega_{T}$ by

$$
\operatorname{Vol}_{\sigma}\left(Y^{2 n-1}\right) \equiv \int_{Y^{2 n-1}} \sigma \wedge \frac{\omega_{T}^{n-1}}{(n-1) !}
$$

we can simply write

$$
Z=\frac{\operatorname{Vol}_{\sigma}(Y)}{\operatorname{Vol}\left(S^{5}\right)}
$$

Note that in the case of Sasakian manifolds, for which the warp factor vanishes, $\Delta=0$, the notion of contact volume coincides with the ordinary notion of Riemannian volume, so for instance $\operatorname{Vol}_{\sigma}\left(S^{5}\right)=\operatorname{Vol}\left(S^{5}\right)$.

\subsection{Volume minimization: summary}

We are now in a position to outline the procedure of volume minimization for generalized Sasakian manifolds.

In the previous section we have shown that the action (3.18) for a space of supergravity fields on $Y$, restricted to generalized Sasakian structures, is precisely the contact volume $Z$. The contact volume, in turn, depends only on the Reeb vector field $\xi$. A general proof of this statement, which supersedes the proofs in [8], may be found in appendix B. The Reeb vector field $\xi$ for which a generalized Sasakian manifold is also Einstein is then a critical point of the contact volume $Z=Z(\xi)$ over the Reeb vector fields of a space of generalized Sasakian structures. As also shown in appendix 
B, $Z$ is strictly convex, and thus such a critical point is necessarily a minimum. Provided we work within a deformation class of generalized Sasakian structures, implying that the space of Reeb vector fields we are minimizing over is path-connected, then this minimum will be a global minimum. Clearly, all these statements generalize the results of [8] to general supersymmetric $A d S_{5}$ solutions of type IIB string theory, with the only constraint being that the background has non-zero D3-brane charge.

The key technical difference to the Sasakian case, which is currently also a deficiency, is that we do not yet have a good understanding of the deformation space of generalized Sasakian structures, and thus the corresponding space of Reeb vector fields over which we are to vary $Z(\xi)$. In the final part of this paper we shall make some reasonable assumptions about this, based on physical arguments in some particular examples, and show that the geometric result above indeed then agrees with the field theory $a$-maximization computation. It should be noted, however, that even in Sasakian geometry there is currently no general understanding of the deformation space. In fact a global picture may not even be necessary, depending on what one wants to show. For example, one of the motivations for [8] was to prove that the on-shell $Z$ is an algebraic number, since this is a definite prediction of $a$-maximization in field theory. As pointed out in [11], this follows in the general case for quasi-regular Reeb vector fields, which by definition generate a $U(1)$ action on $Y$, since then the on-shell $Z$ is a rational number, again as expected from field theory. What about irregular critical Reeb vector fields? Since the Reeb vector field also generates an isometry, and the isometry group of $Y$ is necessarily compact, it follows that such a Reeb vector field lies in the Lie algebra $\mathrm{t}$ of some torus $\mathbb{T}$ of rank at least two that acts isometrically on $Y$. If we assume that there is at least a one-parameter family of deformations of generalized Sasakian structures away from such a critical point, with Reeb vector fields defining a curve in $t$, then the Duistermaat-Heckman formula for the contact volume in [8] implies that the critical Reeb vector field $\xi_{*}$ is algebraic, and hence that $Z\left(\xi_{*}\right)$ is also algebraic, as desired. To see this, one notes that there is then always a nearby generalized Sasakian structure with Reeb vector field $\xi_{0}$ that is quasi-regular, and thus one can apply the Duistermaat-Heckman formula to the total space of the associated complex line bundle over the orbifold $Y / U(1)_{0}$, where $\xi_{0}$ defines the action of $U(1)_{0}$ on $Y$. This formula is then a rational function of the Reeb vector field with rational coefficients determined by certain Chern classes and weights, and thus setting its derivative to zero will give polynomial equations for $\xi_{*}$ with rational coefficients. We refer to [8] for the details.

In fact the only case over which there is complete control is the case of toric Sasakian 
structures. In this setting the original paper of [12] provides a complete description. It is worth contrasting this situation with the corresponding case in generalized geometry. Thus, as in [11, we define a toric generalized Sasakian manifold to be a generalized Sasakian manifold for which the symplectic structure on the cone is invariant under $\mathbb{T} \cong U(1)^{3}$. We also assume that the corresponding Reeb vector field lies in the Lie algebra of this torus 20 Notice that this does not imply that the whole structure is invariant under $U(1)^{3}$ - for example, the Pilch-Warner solution is a non-trivial solution with fluxes which is toric in this sense, but for which only a $U(1)^{2}=U(1)_{R} \times U(1)$ subgroup preserves the fluxes. In any case, in this setting there is a moment map $\mu$ under which the image of the cone $X$ is a strictly convex rational polyhedral cone $\mathcal{C}^{*} \subset t^{*} \cong \mathbb{R}^{3}$. This is a set of the form

$$
\mathcal{C}^{*}=\left\{y \in \mathrm{t}^{*} \mid\left\langle y, v_{a}\right\rangle \geq 0, a=1, \ldots, d\right\} \subset \mathbb{R}^{3},
$$

where the integer vectors $v_{a} \in \mathbb{Z}^{3}, a=1, \ldots, d$, are the inward normal vectors to the $d \geq 3$ faces of the polyhedral cone $\mathcal{C}^{*}$. The Reeb vector field $\xi$ then defines a hyperplane $\{\langle y, \xi\rangle=1 / 2\}$ in $\mathbb{R}^{3}$ that cuts $\mathcal{C}^{*}$ in a compact convex two-dimensional polytope, and the contact volume is simply the Euclidean volume of this polytope, as a function of $\xi$. Thus the minimization problem we are required to do involves minimizing this volume over an appropriate space of Reeb vector fields $\xi$. As explained in [13], necessarily $\xi$ lies in the interior of the dual polyhedral cone $\mathcal{C} \subset \mathrm{t}$ since $\mu(\xi)=\frac{1}{2} r^{2}$, so $\xi \in \mathcal{C}_{\text {Int }}$ is necessary. However, in the Sasakian case, the condition that the holomorphic volume form $\Omega$ has charge 3 then further restricts $\xi$ to lie in the intersection of $\mathcal{C}_{\text {Int }}$ with a hyperplane. This then leads to a well-defined volume minimization problem, with a unique (finite!) critical point $\xi_{*}$.

In the toric generalized setting, almost everything said above remains true. Thus a toric generalized Sasaki-Einstein solution is similarly obtained by minimizing the same two-dimensional polytope volume that appears above. The difference is that the space of Reeb vector fields over which one minimizes is in general different. This is related to the fact that in the generalized setting the closed pure spinor $\Omega_{-}$on the cone is required to have charge 3 under the Reeb vector field, as part of our definition of generalized Sasakian, so $\mathcal{L}_{r \partial_{r}} \Omega_{-}=3 \Omega_{-}$, or equivalently $\mathcal{L}_{\xi} \Omega_{-}=-3 \mathrm{i} \Omega_{-}$. Since $\Omega_{-}$is in general a polyform, the minimization problem in the generalized setting is naively going to be over a small space.

We shall see some examples of precisely this in section 4, Although the generalized

\footnotetext{
${ }^{20}$ The cases where this is not true form a finite and uninteresting list 37.
} 
geometry in these examples is not under good control, fortunately the physical interpretation is, and this allows us to determine the constraints on the Reeb vector field and apply volume minimization. In fact even more simple are the $\beta$-transforms:

\section{Example: $\beta$-transform of $\mathbb{C}^{3}$}

In order to be very concrete, let us return to the class of generalized Sasakian manifolds presented in section 2.5. Recall this arises from a family of generalized Kähler cone structures on $\mathbb{C}^{3}$ with Reeb vector fields in $\left(\mathbb{R}_{+}\right)^{3}$. In fact these are toric, in the above sense, and here $\left(\mathbb{R}_{+}\right)^{3}=\mathcal{C}_{\text {Int }}$. We can then calculate the contact volume as a function of the Reeb vector field $\xi$ :

$$
Z=\frac{1}{8 \pi^{3}} \int_{Y} \sigma \wedge \mathrm{d} \sigma \wedge \mathrm{d} \sigma=\frac{1}{\xi_{1} \xi_{2} \xi_{3}} .
$$

The homogeneity condition $\mathcal{L}_{r \partial_{r}} \Omega_{-}=3 \Omega_{-}$imposes that the components of the Reeb vector field $\xi=\sum_{i} \xi_{i} \partial_{\phi_{i}}$ satisfy

$$
\xi_{1}+\xi_{2}+\xi_{3}=3
$$

Notice here that everything is independent of the parameter $\gamma$. We see immediately that $Z$ is minimized for $\xi_{1}=\xi_{2}=\xi_{3}=1$, at which point $Z=1$ so that the contact volume of the generalized Sasaki-Einstein manifold $Y$ is equal to the volume of the five-sphere, $\operatorname{Vol}_{\sigma}(Y)=\operatorname{Vol}\left(S^{5}\right)=\pi^{3}$. Given the definition (2.97) of the function $f$, this then indeed corresponds to the Einstein condition $f=0$. We have thus reproduced the

result that the beta deformation of $\mathbb{C}^{3}$ does not change the supergravity central charge [21]. Of course this is physically fairly obvious, since it is a marginal deformation, but the important point is that we have reproduced this in a non-trivial way using generalized geometry.

The above result presumably extends to general beta deformations of toric Kähler cones, which could be treated as in [19, 22]. As explained above, the minimization problem involves precisely the same volume function of precisely the same polytope.

\subsection{Relation to a-maximization}

As mentioned in the introduction, volume minimization is believed to correspond to $a$-maximization in the dual $\mathcal{N}=1$ superconformal field theory. The equivalence of the 
two procedures has been proven for the case of toric Sasakian manifolds in [15], and in a very interesting and recent paper also for non-toric Sasakian manifolds as well [16]. In this subsection we briefly review the relation, and make a more general conjecture.

In [11] it was shown that for a general solution of type IIB supergravity of the form $A d S_{5} \times Y$, with $Y$ a generalized Sasaki-Einstein manifold, the contact volume of $Y$ is related to the central charge $a$ of the dual SCFT by the simple formula

$$
\frac{\operatorname{Vol}_{\sigma}(Y)}{\operatorname{Vol}\left(S^{5}\right)}=\frac{a_{\mathcal{N}=4}}{a},
$$

where $a_{\mathcal{N}=4}=N^{2} / 4$ is the central charge for $\mathcal{N}=4$ super-Yang-Mills theory with gauge group $S U(N)$ at large $N$. Moreover, it was shown in [9] that the Reeb vector field corresponds to the R-symmetry of the dual $\mathcal{N}=1$ SCFT.

Just as the contact volume is determined by the Reeb vector field, so the central charge $a$ is completely determined by the R-symmetry through [6, 38]

$$
a=\frac{3}{32}\left(3 \operatorname{Tr} R^{3}-\operatorname{Tr} R\right)
$$

Here the trace is over the fermions in the theory. More precisely, one typically computes this quantity in a UV theory that has a Lagrangian description and is believed to flow to an interacting superconformal fixed point in the IR, and then uses 't Hooft anomaly matching. For some time a major problem was identifying the correct global symmetry in such a UV description that becomes the R-symmetry in the IR. This was solved by Intriligator and Wecht in the beautiful paper [14]. The result is that, among the set of potential R-symmetries that are free of ABJ anomalies, the correct R-symmetry is that which (locally) maximizes the central charge. That is, one maximizes the trial central charge function over all admissible R-symmetries:

$$
a_{\text {trial }}=\frac{3}{32}\left(3 \operatorname{Tr} R_{\text {trial }}^{3}-\operatorname{Tr} R_{\text {trial }}\right)
$$

Of course, this immediately resembles $Z$-minimization, where one varies the contact volume as a function of the Reeb vector field. Indeed, even the condition that the superpotential has $\mathrm{R}$-charge 2 is analogous to the condition that $\Omega_{-}$has scaling dimension 3: both are immediate consequences of the supersymmetry parameters having a canonical (non-zero) R-charge.

In general even the dimensions of the spaces of trial R-charges and trial Reeb vector fields are different. However, in [15] it was shown in the toric Sasakian case that one can effectively perform the field theory $a$-maximization in two steps, the first step resolving 
the mixing with global baryonic symmetries. The upshot of this is that one obtains trial R-charges which are then functions of the Reeb vector field; that is, the field theory trial R-charges satisfy the well-established AdS/CFT formula [39, 40, 41, 42]

$$
R(\Phi)=\frac{\pi \operatorname{Vol}_{\sigma}\left(\Sigma_{3}\right)}{3 \operatorname{Vol}_{\sigma}(Y)}
$$

Here $\Phi$ is a chiral matter field which is "dual" to a supersymmetric three-subspace $\Sigma_{3} \subset Y$, and the volumes are understood in our language as contact volumes, which are thus functions of the trial Reeb vector field. More geometrically, in the Abelian mesonic moduli space $\Phi=0$ defines a conical divisor in $X$, which is then a cone over $\Sigma_{3}$. It is a non-trivial and striking fact that the trial R-charges defined this way satisfy the field theory anomaly cancellation conditions, for any choice of trial Reeb vector field. The authors of [15] then proved that

$$
Z=\frac{a_{\mathcal{N}=4}}{a_{\text {trial }}}
$$

holds as a relation between functions, with the right hand side understood as a function also of the Reeb vector field, as described above.

It is then natural to conjecture that the relation (3.48) still holds when $Y$ is generalized Sasakian but not necessarily Einstein. Of course, in general there would also be some analogue of the baryonic mixing to resolve in the dual field theory. However, in the examples we shall study in the next section there is no such mixing as there are no baryonic symmetries, and the functions will agree on the nose 21 We also note that, although the Abelian mesonic moduli space in the field theory is only a subspace of $X$ in general, namely the type-change locus of $\Omega_{-}$, it is nevertheless still true in examples that one can match chiral matter fields $\Phi$ with supersymmetric three-subspaces $\Sigma_{3}$, and that (3.47) still holds. This was demonstrated for the explicit Pilch-Warner solution in [9, 11], and we shall see it is also true of the new examples in the next section.

\section{Massive deformation of generalized conifolds}

In this section we present new examples of superconformal field theories whose dual geometries are generalized Sasaki-Einstein. They are obtained by massive deformations of quiver gauge theories describing the worldvolume theories of a stack of D3-branes

\footnotetext{
${ }^{21}$ It is a straightforward exercise to check that this is also the case in the beta deformation example, but this is somewhat trivial.
} 
located at so-called "generalized conifold" singularities. The simplest such example is the suspended pinch point (SPP) singularity, but this generalizes to an infinite family of generalized conifolds which are cones over the $L^{m, n, m}$ Sasaki-Einstein orbifolds. The mass deformation induces an RG flow, and the field theory analysis suggests that these theories flow to interacting superconformal fixed points in the IR. The mesonic moduli spaces of the corresponding SCFTs are not ( $N$ symmetrized copies of $)$ the original Calabi-Yau singularities, but rather only a subspace. Given the identification [18, 19] between the Abelian mesonic moduli space and the type-change locus $\mathscr{T}=X \backslash X_{0}$ of $\Omega_{-}$in $X$, where the geometry reduces to being Kähler, this means that the dual supergravity solution is indeed necessarily generalized Sasaki-Einstein. Notice that these theories must have a dual $A d S_{5}$ type IIB description, since they have been obtained by deformation of a Sasaki-Einstein background of type IIB. Although we do not know the explicit supergravity solutions, we will show that with some reasonable assumptions about their geometry, we have enough information to perform the generalized $Z$-minimization described in the previous section, and hence compute geometrically the central charge of the dual SCFT and the R-charges of certain three-subspaces. We then show that these agree with the dual field theory a-maximization computations, and moreover that the quantities even agree off-shell, as in (3.48).

\subsection{Massive deformation of $\mathcal{N}=4$ super-Yang-Mills theory}

Before considering mass deformations of generalized conifolds, we start by looking at a simple well-known example in order to acquire some geometric intuition.

One way to deform $\mathcal{N}=4$ super-Yang-Mills theory is by giving a mass to one of its three chiral superfields $\Phi_{i}, i=1,2,3$, in $\mathcal{N}=1$ language, which are all in the adjoint representation of $S U(N)$. The corresponding superpotential deformation is thus 22

$$
W_{m S Y M}=\Phi_{1}\left[\Phi_{2}, \Phi_{3}\right]+\frac{m_{1}}{2} \Phi_{1}^{2} .
$$

The resulting theory flows to an infrared fixed point with $\mathcal{N}=1$ supersymmetry, as argued by Leigh and Strassler [43]. After integrating out the massive field $\Phi_{1}$ by putting it on-shell, $\Phi_{1}=-\left[\Phi_{2}, \Phi_{3}\right] / m_{1}$, we obtain a quartic superpotential:

$$
W_{m \mathrm{SYM}}=\lambda_{1}\left[\Phi_{2}, \Phi_{3}\right]^{2}
$$

with $\lambda_{1}=-1 /\left(2 m_{1}\right)$. The requirement that the superpotential has R-charge 2 gives,

\footnotetext{
${ }^{22} \mathrm{An}$ overall trace is always implicit in these formulae.
} 
denoting the R-charges of the chiral superfields $\Phi_{i}$ by $R_{i}$,

$$
R_{1}=R_{2}+R_{3}=1
$$

The ABJ anomaly for the R-symmetry then vanishes automatically. The trial central charge is

$$
a_{\text {trial }}=\frac{27 N^{2}}{32} R_{2} R_{3}
$$

A local maximum is obtained for $R_{2}=R_{3}=1 / 2$, which gives

$$
\frac{a_{\mathcal{N}=4}}{a_{m S Y M}}=\frac{32}{27}
$$

Of course, in this example $a$-maximization is somewhat redundant, since the global $S U(2)$ symmetry at the fixed point in any case requires that $R_{2}=R_{3}$.

The dual geometry is known as the Pilch-Warner solution [44, 45], and involves a non-trivial metric on $S^{5}$ (given, for example, in [9]), as well as non-trivial three-form fluxes and five-form flux. It follows that topologically $X=C\left(S^{5}\right) \cong \mathbb{R}^{6}$. Although the solution is generalized complex, rather than complex, it is nevertheless convenient to write it in terms of complex coordinates on $\mathbb{R}^{6} \cong \mathbb{C}^{3}$. This structure is essentially inherited from that of the original solution before mass deformation, which is $\mathbb{C}^{3}$ with its flat Calabi-Yau metric. The complex coordinates $z_{i}, i=1,2,3$, effectively get rescaled (as the R-symmetry changes), and in polar coordinates these have weights $\xi_{i}$ and are given by

$$
\begin{array}{rlrl}
z_{1} & =r^{\xi_{1}} \sin \vartheta \mathrm{e}^{\mathrm{i} \phi_{1}}, & \xi_{1} & =3 / 2, \\
z_{2} & =r^{\xi_{2}} \cos \vartheta \cos \frac{\alpha}{2} \mathrm{e}^{\mathrm{i} \phi_{2}}, & & \xi_{2}=3 / 4, \\
z_{3}=r^{\xi_{3}} \cos \vartheta \sin \frac{\alpha}{2} \mathrm{e}^{\mathrm{i} \phi_{3}}, & \xi_{3}=3 / 4 .
\end{array}
$$

The closed pure spinor $\Omega_{-}$is given by

$$
\Omega_{-}=\sqrt{3} \frac{f_{5}}{96} \mathrm{~d} \bar{z}_{1}^{2} \wedge \mathrm{e}^{-b_{-}+\mathrm{i} \omega_{-}}
$$


with the rather complicated expression

$$
\begin{aligned}
-b_{-}+\mathrm{i} \omega_{-}= & -2 \mathrm{i} \sqrt{\frac{2 f_{5}}{3}} \frac{1}{3 r^{3}\left(r^{3}+\left|z_{1}\right|^{2}\right)}\left[-\frac{r^{3}\left(r^{3}+\left|z_{1}\right|^{2}\right)}{\bar{z}_{1}} \mathrm{~d} \bar{z}_{2} \mathrm{~d} \bar{z}_{3}\right. \\
& -z_{1}^{2} \bar{z}_{1} \mathrm{~d} \bar{z}_{2} \mathrm{~d} \bar{z}_{3}+\frac{\bar{z}_{1}^{2}}{2}\left(2 z_{1} \mathrm{~d} z_{2} \mathrm{~d} z_{3}-z_{3} \mathrm{~d} z_{2} \mathrm{~d} z_{1}+z_{2} \mathrm{~d} z_{3} \mathrm{~d} z_{1}\right) \\
+ & +r^{3 / 2}\left(\frac{1}{2}\left(z_{1} \bar{z}_{2}^{2}-\bar{z}_{1} z_{3}^{2}\right) \mathrm{d} z_{2} \mathrm{~d} \bar{z}_{3}-\frac{1}{2}\left(z_{1} \bar{z}_{3}^{2}-\bar{z}_{1} z_{2}^{2}\right) \mathrm{d} z_{3} \mathrm{~d} \bar{z}_{2}\right. \\
& +\frac{1}{4}\left(\left|z_{2}\right|^{2}+\left|z_{3}\right|^{2}\right) \mathrm{d} z_{1}\left(\bar{z}_{3} \mathrm{~d} \bar{z}_{2}-\bar{z}_{2} \mathrm{~d} \bar{z}_{3}\right) \\
& \left.\left.-\frac{1}{2}\left(\bar{z}_{1} z_{2} z_{3}+z_{1} \bar{z}_{2} \bar{z}_{3}\right)\left(\mathrm{d} z_{2} \mathrm{~d} \bar{z}_{2}-\mathrm{d} z_{3} \mathrm{~d} \bar{z}_{3}\right)\right)\right] .
\end{aligned}
$$

Notice that $z \propto \bar{z}_{1}^{2}$ corresponds to the superpotential $W_{m S Y M}$ in (4.2), provided we identify the complex coordinate $z_{1}$ with the scalar component of the chiral superfield $\Phi_{1}=-\left[\Phi_{2}, \Phi_{3}\right] / m_{1}$. Indeed, this is generally expected from the observation that the condition $\mathrm{d} z=0$ reproduces the F-term equations of the theory on the worldvolume of a probe D3-brane [18, 19]. Thus the type-change locus $\{\mathrm{d} z=0\}$ always corresponds to the mesonic moduli space of the SCFT. Here the type-change locus $\mathscr{T}=\left\{z_{1}=0\right\}$ of the pure spinor $\Omega_{-}$is a copy of $\mathbb{C}^{2} \subset \mathbb{R}^{6}$, on which $\Omega_{-}$reduces to a three-form

$$
\left.\Omega_{-}\right|_{\mathscr{T}}=\mathrm{i} \frac{\sqrt{2} f_{5}^{3 / 2}}{72} \mathrm{~d} \bar{z}_{1} \wedge \mathrm{d} \bar{z}_{2} \wedge \mathrm{d} \bar{z}_{3} .
$$

Notice in such expressions we do not mean a pull-back to $\mathscr{T}$, but rather a restriction of the bundle of forms to $\mathscr{T}$; the pull-back of a three-form to $\mathscr{T}$ will always be zero for dimensional reasons.

After shifting the exponent by a suitable two-form proportional to $\mathrm{d} z_{1}$, to put $\Omega_{-}$ in the generalized Darboux form, we obtain

$$
\omega_{0}=\frac{1}{3} \sqrt{\frac{f_{5}}{6}}\left[\frac{1}{\bar{z}_{1}^{2}}\left(2 \bar{z}_{1} \mathrm{~d} \bar{z}_{2} \wedge \mathrm{d} \bar{z}_{3}-\bar{z}_{2} \mathrm{~d} \bar{z}_{1} \wedge \mathrm{d} \bar{z}_{3}+\bar{z}_{3} \mathrm{~d} \bar{z}_{1} \wedge \mathrm{d} \bar{z}_{2}\right)+\text { c.c. }\right],
$$

while the expression for $b_{0}$ is rather complicated and we thus omit it. The symplectic form on $X \cong \mathbb{R}^{6}$ is

$$
\omega=\frac{1}{2} \sum_{i} \mathrm{~d} r_{i}^{2} \wedge \frac{\mathrm{d} \phi_{i}}{\xi_{i}},
$$

with $r_{1}=r \sin \vartheta, r_{2}=r \cos \vartheta \cos (\alpha / 2), r_{3}=r \cos \vartheta \sin (\alpha / 2)$. We have explicitly verified that all the conditions enunciated in subsection 2.4 .3 for a generalized SasakiEinstein solution are indeed satisfied by this Pilch-Warner solution, which is thus also a further check on our equations. 
Of course, in this case we know the explicit solution and hence Reeb vector field. However, we may now show how to recover some of these results without using the full solution, which is in fact quite complicated. The key observation is that (4.10) describes the standard symplectic structure on $\mathbb{R}^{6}$, as observed in [9]. In order to perform $Z$ minimization, we let the Reeb vector field $\xi=\sum_{i} \xi_{i} \partial_{\phi_{i}} \in\left(\mathbb{R}_{+}\right)^{3}$ be arbitrary in the expression (4.10) for the symplectic form, which then leads to the contact volume

$$
Z(\xi)=\frac{1}{\xi_{1} \xi_{2} \xi_{3}}
$$

Note that this is the same contact volume function (3.42) as for the beta deformation of Kähler cones on $\mathbb{C}^{3}$, since in both cases the symplectic structure on $\mathbb{R}^{6} \cong \mathbb{C}^{3}$ is the standard one. The generalized holomorphy condition $\mathcal{L}_{\xi} \Omega_{-}=-3 \mathrm{i} \Omega_{-}$gives constraints on the Reeb vector field. In particular, the three-form condition gives $\xi_{1}+\xi_{2}+\xi_{3}=3$, which is easily deduced by looking at the homogeneity of $\left.\Omega_{-}\right|_{\mathscr{T}}$, while, in contrast to the $\beta$-transform example, the one-form condition $\mathcal{L}_{\xi} \mathrm{d} \bar{z}_{1}^{2}=-3 \mathrm{id} \bar{z}_{1}^{2}$ gives the additional condition $\xi_{1}=3 / 2$, as does the five-form condition. We thus have the constraints

$$
\xi_{1}+\xi_{2}+\xi_{3}=3, \quad \xi_{1}=\frac{3}{2} .
$$

Minimizing $Z$ under these constraints indeed gives the correct Reeb vector field $\xi=$ $(3 / 2,3 / 4,3 / 4)$. Using the relation between the Reeb vector field and the R-charges, $\xi_{i} / 3=R_{i} / 2$, we see that the conditions (4.12) and (4.3) match and that the conjecture (3.48) indeed holds:

$$
Z=\frac{a_{\mathcal{N}=4}}{a_{\text {trial }}}
$$

\subsection{Suspended pinch point}

Before turning on massive deformations, we first review the gauge theory on $N$ D3branes probing the suspended pinch point singularity.

The suspended pinch point is a non-isolated hypersurface singularity given by

$$
X_{\mathrm{SPP}}=\left\{u^{2} v=w z\right\} \subset \mathbb{C}^{4}
$$

where $u, v, w, z$ are complex coordinates on $\mathbb{C}^{4}$. All such hypersurface singularities are Calabi-Yau (or, more precisely, Gorenstein), in the sense that they admit a nowhere zero holomorphic $(3,0)$-form $\Omega$ on the locus of smooth points. This particular singularity is also toric, meaning that there is a holomorphic action of $\mathbb{T}_{\mathbb{C}}=\left(\mathbb{C}^{*}\right)^{3}$ with a 
dense open orbit. It may thus be rewritten in the language of toric geometry, reviewed very briefly in section 3.3 - we also refer the reader to [12], where the suspended point point singularity is discussed in further detail. In particular, the image of $X_{\mathrm{SPP}}$ under the moment map for any choice of toric Kähler metric on $X_{\mathrm{SPP}}$ is given by a polyhedral cone $\mathcal{C}^{*}$ in $\mathbb{R}^{3}$ of the form (3.41), where the inward-pointing normal vectors are

$$
\begin{gathered}
v_{0}=(1,1,0), \quad v_{1}=(1,2,0), \quad v_{2}=(1,1,1) \\
v_{3}=(1,0,1), \quad v_{4}=(1,0,0)
\end{gathered}
$$

Here we have used the fact that for any toric Gorenstein singularity one can conveniently set the first component of the normal vectors to 1 by an appropriate $S L(3 ; \mathbb{Z})$ transformation of the torus. They are thus of the form $v_{a}=\left(1, w_{a}\right)$, where $w_{a} \in \mathbb{Z}^{2}$.

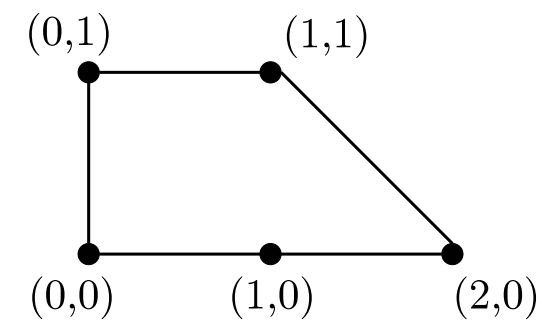

Figure 1: Toric diagram for the suspended pinch point.

Figure 1 shows the toric diagram, which is the convex hull of the $\left\{w_{a}\right\}$ in $\mathbb{R}^{2}$, or equivalently is the projection of the dual cone $\mathcal{C}$ to the plane $e_{1}=1$. The four external vertices correspond to four torus-invariant divisors $D_{\alpha}=C\left(\Sigma_{\alpha}\right), \alpha=1,2,3,4$, which are cones over three-subspaces $\Sigma_{\alpha} \subset Y_{\text {SPP. }}$ It is the additional vertex point $w_{0}=(1,0)$ on the interior of an external edge that signifies that $X_{\mathrm{SPP}}$ is not an isolated singularity - in fact there is an $A_{1}$ singularity running out of $u=v=w=z=0$, at every non-zero value of $v$. The relation between the toric and algebraic descriptions is obtained as usual by noting that the normal vectors satisfy $\sum_{\alpha=1}^{4} Q_{\alpha} v_{\alpha}=0$, with the $U(1)_{B}$ charge vector $Q=(-1,2,-2,1)$. We may then associate complex coordinates $Z_{\alpha}$ to each divisor $\Sigma_{\alpha}$, in terms of which we construct $U(1)_{B}$-invariant monomials as

$$
u=Z_{1} Z_{4}, \quad v=Z_{2} Z_{3}, \quad w=Z_{1}^{2} Z_{2}, \quad z=Z_{3} Z_{4}^{2}
$$

These generate all such invariants, and satisfy our original algebraic equation $u^{2} v=w z$. Indeed, being holomorphic functions on $X_{\mathrm{SPP}}$ of definite charge under the torus action, they define lattice points inside the cone $\mathcal{C}^{*}$, and then precisely generate its lattice 
points over $\mathbb{Z}_{\geq 0}$. Thus with this interpretation we also have

$$
u=(1,0,-1), \quad v=(0,0,1), \quad w=(0,1,0), \quad z=(2,-1,-1),
$$

being the generators of $\mathcal{C}^{*}$. We shall need these formulae later.

It was only recently that an explicit Calabi-Yau cone metric was constructed on $X_{\mathrm{SPP}}$ [23, 24]. In fact the corresponding Sasaki-Einstein orbifold metric on $Y_{\mathrm{SPP}}$ is one of these $L^{p, q, r}$ spaces, namely $L^{1,2,1}$. However, before this metric was known (and indeed known to exist), the Reeb vector field and hence volumes of $Y_{\mathrm{SPP}}$ and its supersymmetric toric subspaces $\Sigma_{\alpha}$ were computed using volume minimization in the original paper [12]. These are given by

$$
\begin{aligned}
\operatorname{Vol}(Y) & =\frac{\pi}{2 \xi_{1}} \sum_{\alpha} \operatorname{Vol}\left(\Sigma_{\alpha}\right), \\
\operatorname{Vol}\left(\Sigma_{\alpha}\right) & =2 \pi^{2} \frac{\left(v_{\alpha-1}, v_{\alpha}, v_{\alpha+1}\right)}{\left(\xi, v_{\alpha-1}, v_{\alpha}\right)\left(\xi, v_{\alpha}, v_{\alpha+1}\right)},
\end{aligned}
$$

where $(u, v, w)$ denotes the determinant of the $3 \times 3$ matrix whose rows are $u$, $v$, and $w$. With the choice of normal vectors in Figure 1 we obtain

$$
\begin{aligned}
\operatorname{Vol}\left(\Sigma_{1}\right) & =\frac{2 \pi^{2}}{\xi_{3}\left(2 \xi_{1}-\xi_{2}-\xi_{3}\right)}, \quad \operatorname{Vol}\left(\Sigma_{4}\right)=\frac{2 \pi^{2}}{\xi_{2} \xi_{3}}, \\
\operatorname{Vol}\left(\Sigma_{2}\right) & =\frac{2 \pi^{2}}{\left(\xi_{1}-\xi_{3}\right)\left(2 \xi_{1}-\xi_{2}-\xi_{3}\right)}, \quad \operatorname{Vol}\left(\Sigma_{3}\right)=\frac{2 \pi^{2}}{\xi_{2}\left(\xi_{1}-\xi_{3}\right)}, \\
Z & =\frac{2 \xi_{1}-\xi_{3}}{8 \xi_{2} \xi_{3}\left(\xi_{1}-\xi_{3}\right)\left(2 \xi_{1}-\xi_{2}-\xi_{3}\right)} .
\end{aligned}
$$

In the basis in which the normal vectors to $\mathcal{C}^{*}$ all have their first component equal to 1 , the holomorphic three-form $\Omega$ satisfies $\mathcal{L}_{\partial / \partial \phi_{1}} \Omega=i \Omega$ and $\mathcal{L}_{\partial / \partial \phi_{2,3}} \Omega=0$, so the homogeneity condition requires the first component of the Reeb vector field $\xi$ to be equal to $3[12]$ :

$$
\xi_{1}=3 .
$$

Then it is straightforward to check that $Z$ has a (global) minimum for

$$
\xi=\left(3, \frac{3+\sqrt{3}}{2}, 3-\sqrt{3}\right) .
$$

Notice that in the Sasaki-Einstein case $f_{5}=4$ and $\Delta=0$, and the contact volume $Z$ reduces to the Riemannian volume of $Y$, relative to that of the round metric on $S^{5}$, and so we have

$$
\operatorname{Vol}\left(Y_{\mathrm{SPP}}\right)=\frac{2 \pi^{3}}{3 \sqrt{3}}, \quad \operatorname{Vol}\left(\Sigma_{1,4}\right)=\frac{2 \pi^{2}}{3}, \quad \operatorname{Vol}\left(\Sigma_{2,3}\right)=\frac{4 \pi^{2}}{3+3 \sqrt{3}} .
$$




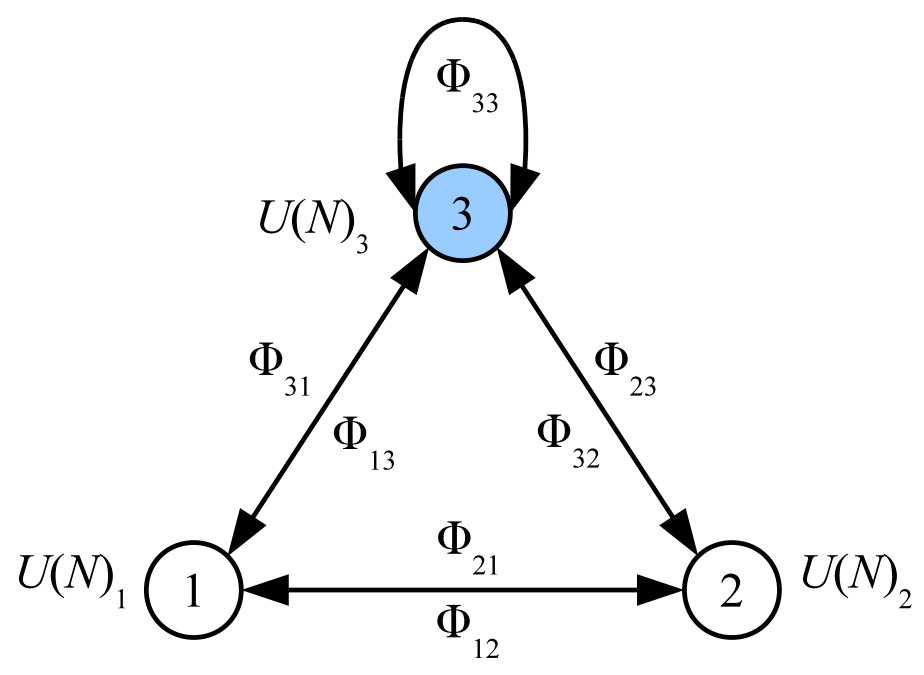

Figure 2: Quiver diagram for the gauge theory on $N$ D3-branes probing the suspended pinch point. There are three $U(N)_{i}$ gauge groups, with six bifundamental fields $\Phi_{i j}$ and one adjoint field $\Phi_{33}$.

The gauge theory on $N$ D3-branes at such a singularity was first studied by Morrison and Plesser [5] and Uranga [46]. This is of quiver form, with the quiver diagram shown in Figure 2, Here the three nodes represent three $U(N)$ gauge groups, and the arrows represent bifundamental chiral superfields. More precisely, a field $\Phi_{i j}$ connecting the $i^{\text {th }}$ node to the $j^{\text {th }}$ node is in the fundamental representation of $U(N)_{i}$ and the antifundamental of $U(N)_{j}$; the field $\Phi_{33}$ is in the adjoint representation of $U(N)_{3}$. The superpotential is

$$
W_{\mathrm{SPP}}=\Phi_{12} \Phi_{21} \Phi_{13} \Phi_{31}-\Phi_{23} \Phi_{32} \Phi_{21} \Phi_{12}+\Phi_{33}\left(\Phi_{32} \Phi_{23}-\Phi_{31} \Phi_{13}\right)
$$

Focusing on the Abelian theory with $N=1$, the resulting F-term and D-term conditions are

$$
\begin{array}{ll}
\Phi_{23} \Phi_{32}=\Phi_{13} \Phi_{31}, \quad \Phi_{33}=\Phi_{12} \Phi_{21}, & \\
\left|\Phi_{21}\right|^{2}-\left|\Phi_{12}\right|^{2}+\left|\Phi_{31}\right|^{2}-\left|\Phi_{13}\right|^{2}=0, & U(1)_{1} \\
\left|\Phi_{21}\right|^{2}-\left|\Phi_{12}\right|^{2}+\left|\Phi_{32}\right|^{2}-\left|\Phi_{23}\right|^{2}=0, & U(1)_{2} .
\end{array}
$$

Notice here that we have precisely neglected the branch of solutions to the F-term equations in which $\Phi_{23}=\Phi_{32}=\Phi_{13}=\Phi_{31}=0$, for which then $\Phi_{33}, \Phi_{12}$ and $\Phi_{21}$ are left unconstrained by the F-terms. Imposing also the D-terms on this branch leads to a copy of $\mathbb{C}^{2}$, which exists precisely because the singularity is not isolated. Ignoring 
this, which corresponds to motion of fractional branes along the residual singularity, we can construct the following $U(1)_{1,2}$-invariant monomials in the fields, which then generate the top-dimensional irreducible component of the mesonic moduli space:

$$
\begin{aligned}
u=\Phi_{23} \Phi_{32}=\Phi_{13} \Phi_{31}, & v=\Phi_{33}=\Phi_{12} \Phi_{21}, \\
w=\Phi_{13} \Phi_{32} \Phi_{21}, & z=\Phi_{12} \Phi_{23} \Phi_{31} .
\end{aligned}
$$

We see that these indeed satisfy the suspended pinch point hypersurface relation $u^{2} v=$ $w z$.

By comparing the expressions for $u, v, w, z$ in terms of the coordinates $Z_{\alpha}$ associated with the three-subspaces $\Sigma_{\alpha}$ (4.16) , and in terms of the gauge theory fields $\Phi_{i j}$ (4.27), we deduce that the vanishing locus of a field $\Phi_{i j}$ is associated with the divisors $D_{\alpha}=C\left(\Sigma_{\alpha}\right)$ as in Table 1 .

\begin{tabular}{|c|c|c|c|}
\hline 3-subspace & Fields & $Q_{B}$ & R-charge \\
\hline \hline$\Sigma_{1}$ & $\Phi_{32}, \Phi_{13}$ & -1 & $1 / \sqrt{3}$ \\
\hline$\Sigma_{2}$ & $\Phi_{21}$ & 2 & $1-1 / \sqrt{3}$ \\
\hline$\Sigma_{3}$ & $\Phi_{12}$ & -2 & $1-1 / \sqrt{3}$ \\
\hline$\Sigma_{4}$ & $\Phi_{31}, \Phi_{23}$ & 1 & $1 / \sqrt{3}$ \\
\hline$\Sigma_{2} \cup \Sigma_{3}$ & $\Phi_{33}$ & 0 & $2-2 / \sqrt{3}$ \\
\hline
\end{tabular}

Table 1: Divisors, fields, and charges for the SPP theory.

We now perform a-maximization for the superconformal fixed point of this theory, at large $N$. The requirement that the superpotential has R-charge two gives

$$
\begin{gathered}
R_{12}+R_{21}+R_{23}+R_{32}=2 \\
R_{23}+R_{32}=R_{13}+R_{31}, \quad R_{12}+R_{21}=R_{33} .
\end{gathered}
$$

Using this, one sees that anomaly cancellation is then automatically satisfied. The trial central charge is then

$$
a_{\text {trial }}=\frac{9 N^{2}}{32}\left[3+\sum_{i, j}\left(R_{i j}-1\right)^{3}\right],
$$

which is locally maximized for

$$
R_{23,32,13,31}=\frac{1}{\sqrt{3}}, \quad R_{12,21}=1-\frac{1}{\sqrt{3}}, \quad R_{33}=2-\frac{2}{\sqrt{3}} .
$$


This gives

$$
\frac{a_{\mathcal{N}=4}}{a_{\mathrm{SPP}}}=\frac{2}{3 \sqrt{3}}
$$

We now wish to compare this with $Z$-minimization already performed. The Rcharge of a dibaryonic operator $\mathcal{B}_{\alpha}=\operatorname{det} \Phi_{i j}$ arising from wrapping a D3-brane over $\Sigma_{\alpha}$ is computed using the AdS/CFT formula (3.47). Using the toric volumes above, we can see that the conditions on the R-charges are equivalent to the condition $\xi_{1}=3$, and that the contact volume $Z$ is equal to the inverse of the central charge, where one takes the trial R-charges to be functions of the trial Reeb vector $\xi$ using the volume formula (3.47):

$$
Z=\frac{a_{\mathcal{N}=4}}{a_{\text {trial }}}
$$

Of course, this was proven in generality by Butti and Zaffaroni [15].

\section{Mass deformation}

Having fairly thoroughly summarized the suspended pinch point theory, we now turn to its massive deformation. We thus consider deforming the theory by adding a mass term for the adjoint field:

$$
W_{m \mathrm{SPP}}=W_{\mathrm{SPP}}+\frac{m}{2} \Phi_{33}^{2}
$$

Integrating out the massive field by imposing its equation of motion, $\Phi_{33}=\left(\Phi_{31} \Phi_{13}-\right.$ $\left.\Phi_{32} \Phi_{23}\right) / m$, we are left with a quartic superpotential

$$
W_{m \mathrm{SPP}}=\Phi_{12} \Phi_{21} \Phi_{13} \Phi_{31}-\Phi_{23} \Phi_{32} \Phi_{21} \Phi_{12}-\lambda_{33}\left(\Phi_{32} \Phi_{23}-\Phi_{31} \Phi_{13}\right)^{2},
$$

with $\lambda=1 /(2 m)$. Neglecting the corresponding branch of the moduli space that we neglected previously (which the reader may check is a copy of $\mathbb{C}$ ), the F-terms give

$$
\Phi_{13} \Phi_{31}=\Phi_{23} \Phi_{32}, \quad \Phi_{12} \Phi_{21}=0
$$

The D-terms are the same as for the SPP theory, and we may similarly construct the gauge-invariant monomials

$$
p=\Phi_{23} \Phi_{32}=\Phi_{13} \Phi_{31}, \quad q=\Phi_{12} \Phi_{21}, \quad s=\Phi_{13} \Phi_{32} \Phi_{21}, \quad t=\Phi_{12} \Phi_{23} \Phi_{31}
$$

The F-term condition $q=0$ also enforces that either $s$ or $t$ vanishes. The moduli space is thus $\{u, s, t=0\} \cup\{u, t, s=0\} \simeq \mathbb{C}^{2} \cup_{\mathbb{C}} \mathbb{C}^{2}$; that is, two copies of $\mathbb{C}^{2}$ intersecting 
over $\mathbb{C}$. The $a$-maximization computation below suggests the existence of a non-trivial interacting IR fixed point for this theory, and then the fact that this mesonic moduli space is not a three-fold implies that the dual type IIB description must be generalized geometric, rather than a Sasaki-Einstein solution.

The R-charges at the putative IR fixed point can be determined by $a$-maximization. The condition that the superpotential has R-charge 2 gives

$$
R_{12}+R_{21}=1, \quad R_{23}+R_{32}=1, \quad R_{13}+R_{31}=1
$$

The condition of vanishing ABJ anomaly is then automatically satisfied. The trial central charge is

$$
a_{\text {trial }}=\frac{27 N^{2}}{32}\left(R_{12} R_{21}+R_{13} R_{31}+R_{23} R_{32}\right)
$$

A local maximum is obtained when all the $\mathrm{R}$-charges are equal to $1 / 2$, which gives

$$
\frac{a_{\mathcal{N}=4}}{a_{m \mathrm{SPP}}}=\frac{32}{81} \text {. }
$$

Numerically, this is slightly less than the central charge for the SPP theory,

$$
\frac{a_{m \mathrm{SPP}}}{N^{2}}=\frac{81}{128} \approx 0.63<\frac{a_{\mathrm{SPP}}}{N^{2}}=\frac{3 \sqrt{3}}{8} \approx 0.65
$$

This is then consistent with the $a$-theorem, $a_{\mathrm{IR}}<a_{\mathrm{UV}}$, which in turn is based on the intuition that we are integrating out degrees of freedom when flowing to the IR.

One of the new results in this paper is that we now have some understanding of the dual $Z$-minimization to perform on the gravity side. However, to apply this we need to make two assumptions, which are motivated by our previous examples. Firstly, we assume that the symplectic structure of $X$ is left unchanged by the massive deformation. This ensures that the toric diagram remains the same as for the original SPP singularity. This is true of the explicit Pilch-Warner solution, which is the IR fixed point of a similar massive deformation of $\mathcal{N}=4$ super-Yang-Mills. It would certainly be nice to understand better the physical significance of this. The second condition is easier to justify. Here we assume that the homogeneity condition on the pure spinor $\Omega_{-}$for the putative IIB dual requires

$$
\xi_{3}=3 / 2
$$

The reason for this is that the one-form part of the pure spinor $\Omega_{-}$is precisely related to the scalar part of the superpotential. Hence $\Omega_{-} \propto \mathrm{d} \bar{v}^{2}$, where recall that in the Abelian 
moduli space of the original SPP theory $v=\Phi_{33}$, where we deform by the mass term $m \Phi_{33}^{2} / 2$. This is indeed precisely what happens for the Pilch-Warner solution, as we reviewed in section 4.1. In the basis we have chosen one immediately sees from (4.17) that the one-form part of the homogeneity condition $\mathcal{L}_{\xi} \mathrm{d} v^{2}=3 \mathrm{id} v^{2}$ gives precisely $\xi_{3}=3 / 2$.

With the homogeneity condition $\xi_{1}=3$, the function $Z$ then reads

$$
Z=\frac{1}{2 \xi_{2}\left(9-2 \xi_{2}\right)}
$$

which is minimized at $\xi_{2}=9 / 4$. Using again (3.47), we verify the equivalence of the $Z$ and $a$ functions:

$$
Z=\frac{a_{\mathcal{N}=4}}{a_{\text {trial }}}
$$

The contact volumes of $Y_{m \mathrm{SPP}}$ and the subspaces $\Sigma_{\alpha}$ after mass deformation are

$$
\operatorname{Vol}_{\sigma}\left(Y_{m \mathrm{SPP}}\right)=\frac{32 \pi^{3}}{81}, \quad \operatorname{Vol}_{\sigma}\left(\Sigma_{\alpha}\right)=\frac{16 \pi^{2}}{27}, \quad \forall \alpha=1,2,3,4
$$

We also see that the AdS/CFT formula (3.47), which was shown to hold also for generalized geometries in [11] provided the volumes are interpreted as contact volumes, gives the correct result that the R-charge of each bifundamental field is $1 / 2$. That is,

$$
R\left(\Phi_{i j}\right)=\frac{\pi \operatorname{Vol}_{\sigma}\left(\Sigma_{\alpha}\right)}{3 \operatorname{Vol}_{\sigma}\left(Y_{m S P P}\right)}=\frac{1}{2}
$$

which acts as a further check on this result.

We have thus predicted the existence of a supersymmetric $A d S_{5}$ solution of type IIB supergravity, with the same topology and toric symplectic structure as $X_{\mathrm{SPP}}$, a Reeb vector field which in the above basis is $(3,9 / 4,3 / 2)$, a pure spinor $\Omega_{-}$with oneform component proportional to $\mathrm{d} \bar{v}^{2}$, where $v$ is the complex-valued function on $X_{\mathrm{SPP}}$ specified above, and with a corresponding type-change locus $\mathscr{T}=\mathbb{C}^{2} \cup_{\mathbb{C}} \mathbb{C}^{2}$. This is a substantial amount of information about this solution. In fact, this is essentially as much as one knows about toric Calabi-Yau solutions for which we only know that there exists a Sasaki-Einstein metric via the existence result of [47]. Our results then show that the central charges and R-charges of chiral fields for such a gravity solution and the dual field theory match using AdS/CFT. 


\subsection{Generalized conifolds}

Having studied the SPP theory and its massive deformation in detail, we turn now to a simple infinite family of generalizations of this example. Since the details are similar, we shall be more brief.

We begin with the generalized conifolds described by the hypersurface equation [46]

$$
X_{m, n}=\left\{u^{n} v^{m}=w z\right\} \subset \mathbb{C}^{4}
$$

These are again also toric, and provided $\operatorname{gcd}(m, n)=1$ the corresponding polyhedral cone $\mathcal{C}^{*}$ has primitive normal vectors

$$
v_{1}=(1, n, 0), \quad v_{2}=(1, m, 1), \quad v_{3}=(1,0,1), \quad v_{4}=(1,0,0) .
$$

The toric diagram is shown in Figure 3 .

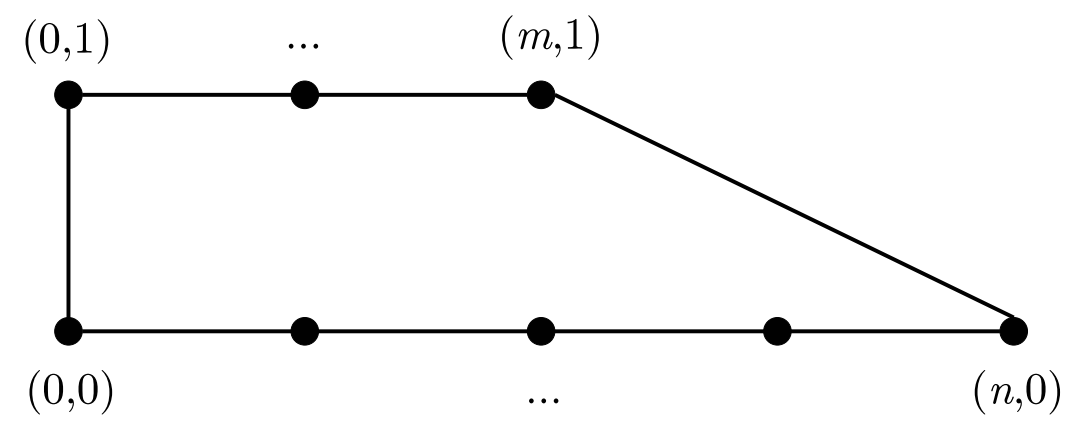

Figure 3: Toric diagram for the generalized conifold $X_{m, n}=C\left(L^{m, n, m}\right)$.

The dual cone $\mathcal{C}$ is also generated by four primitive vectors, namely

$$
u=(1,0,-1), \quad v=(0,0,1), \quad w=(0,1,0), \quad z=(n,-1, m-n)
$$

which correspond to four holomorphic functions on $X_{m, n}$ with definite charge under the torus. It is again an elementary exercise to check that these generate over $\mathbb{Z}_{\geq 0}$ all lattice points in $\mathcal{C}^{*}$. Notice that we have $\sum_{\alpha=1}^{4} Q_{\alpha} v_{\alpha}=0$, with the $U(1)_{B}$ charge vector $Q=(-m, n,-n, m)$. Writing $Z_{\alpha}, \alpha=1,2,3,4$, as coordinates on $\mathbb{C}^{4}$, then the $U(1)_{B}$ invariants are spanned by the four functions

$$
u=Z_{1} Z_{4}, \quad v=Z_{2} Z_{3}, \quad w=Z_{1}^{n} Z_{2}^{m}, \quad z=Z_{3}^{m} Z_{4}^{n},
$$

which then satisfy $u^{n} v^{m}=w z$. Again, this certainly requires $\operatorname{gcd}(m, n)=1$. 


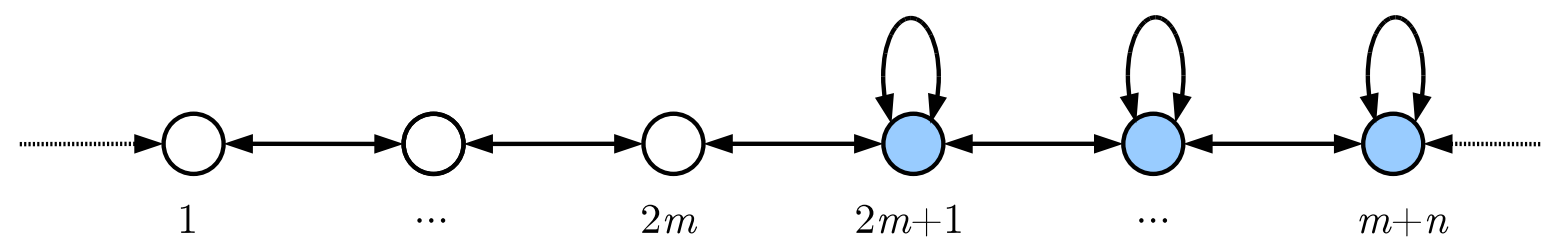

Figure 4: Quiver diagram for the gauge theory on $N$ D3-branes probing $X_{m, n}=$ $C\left(L^{m, n, m}\right)$. The dashed arrows at both extremities are identified.

These generalized conifolds are cones over the Sasaki-Einstein orbifolds $L^{m, n, m}$. The SCFT dual to $N$ D3-branes probing $X_{m, n}=C\left(L^{m, n, m}\right)$ was studied in [48]. Again, this is the IR limit of a quiver gauge theory, now with $N_{g}=m+n U(N)$ gauge group factors, the last $n-m$ of which have an adjoint field. The quiver is shown in Figure 4 , and the superpotential is

$$
W_{L^{m, n, m}}=\sum_{i=1}^{2 m}(-)^{i} \Phi_{i, i-1} \Phi_{i-1, i} \Phi_{i, i+1} \Phi_{i+1, i}+\sum_{i=2 m+1}^{n-m} \Phi_{i, i}\left(\Phi_{i, i+1} \Phi_{i+1, i}-\Phi_{i, i-1} \Phi_{i-1, i}\right),
$$

where the index $i$ is defined modulo $N_{g}$. Notice here that to each torus-invariant divisor $D_{\alpha}=C\left(\Sigma_{\alpha}\right)$, with $\Sigma_{\alpha}$ a three-subspace of the orbifold $Y=L^{m, n, m}$, we can associate a set of the bifundamental fields $\Phi_{i j}$. Geometrically, the relaton is that $\left\{\Phi_{i j}=0\right\}$ is the divisor $D_{\alpha}$ in the mesonic moduli space, which contains $X_{m, n}$. These fields have multiplicities $n_{\alpha}=\left|\left(v_{\alpha-1}, v_{\alpha}, v_{\alpha+1}\right)\right|$ [48], giving here $n_{1}=n_{4}=n$ and $n_{2}=n_{3}=m-$ see Table 2 ,

After adding a mass deformation of the form $\sum_{i=2 m+1}^{m+n} m_{i} \Phi_{i, i}^{2} / 2$ and integrating out the massive fields, we obtain

$$
\begin{aligned}
W_{m L^{m, n, m}}= & \sum_{i=1}^{2 m}(-)^{i} \Phi_{i, i-1} \Phi_{i-1, i} \Phi_{i, i+1} \Phi_{i+1, i} \\
& -\sum_{i=2 m+1}^{n-m} \lambda_{i}\left(\Phi_{i, i+1} \Phi_{i+1, i}-\Phi_{i, i-1} \Phi_{i-1, i}\right)^{2}
\end{aligned}
$$

with $n-m$ complex coupling constants $\lambda_{i}=1 / 2 m_{i}$. The corresponding F-term equations give rise to

$$
\begin{array}{ll}
v=\Phi_{i, i+1} \Phi_{i+1, i}=0 & \text { for all odd } i<2 m \\
u=\Phi_{j, j+1} \Phi_{j+1, j} & \text { for all } j \neq i,
\end{array}
$$

where again we focus on the branch of the moduli space which does not correspond to moving fractional branes along the residual singularity. In addition, we find the 


\begin{tabular}{|c|c|c|c|}
\hline 3-subspace & Fields & $Q_{B}$ & Multiplicity \\
\hline \hline$\Sigma_{1}$ & $\Phi_{j+1, j}$ & $-m$ & $n$ \\
\hline$\Sigma_{2}$ & $\Phi_{i, i+1}$ & $n$ & $m$ \\
\hline$\Sigma_{3}$ & $\Phi_{i+1, i}$ & $-n$ & $m$ \\
\hline$\Sigma_{4}$ & $\Phi_{j, j+1}$ & $m$ & $n$ \\
\hline
\end{tabular}

Table 2: Divisors, fields, charges, and multiplicities for the $L^{m, n, m}$ theories. Here, as in (4.50), the index $i$ is odd and smaller than $2 m$, while the index $j$ covers the remainder.

following gauge-invariant monomials

$$
w=\Phi_{12} \cdots \Phi_{m+n, 1}, \quad z=\Phi_{1, m+n} \cdots \Phi_{21}
$$

which then satisfy $u^{n} v^{m}=w z$. As an illustration, consider the $L^{1,3,1}$ theory. The F-terms lead to

$$
u=\Phi_{23} \Phi_{32}=\Phi_{34} \Phi_{43}=\Phi_{41} \Phi_{14}, \quad v=\Phi_{12} \Phi_{21}=0
$$

and we can construct the $U(1)_{1,2,3}$-invariant monomials

$$
w=\Phi_{12} \Phi_{23} \Phi_{34} \Phi_{41}, \quad z=\Phi_{14} \Phi_{43} \Phi_{32} \Phi_{21},
$$

which satisfy $u^{3} v=w z$.

In the general case the condition $v=0$ implies that either $w$ or $z$ vanishes. The moduli space is thus again two copies of $\mathbb{C}^{2}$, intersecting over $\mathbb{C}$.

We next perform $a$-maximization for the IR fixed point of the massive deformation. The requirement that the superpotential has R-charge 2 gives

$$
R\left[\Sigma_{1}\right]+R\left[\Sigma_{4}\right]=1, \quad R\left[\Sigma_{2}\right]+R\left[\Sigma_{3}\right]=1
$$

where the field-divisor map is given in Table2, The ABJ anomaly is then automatically satisfied. The trial central charge function is thus

$$
\begin{aligned}
a_{\text {trial }} & =\frac{27 N^{2}}{32} \sum_{i=1}^{N_{g}} R_{i, i+1} R_{i+1, i} \\
& =\frac{27 N^{2}}{32}\left(m R\left[\Sigma_{2}\right] R\left[\Sigma_{3}\right]+n R\left[\Sigma_{1}\right] R\left[\Sigma_{4}\right]\right),
\end{aligned}
$$

which is locally maximized when all R-charges are equal to $1 / 2$, at which point the central charge is

$$
\frac{a_{\mathcal{N}=4}}{a_{m L^{m, n, m}}}=\frac{32}{27 N_{g}}=\frac{32}{27(m+n)} .
$$


In accord with the $a$-theorem, the central charge of the infrared theory is strictly smaller than the central charge of the original theory given in [48], for all values of $m$ and $n$ :

$$
a_{L^{m, n, m}}=\frac{27 N^{2}}{16} m^{2} n^{2}\left[(2 m-n)(2 n-m)(m+n)+2\left(m^{2}-m n+n^{2}\right)^{3 / 2}\right]^{-1}
$$

Finally, we turn to the dual $Z$-minimization problem. Again, the homogeneity condition for $\Omega_{-}$leads to $\xi_{1}=3$ and $\xi_{3}=3 / 2$, the latter condition again coming from the expectation that the one-form part of $\Omega_{-}$is proportional to $\mathrm{d} \bar{v}^{2}$, precisely as for the Pilch-Warner solution and our discussion of the SPP theory. The $Z$ function is then

$$
Z=\frac{4 N_{g}}{3 \xi_{2}\left(3 N_{g}-2 \xi_{2}\right)},
$$

which is minimized for $\xi_{2}=3 N_{g} / 4$. The volume of $L^{m, n, m}$ after mass deformation and the volumes of the subspaces $\Sigma_{\alpha}$ are

$$
\operatorname{Vol}\left(m L^{m, n, m}\right)=\frac{32 \pi^{3}}{27 N_{g}}, \quad \operatorname{Vol}\left(\Sigma_{\alpha}\right)=\frac{16 \pi^{2}}{9 N_{g}} .
$$

Using again (3.47), we can verify that the conjectured relation between $Z$ and $a_{\text {trial }}$ indeed holds.

\section{Conclusion and outlook}

In this paper we have developed a deeper understanding of general supersymmetric $A d S_{5}$ solutions of type IIB supergravity using generalized geometry. Following on from [9], we have studied a pair of compatible pure spinors on a generalized Calabi-Yau cone, which generalizes the notion of a Kähler cone with zero first Chern class. The key point is that the complex structure on the cone is replaced by a generalized complex structure, which becomes an ordinary complex structure on a special type-change locus. Physically, this locus is the Abelian mesonic moduli space of the dual field theory. In section 2 we have introduced the notion of generalized Sasakian geometry, which shares many properties with Sasakian geometry. In particular, there is an underlying contact structure, and the associated Reeb vector field, dual to the R-symmetry, is generalized holomorphic, generalized Killing, and related to $r \partial_{r}$ via the integrable generalized complex structure $\mathcal{J}_{-}$. Away from the type-change locus, the transverse space to the Reeb foliation, rather than being Kähler as in the Sasakian case, is endowed with a triple of orthogonal symplectic forms satisfying a system of differential equations. 
Equipped with this definition of generalized Sasakian geometry, we then proved in section 3 the analogous result to [8]: the action for the bosonic supergravity fields is equal, when restricted to a space of generalized Sasakian structures, to the underlying contact volume, and thus depends only on the Reeb vector field. This implies that the Reeb vector field of a supersymmetric $A d S_{5}$ solution is obtained by minimizing the contact volume over a space of the Reeb vector fields under which the pure spinor $\Omega_{-}$has charge three. Since at the critical point this contact volume is equal to the inverse central charge of the dual field theory [11], this is conjecturally the geometric counterpart of $a$-maximization in four-dimensional $\mathcal{N}=1$ superconformal field theories.

A number of important questions and problems arise from this work. Firstly, our current understanding of the deformation space of generalized Sasakian structures is very limited. This is simply because this is a new structure, which we have only had chance to develop quite superficially in the course of a single paper; on the other hand, Sasakian geometry has been studied since 1960. Having said that, there is still no general understanding of the deformation space even for Sasakian manifolds, the only complete description being that for toric Sasakian manifolds given in [12]. We believe that a similar level of understanding should be achievable for generalized toric Sasakian manifolds, but leave this for future work. Notice that, in any case, our definition of generalized Sasakian geometry reduces to that of Sasakian geometry when the generalized complex structure is complex, and that the beta deformation of a Kähler cone is a cone over a generalized Sasakian manifold.

Secondly, we have not investigated the type-change locus $\mathscr{T}$ in any detail here. It is an important problem to understand what the constraints are on the type-change locus, and to classify the types of boundary conditions associated with the structures introduced in section 2 . We note that this is very much an open problem in generalized geometry, for which there are currently only some very preliminary results [33, 49].

In section 4 we bypassed most of the above open issues by focusing on some examples for which we have a fairly good understanding of the physics on the SCFT side of the correspondence. This allowed us to predict the existence of supersymmetric $A d S_{5}$ solutions of type IIB supergravity, with the same topology and toric symplectic structure as the cones $C\left(L^{m, n, m}\right)$. Although we do not know the pure spinor $\Omega_{-}$, we made some reasonable assumptions about the generalized geometry based on the dual field theories and on the Pilch-Warner solution that these solutions generalize, and thereby determined the type-change locus to be $\mathscr{T}=\mathbb{C}^{2} \cup_{\mathbb{C}} \mathbb{C}^{2}$. We were then able to compute the critical Reeb vector field and hence the contact volumes. Using the formulae in [11], 
we found perfect agreement with the central charges and R-charges of chiral primary fields computed via $a$-maximization in the dual SCFTs obtained by mass deformation. Perhaps the main issue raised here is why the toric symplectic structure is preserved after the renormalization group flow triggered by the mass deformation. In fact, the field theory interpretation of the contact or symplectic structure, which exists whenever the solution has non-zero D3-brane charge, is still unclear.

We hope to return to many of these issues in future work.

\section{Acknowledgements}

We thank Jerome Gauntlett, Dario Martelli and Daniel Waldram for discussions at an early stage of this project, and Davide Cassani and Luca Martucci for electronic communications. M. G. is supported by the Berrow Foundation and the Swiss National Fund, and J. F. S. by a Royal Society University Research Fellowship.

\section{A The hazards of dimensional reduction}

In section 3.1 we derived the equations of motion on $Y$ for the bosonic fields of type IIB supergravity from ten-dimensional equations, and then constructed an action $Z$ whose variation led to those equations. An alternative strategy to obtain $Z$ would have been to dimensionally reduce the type IIB action on $A d S_{5} \times Y$, where the "reduction" is along the $A d S_{5}$ direction. However, this approach is complicated by ambiguities associated with the self-duality of $F_{5}$, and to the lack of proper normalization of the Chern-Simons term [50]. In this appendix we outline the relation between these two approaches.

Even though the field equations of type IIB supergravity cannot be derived directly from the variation of an action, one can have recourse to a pseudo-action that leads to equations of motion that match the type IIB equations only when supplemented by the self-duality condition $\star F_{5}=F_{5}$. With $\tilde{F}_{5} \equiv F_{5}+\frac{1}{2} \mathrm{~d}\left(B \wedge C_{2}\right)$, this pseudo-action reads [51]

$$
\begin{aligned}
S_{\mathrm{IIB}}^{10}= & \frac{1}{2 \kappa_{10}^{2}} \int \mathrm{d}^{10} x \sqrt{-g_{\mathrm{E}}}\left(R_{\mathrm{E}}-2|P|^{2}-\frac{1}{2}|G|^{2}-\frac{1}{4}\left|\tilde{F}_{5}\right|^{2}\right) \\
& -\frac{1}{4 \kappa_{10}^{2}} \int \mathrm{d} C_{4} \wedge H \wedge C_{2} .
\end{aligned}
$$


After a Weyl rescaling $\bar{g}=g_{A d S}+g_{Y}=\mathrm{e}^{-2 \Delta} g_{\mathrm{E}}$ this becomes

$$
\begin{aligned}
S_{\mathrm{IIB}}^{10}= & \frac{1}{2 \kappa_{10}^{2}} \int_{A d S_{5} \times Y} \mathrm{~d}^{10} x \sqrt{\bar{g}} \mathrm{e}^{8 \Delta}\left(\bar{R}-18 \mathrm{e}^{-8 \Delta} \nabla_{M}\left(\mathrm{e}^{8 \Delta} \partial^{M} \Delta\right)+72|\mathrm{~d} \Delta|^{2}\right. \\
- & \left.-2|P|^{2}-\frac{1}{2} \mathrm{e}^{-4 \Delta}|G|^{2}-\frac{1}{4} \mathrm{e}^{-8 \Delta}\left|\tilde{F}_{5}\right|^{2}\right) \\
& -\frac{1}{4 \kappa_{10}^{2}} \int \mathrm{d} C_{4} \wedge H \wedge C_{2} .
\end{aligned}
$$

Splitting the integral into a part over $A d S_{5}$ and a part over $Y$, one finds

$$
\begin{gathered}
S_{\mathrm{IIB}}^{10}=\frac{\operatorname{Vol}\left(A d S_{5}\right)}{2 \kappa_{10}^{2}}\left[\int_{Y} \mathrm{~d}^{5} y \sqrt{g_{Y}} \mathrm{e}^{8 \Delta}\left(R\left(g_{Y}\right)-20+72|\mathrm{~d} \Delta|^{2}-2|P|^{2}-\frac{1}{2} \mathrm{e}^{-4 \Delta}|G|^{2}\right)\right. \\
\left.-\frac{1}{2} \int_{Y}\left(\mathrm{~d}^{5} y \sqrt{g_{Y}} \frac{\left|\tilde{F}_{5}\right|^{2}}{2}+f_{5} H \wedge C_{2}\right)\right] .
\end{gathered}
$$

Note that the prefactor $\operatorname{Vol}\left(A d S_{5}\right)$ here is infinite. Pragmatically, one can simply discard this prefactor in order to obtain an action for the bosonic fields on $Y$, although in a more systematic treatment this should be regularized holographically following Henningson and Skenderis [52]. The term $\left|\tilde{F}_{5}\right|^{2}$ should be understood only symbolically, since the diabolic self-duality property makes it vanish. Naively, one might be tempted to formally set $\left|\tilde{F}_{5}\right|^{2}=f_{5}^{2}$. Comparing with the action $Z$ in (3.18), we conclude that instead a factor of -2 is missing in front of the second line of (A.3). A similar factor was already pointed out by Belov and Moore [50].

\section{B The contact volume functional}

Is this appendix we consider the contact volume

$$
\operatorname{Vol}_{\sigma}(Y) \equiv \int_{Y} \sigma \wedge \frac{\omega_{T}^{2}}{2 !}=\frac{1}{8} \int_{Y} \sigma \wedge \mathrm{d} \sigma \wedge \mathrm{d} \sigma=\int_{Y} \operatorname{vol}_{\sigma} .
$$

as a functional on an appropriate space of contact structures on a fixed five-manifold $Y$. Thus here $\sigma$ is a contact one-form on $Y$. We begin by showing that this volume depends only on the unique Reeb vector field $\xi$ that is associated with $\sigma$. As we explain, this is analogous to the statement in symplectic geometry that the symplectic volume depends only on the cohomology class of the symplectic form. We then compute the first and second derivatives of the contact volume. In particular, provided one considers only deformations of the Reeb vector field that preserve $\sigma$, then the volume functional is strictly convex. These results generalize those of [8], 47] for Sasakian manifolds to 
general contact manifolds. Of course, the results that follow hold in arbitrary odd dimension, with appropriate replacements of dimension-dependent constants.

Consider a fixed contact one-form $\sigma$ on $Y$, and a one-parameter family of deformations $\sigma_{t}$, with $\sigma_{0}=\sigma$ and $t \in(-\epsilon, \epsilon) \subset \mathbb{R}$. We Taylor-expand $\sigma_{t}=\sigma+t \sigma^{\prime}+\mathcal{O}\left(t^{2}\right)$, with a similar expansion of the Reeb vector field $\xi_{t}=\xi+t \xi^{\prime}+\mathcal{O}\left(t^{2}\right)$. Since by definition $\left.\sigma_{t}\left(\xi_{t}\right)=1, \xi_{t}\right\lrcorner \mathrm{d} \sigma_{t}=0$, one immediately deduces the first order equations

$$
\left.\left.\sigma^{\prime}(\xi)=-\sigma\left(\xi^{\prime}\right), \quad \xi\right\lrcorner \mathrm{d} \sigma^{\prime}=-\xi^{\prime}\right\lrcorner \mathrm{d} \sigma
$$

We now compute

$$
\begin{aligned}
\int_{Y} \operatorname{vol}_{\sigma_{t}}-\int_{Y} \operatorname{vol}_{\sigma} & =\frac{t}{8}\left[\int_{Y} \sigma^{\prime} \wedge(\mathrm{d} \sigma)^{2}+2 \int_{Y} \sigma \wedge \mathrm{d} \sigma \wedge \mathrm{d} \sigma^{\prime}\right]+\mathcal{O}\left(t^{2}\right) \\
& =\frac{3 t}{8} \int_{Y} \sigma^{\prime} \wedge(\mathrm{d} \sigma)^{2}+\mathcal{O}\left(t^{2}\right) \\
& =-\frac{3 t}{8} \int_{Y} \sigma\left(\xi^{\prime}\right) \sigma \wedge(\mathrm{d} \sigma)^{2}+\mathcal{O}\left(t^{2}\right)
\end{aligned}
$$

where in going from the first to the second line we have integrated the second term by parts and used Stokes' theorem, and in going from the second to the third line we have used the first equation in (B.2). In particular, if we consider deformations of the contact structure that leave fixed the Reeb vector field, then by definition $\xi^{\prime}=0$ and the contact volume is invariant. Thus we may regard the contact volume as a functional of $\xi$, as opposed to $\sigma$, and we have then shown that the first derivative of the contact volume is

$$
\mathrm{dVol}_{\sigma}\left[\xi^{\prime}\right]=-3 \int_{Y} \sigma\left(\xi^{\prime}\right) \operatorname{vol}_{\sigma}
$$

Of course, this result reproduces that in [8], but here we have used only contact geometry. In the special case in which $\xi$ generates a $U(1)$ action on $Y$, the quotient $Y / U(1)$ is a symplectic orbifold and the contact volume is (proportional to) the symplectic volume of $Y / U(1)$. Deformations of the contact structure that leave $\xi$ invariant are then deformations of the symplectic structure on $Y / U(1)$ that leave the cohomology class fixed, which thus preserve the volume. More generally, such deformations leave fixed the basic cohomology class of the symplectic structure on the leaf space of the Reeb foliation.

We next deform again the contact form and the Reeb vector field as $\sigma_{t}=\sigma+t \sigma^{\prime \prime}+$ 
$\mathcal{O}\left(t^{2}\right)$ and $\xi_{t}=\xi+t \xi^{\prime \prime}+\mathcal{O}\left(t^{2}\right)$, and similarly compute

$$
\begin{aligned}
\left.\frac{\mathrm{d}}{\mathrm{d} t} \int_{Y} \sigma_{t}\left(\xi^{\prime}\right) \sigma_{t} \wedge\left(\mathrm{d} \sigma_{t}\right)^{2}\right|_{t=0}= & 8 \int_{Y} \sigma^{\prime \prime}\left(\xi^{\prime}\right) \operatorname{vol}_{\sigma}+\int_{Y} \sigma\left(\xi^{\prime}\right) \sigma^{\prime \prime} \wedge(\mathrm{d} \sigma)^{2} \\
& +2 \int_{Y} \sigma\left(\xi^{\prime}\right) \sigma \wedge \mathrm{d} \sigma \wedge \mathrm{d} \sigma^{\prime \prime} \\
= & -24 \int_{Y} \sigma\left(\xi^{\prime}\right) \sigma\left(\xi^{\prime \prime}\right) \operatorname{vol}_{\sigma} \\
& +8 \int_{Y} \sigma^{\prime \prime}\left(\xi^{\prime}\right) \operatorname{vol}_{\sigma}-2 \int_{Y} \mathrm{~d}\left(\sigma\left(\xi^{\prime}\right)\right) \wedge \sigma^{\prime \prime} \wedge \sigma \wedge \mathrm{d} \sigma .
\end{aligned}
$$

Here we have used precisely the same steps as when computing the first derivative in (B.3). To deal with the last line, we write

$$
\left.\mathrm{d}\left(\sigma\left(\xi^{\prime}\right)\right)=\mathcal{L}_{\xi^{\prime}} \sigma-\xi^{\prime}\right\lrcorner \mathrm{d} \sigma
$$

using Cartan's formula. We now also impose that our original deformation vector field $\xi^{\prime}$ preserves the initial contact one-form, so $\mathcal{L}_{\xi^{\prime}} \sigma=0$. This means that $\xi^{\prime}$ is in the Lie algebra of strict contact deformations of $\sigma$. Notice that a similar assumption was also made in [8], where the space of Sasakian metrics considered had a fixed isometry group, with the Reeb vector field varied in the Lie algebra of this group. Focusing on the last line in $(\mathrm{B} .5)$, we then have

$$
\begin{aligned}
8 \int_{Y} \sigma^{\prime \prime}\left(\xi^{\prime}\right) \operatorname{vol}_{\sigma}-2 \int_{Y} \mathrm{~d}\left(\sigma\left(\xi^{\prime}\right)\right) \wedge \sigma^{\prime \prime} \wedge \sigma \wedge \mathrm{d} \sigma & \left.=\int_{Y} \sigma \wedge \xi^{\prime}\right\lrcorner\left[(\mathrm{d} \sigma)^{2} \wedge \sigma^{\prime \prime}\right] \\
& =\int_{Y} \sigma\left(\xi^{\prime}\right) \sigma^{\prime \prime} \wedge(\mathrm{d} \sigma)^{2} \\
& =-8 \int_{Y} \sigma\left(\xi^{\prime}\right) \sigma\left(\xi^{\prime \prime}\right) \operatorname{vol}_{\sigma} .
\end{aligned}
$$

Altogether we have thus shown that the second derivative of the contact volume is

$$
\mathrm{d}^{2} \operatorname{Vol}_{\sigma}\left[\xi^{\prime}, \xi^{\prime \prime}\right]=12 \int_{Y} \sigma\left(\xi^{\prime}\right) \sigma\left(\xi^{\prime \prime}\right) \operatorname{vol}_{\sigma},
$$

thus showing that $\operatorname{Vol}_{\sigma}(Y)$ is strictly convex. Again, notice that this formula reproduces that in [8].

\section{References}

[1] J. M. Maldacena, "The large N limit of superconformal field theories and supergravity," Adv. Theor. Math. Phys. 2 (1998) 231 [Int. J. Theor. Phys. 38 (1999) 1113] arXiv:hep-th/9711200. 
[2] I. R. Klebanov and E. Witten, "Superconformal field theory on threebranes at a Calabi-Yau singularity," Nucl. Phys. B 536 (1998) 199 [arXiv:hep-th/9807080].

[3] J. M. Figueroa-O'Farrill, "Near-horizon geometries of supersymmetric branes", arXiv:hep-th/9807149.

[4] B. S. Acharya, J. M. Figueroa-O'Farrill, C. M. Hull and B. Spence, "Branes at conical singularities and holography," Adv. Theor. Math. Phys. 2 (1999) 1249 arXiv:hep-th/9808014.

[5] D. R. Morrison and M. R. Plesser, "Non-spherical horizons. I," Adv. Theor. Math. Phys. 3 (1999) 1 arXiv:hep-th/9810201].

[6] D. Anselmi, J. Erlich, D. Z. Freedman and A. A. Johansen, "Positivity constraints on anomalies in supersymmetric gauge theories," Phys. Rev. D 57, 7570 (1998) arXiv:hep-th/9711035.

[7] S. S. Gubser, "Einstein manifolds and conformal field theories," Phys. Rev. D 59, 025006 (1999), [arXiv: hep-th/9807164].

[8] D. Martelli, J. Sparks and S. T. Yau, "Sasaki-Einstein manifolds and volume minimisation," Commun. Math. Phys. 280, 611 (2008) arXiv:hep-th/0603021.

[9] M. Gabella, J. P. Gauntlett, E. Palti, J. Sparks and D. Waldram, "Ad $S_{5}$ Solutions of Type IIB Supergravity and Generalized Complex Geometry," Commun. Math. Phys. 299 (2010) 365 arXiv:0906.4109 [hep-th]].

[10] N. Hitchin, "Generalized Calabi-Yau manifolds," Quart. J. Math. Oxford Ser. 54 (2003) 281-308 arXiv:math/0209099.

[11] M. Gabella, J. P. Gauntlett, E. Palti, J. Sparks and D. Waldram, "The central charge of supersymmetric $A d S_{5}$ solutions of type IIB supergravity," Phys. Rev. Lett. 103 (2009) 051601 arXiv:0906.3686 [hep-th]].

[12] D. Martelli, J. Sparks and S. T. Yau, "The geometric dual of a-maximisation for toric Sasaki-Einstein manifolds," Commun. Math. Phys. 268, 39 (2006) arXiv:hep-th/0503183.

[13] J. Sparks, "Sasaki-Einstein Manifolds," arXiv:1004.2461 [math.DG]. 
[14] K. A. Intriligator and B. Wecht, "The exact superconformal R-symmetry maximizes a," Nucl. Phys. B 667, 183 (2003), arXiv:hep-th/0304128.

[15] A. Butti and A. Zaffaroni, "R-charges from toric diagrams and the equivalence of a-maximization and Z-minimization," JHEP 0511, 019 (2005) arXiv:hep-th/0506232.

[16] R. Eager, "Equivalence of A-Maximization and Volume Minimization," arXiv: 1011.1809 [hep-th].

[17] H. Geiges, "Symplectic couples on 4-manifolds," Duke Math. J. 85 (1996), 701711.

[18] L. Martucci, "D-branes on general $N=1$ backgrounds: Superpotentials and Dterms," JHEP 0606, 033 (2006) arXiv:hep-th/0602129.

[19] R. Minasian, M. Petrini and A. Zaffaroni, "Gravity duals to deformed SYM theories and generalized complex geometry," JHEP 0612, 055 (2006) arXiv:hep-th/0606257].

[20] M. Gualtieri, "Generalized complex geometry," Oxford University DPhil thesis, arXiv:math/0401221 [math-DG].

[21] O. Lunin and J. M. Maldacena, "Deforming field theories with U(1) x U(1) global symmetry and their gravity duals," JHEP 0505, 033 (2005) arXiv:hep-th/0502086].

[22] A. Butti, D. Forcella, L. Martucci, R. Minasian, M. Petrini and A. Zaffaroni, "On the geometry and the moduli space of beta-deformed quiver gauge theories," JHEP 0807, 053 (2008) arXiv:0712.1215 [hep-th]].

[23] M. Cvetic, H. Lu, D. N. Page and C. N. Pope, "New Einstein-Sasaki spaces in five and higher dimensions," Phys. Rev. Lett. 95 (2005) 071101 arXiv:hep-th/0504225].

[24] D. Martelli and J. Sparks, "Toric Sasaki-Einstein metrics on $S^{2} \times S^{3}$," Phys. Lett. B 621 (2005) 208 [arXiv:hep-th/0505027].

[25] M. Grana, R. Minasian, M. Petrini and A. Tomasiello, "Supersymmetric backgrounds from generalized Calabi-Yau manifolds," JHEP 0408, 046 (2004) [arXiv: hep-th/0406137]. 
[26] M. Grana, R. Minasian, M. Petrini and A. Tomasiello, "Generalized structures of $N=1$ vacua," JHEP 0511, 020 (2005) arXiv:hep-th/0505212.

[27] P. Koerber, "Lectures on Generalized Complex Geometry for Physicists," arXiv: 1006.1536 [hep-th].

[28] M. Wijnholt, "Parameter Space of Quiver Gauge Theories," arXiv:hep-th/ 0512122.

[29] S. J. . Gates, C. M. Hull and M. Rocek, "Twisted Multiplets And New Supersymmetric Nonlinear Sigma Models," Nucl. Phys. B 248, 157 (1984).

[30] A. Kapustin and Y. Li, "Topological sigma-models with H-flux and twisted generalized complex manifolds," arXiv:hep-th/0407249.

[31] N. Halmagyi and A. Tomasiello, "Generalized Kaehler Potentials from Supergravity," Commun. Math. Phys. 291 (2009) 1 [arXiv:0708.1032 [hep-th]].

[32] A. Tomasiello, "Reformulating Supersymmetry with a Generalized Dolbeault Operator," JHEP 0802, 010 (2008) [arXiv:0704.2613 [hep-th]].

[33] H. Bursztyn, G. Cavalcanti and M. Gualtieri "Reduction of Courant algebroids and generalized complex structures", Adv. Math. 211, 726 (2007) [arXiv: math/0509640v3 [math.DG]].

[34] J. H. Schwarz, "Covariant Field Equations Of Chiral N=2 D=10 Supergravity," Nucl. Phys. B 226 (1983) 269.

[35] J. P. Gauntlett, D. Martelli, J. Sparks and D. Waldram, "Supersymmetric AdS(5) solutions of type IIB supergravity," Class. Quant. Grav. 23, 4693 (2006) arXiv:hep-th/0510125.

[36] D. Lust, F. Marchesano, L. Martucci and D. Tsimpis, "Generalized nonsupersymmetric flux vacua," JHEP 0811 (2008) 021 [arXiv:0807.4540 [hep-th]].

[37] E. Lerman, "Contact toric manifolds," J. Symplectic Geom. 1 (2003), no. 4, 785828.

[38] D. Anselmi, D. Z. Freedman, M. T. Grisaru and A. A. Johansen, "Nonperturbative formulas for central functions of supersymmetric gauge theories," Nucl. Phys. B 526 (1998) 543 arXiv:hep-th/9708042. 
[39] D. Berenstein, C. P. Herzog, I. R. Klebanov, "Baryon spectra and AdS /CFT correspondence," JHEP 0206 (2002) 047. hep-th/0202150].

[40] K. A. Intriligator, B. Wecht, "Baryon charges in 4-D superconformal field theories and their AdS duals," Commun. Math. Phys. 245 (2004) 407-424. [hep-th/0305046].

[41] C. P. Herzog, J. McKernan, "Dibaryon spectroscopy," JHEP 0308 (2003) 054. hep-th/0305048].

[42] C. P. Herzog, J. Walcher, "Dibaryons from exceptional collections," JHEP 0309 (2003) 060. hep-th/0306298].

[43] R. G. Leigh and M. J. Strassler, "Exactly Marginal Operators And Duality In Four-Dimensional N=1 Supersymmetric Gauge Theory," Nucl. Phys. B 447, 95 (1995) arXiv:hep-th/9503121.

[44] K. Pilch and N. P. Warner, "A new supersymmetric compactification of chiral IIB supergravity," Phys. Lett. B 487, 22 (2000) arXiv:hep-th/0002192.

[45] A. Khavaev, K. Pilch and N. P. Warner, "New vacua of gauged N $=8$ supergravity in five dimensions," Phys. Lett. B 487 (2000) 14 arXiv:hep-th/9812035].

[46] A. M. Uranga, "Brane Configurations for Branes at Conifolds," JHEP 9901 (1999) 022 arXiv:hep-th/9811004].

[47] A. Futaki, H. Ono, G. Wang, "Transverse Kähler geometry of Sasaki manifolds and toric Sasaki-Einstein manifolds," J. Diff. Geom. 83 (2009), 585-636.

[48] S. Franco, A. Hanany, D. Martelli, J. Sparks, D. Vegh and B. Wecht, "Gauge theories from toric geometry and brane tilings," JHEP 0601 (2006) 128 arXiv:hep-th/0505211.

[49] H. Bursztyn, G. Cavalcanti and M. Gualtieri "Generalized Kahler and hyperKahler quotients", arXiv:math/0702104v1 [math.DG].

[50] D. Belov and G. W. Moore, "Conformal blocks for $\mathrm{AdS}(5)$ singletons," arXiv:hep-th/0412167.

[51] J. Polchinski, "String theory. Vol. 2: Superstring theory and beyond," Cambridge, UK: Univ. Pr. (1998). 
[52] M. Henningson and K. Skenderis, "The holographic Weyl anomaly," JHEP 9807, 023 (1998) arXiv:hep-th/9806087. 